FINAL TECHNICAL REPORT

Award Number DE-FG03-00SF22169

\title{
DEVELOPMENT OF DESIGN CRITERIA FOR FLUID INDUCED STRUCTURAL VIBRATIONS IN STEAM GENERATORS AND HEAT EXCHANGERS
}

\author{
$\underline{\text { Principal Investigators }}$ \\ Ivan Catton, Professor of Engineering \\ and \\ Vijay K. Dhir, Professor of Engineering \\ $\underline{\text { Team Members }}$ \\ Deepanjan Mitra \\ Omar Alquaddoomi \\ Pierangelo Adinolfi \\ Department of Mechanical and Aerospace Engineering \\ School of Engineering and Applied Science \\ University of California, Los Angeles \\ Los Angeles, California 90095
}




\section{Table of Contents}

1. Introduction

1.1 Nature of Fluid-Elastic Instability $\quad$................................ 1

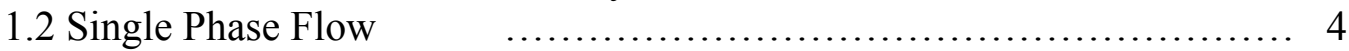

1.3 Two Phase Flow $\quad$........................................... 5

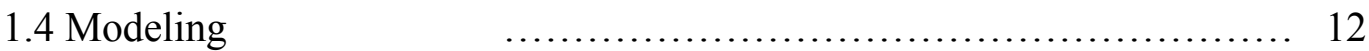

1.5 Objective of Present Study $\quad$.............................. 14

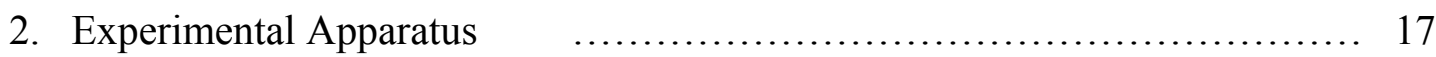

2.1 Single Phase Experiment $\quad$............................. 17

2.1.1 Flow Loop Description $\quad$.............................. 17

2.1.2 Test Section $\quad$.......................................... 19

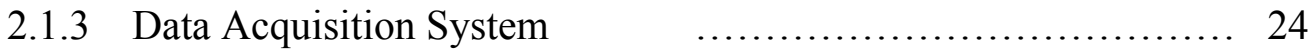

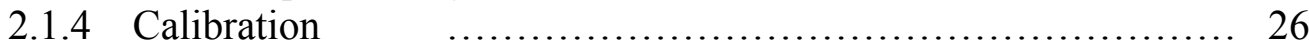

2.2 Two Phase Experiment $\quad$............................................... 29

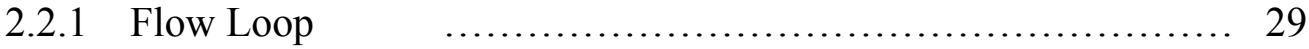

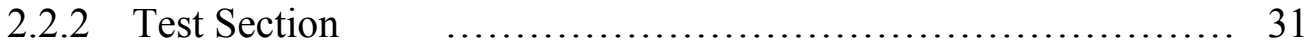

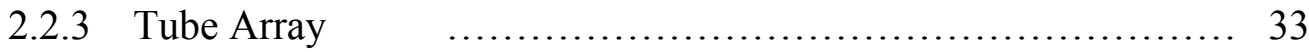

2.2.4 Measurement and Data Acquisition System $\quad$.................. 35

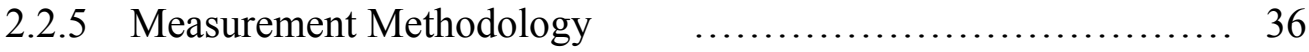

2.2.5.1 Flow Velocity Measurement ….......................... 36

2.2.5.2 Frequency Measurement $\quad$............................. 36

2.2.5.3 Damping Ratio Measurement ............................ 37

2.2.5.4 Tube Displacement $\quad$................................ 39

2.2.5.5 Void Fraction $\quad$.............................. 39

3. Fluid-elastic Instability Experiments $\quad$............................. 41

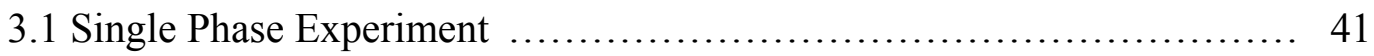

3.1.1 Data Processing Methods $\quad$................................ 41

3.1.1.1 Image Processing $\quad$............................. 41

3.1.1.2 Tube motion analysis: preliminary definitions $\quad$........... 43

3.1.1.3 Data Reduction: Interpolation Method $\quad$................. 44

3.1.1.4 Data Reduction: Correlation Method $\quad$................ 45

3.1.2 Results $\quad$............................................. 48

3.1.2.1 Normal Triangular Array $\quad$.............................. 49

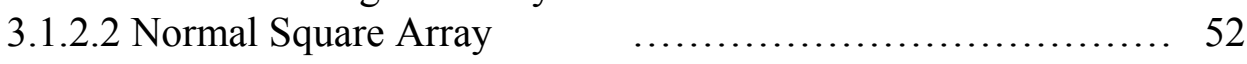

3.1.2.3 Vortex-Shedding and Frequency Response Observations $\quad$.. 54

3.1.2.4 Comparison: Array Configurations \& Other Sources $\quad \ldots \quad 56$ 
3.2.1 Preliminary Results $\quad$.................................. 60

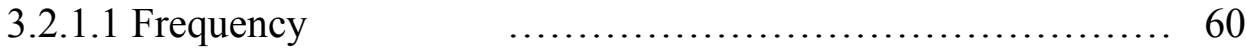

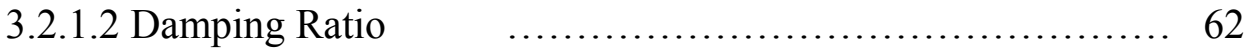

3.2.1.3 Void Fraction $\quad$................................... 64

3.2.1.4 Fluid-elastic Instability $\quad$............................ 67

3.2.2 Fluid-elastic Instability Experiments $\quad \ldots \ldots \ldots \ldots \ldots \ldots \ldots \ldots 68$

3.2.2.1 Air-water Flow $\quad$................................. 68

3.2.2.2 Fluid-elastic instability map for air-water two-phase flow $\quad \ldots \quad 73$

3.2.2.3 Experiments with a single flexible tube in a rigid array $\quad \ldots 74$

3.2.2.4 Steam-water tests and elevated temperature air-water tests $\ldots 75$

3.2.2.5 Air-water flow experiments at elevated temperatures $\quad \ldots 76$

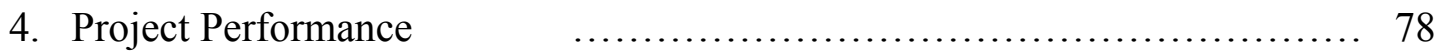

4.1 Single Phase Experiment $\quad$................................. 78

4.2 Two Phase Experiment $\quad$................................. 78

5. Project Publications, Presentations, and Technology Transfer $\quad \ldots \ldots \ldots \ldots . . .80$

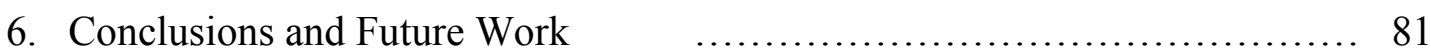

6.1 Single Phase Experiment $\quad$................................. 81

6.1.1 Conclusions $\quad$................................... 81

6.1.2 Future Work $\quad$.................................... 82

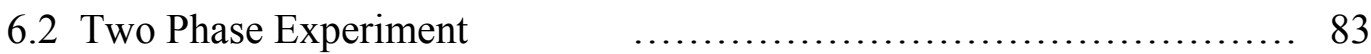

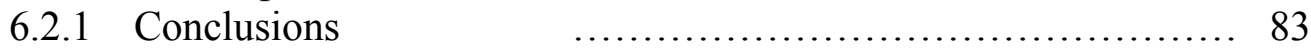

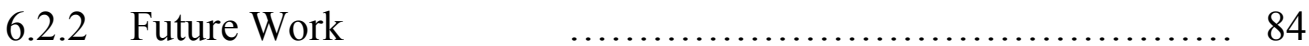

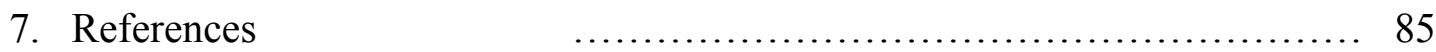




\section{Introduction}

Flow-induced vibration in heat exchangers has been a major cause of concern in the nuclear industry for several decades. Many incidents of failure of heat exchangers due to apparent flow-induced vibration have been reported through the USNRC incident reporting system. Almost all heat exchangers have to deal with this problem during their operation. The phenomenon has been studied since the 1970s and the database of experimental studies on flow-induced vibration is constantly updated with new findings and improved design criteria for heat exchangers. In the nuclear industry, steam generators are often affected by this problem. However, flow-induced vibration is not limited to nuclear power plants, but to any type of heat exchanger used in many industrial applications such as chemical processing, refrigeration and air conditioning. Specifically, shell and tube type heat exchangers experience flow-induced vibration due to the high velocity flow over the tube banks. Flow-induced vibration in these heat exchangers leads to equipment breakdown and hence expensive repair and process shutdown.

Flow-induced vibration, as the name suggests, is the vibration of a structure (in this case, heat exchanger tubes) due to the flow of a fluid around it. It is a fluid-structure interaction problem. When certain critical conditions are exceeded, an instability is induced in the system causing the tubes to vibrate with large amplitudes. The consequence of this is the damage of tubes in any of the three following ways: (1) For tubes rigidly attached to a supporting plate such as at the base, vibrations induce stress at the tube root causing a circumferential crack and hence snapping of the tube; (2) For tubes located near the mid span where the holes in the supporting plate are larger the tube to allow the flow of fluid through it, large amplitude vibrations cause the tubes to hit against the holes causing a groove to be formed at the support; (3) Finally in the absence of supporting plates, the tubes might hit each other when the amplitude of vibrations is large. In all the three cases, fretting wear of the tubes and hence damage will result in the long run.

Flow-induced vibration in structures is caused by three mechanisms. When a fluid flows over a bluff structure, vortices are shed behind it that cause an alteration of the flow field. This change in the flow field causes vibrations. This is known as vortex shedding instability. Another mechanism is galloping or flutter that induces vibrations in a noncircular or asymmetric body. Galloping in ice coated transmission wires is a common example. In aerodynamics, this mechanism is known as flutter as applied to airfoils. For flow over a tube bank or array, a third type of instability mechanism known as fluidelastic instability is the major cause of flow-induced vibration. Since fluid-elastic instability is the most important mechanism for heat exchangers and forms the basis of this research work, a description of this instability mechanism is provided in the next subsection.

\subsection{Nature of Fluid-Elastic Instability}

From a mechanistic view, the flow field around an array of flexible tubes causes the tube to be displaced from its initial position. This displacement causes a further shift in flow field changing the fluid forces acting on the tubes. The damping force of the tube 
that tries to restore it back to its equilibrium position opposes this change in fluid force. Thus, a competition results between the energy input by the fluid force and the energy expended in damping. When the energy expended in damping is more than the energy input by the fluid, the vibrations die down. However, if the fluid forces dominate, sufficient energy is imparted to the tube to sustain the vibrations and an unstable situation is reached where the tube vibrates with large amplitude. In shell-and-tube heat exchangers, this limit is known as fluid-elastic instability and leads to tube damage as described previously. A schematic of this mechanism is shown in Figure 1.1

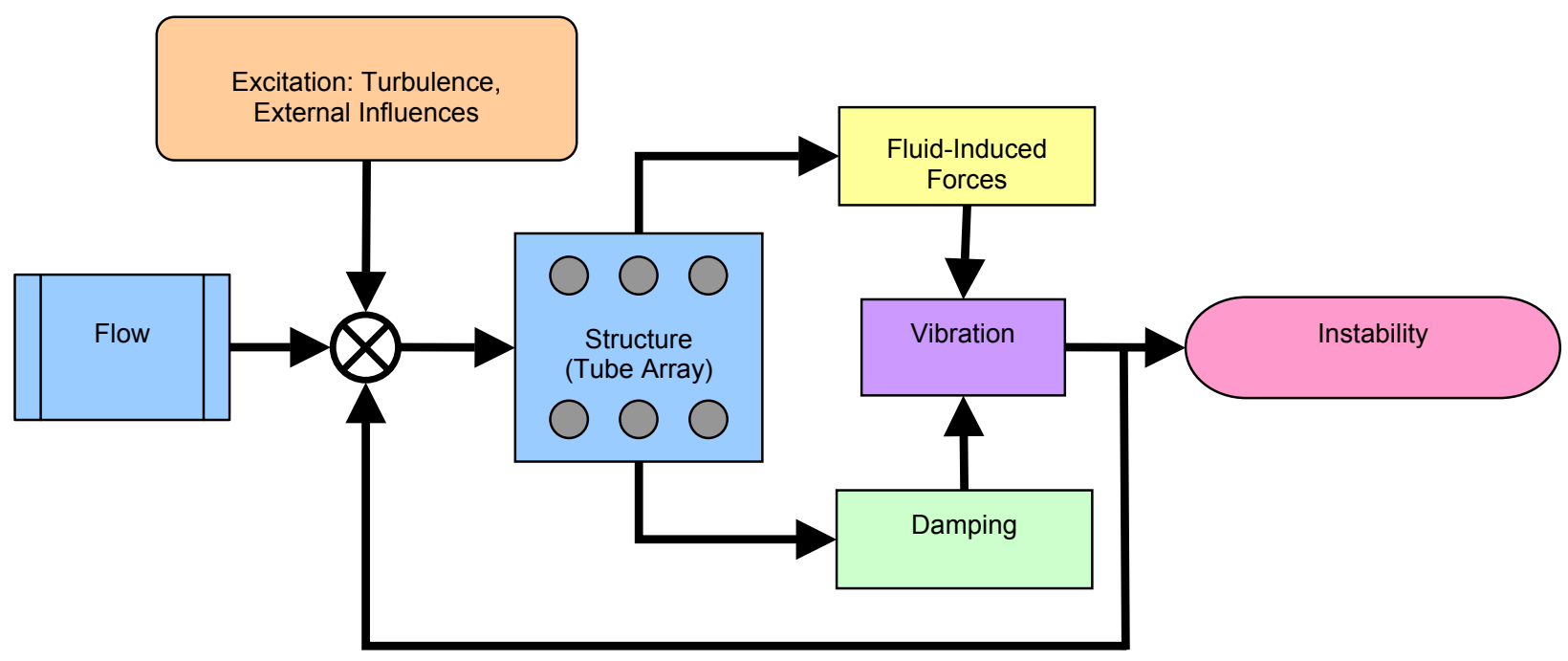

Figure 1.1: Mechanism of fluid-elastic instability in tube array

In heat exchangers, both cross flow regions where the fluid flows perpendicular to the tubes and axial flow regions where the fluid flows along the axis of the tubes exist. In addition, the fluid around the tubes consists of single phase and two-phase regions. Fluidelastic instability can occur for all the conditions described above.

One of the first mentions of this phenomenon in the literature was made by Roberts (1966) who observed the instability in a row of flexibly mounted tubes. He observed that the tubes vibrated with large amplitude beyond a critical velocity of the fluid flow. The instability was attributed to the time lag between the tube displacement and the fluid force. This was later verified by the experiments of Connors (1970) which were conducted in an array of tubes suspended by piano wires subjected to cross flow of water. He used tubes which were 8 inches long with a diameter of 1 inch. The experiments were conducted with a single tube, a row of tubes and a tube array. The vibrations were measured using strain gage transducers. The natural frequency of the tubes was either 10,40 or $200 \mathrm{~Hz}$. Connors attributed the instability to fluid forces induced by the displacement of the tubes and the interaction between them. He presented an instability map based on the dimensionless reduced velocity and the mass damping parameter, which is still commonly used for presenting results of fluid-elastic instability. 
Such a correlation is suggested by a dimensional analysis of the governing parameters and takes the form

$$
\frac{V_{c r}}{f_{n} D}=K\left(\frac{2 \pi m \zeta}{\rho D^{2}}\right)^{b}
$$

where $V_{c r}$ is the critical velocity of the fluid, $f_{n}$ is the natural frequency of the tube, $D$ is the outer diameter, $m$ is the mass per unit length of the tube, $\zeta$ is the damping ratio of the tube in vacuum, and $\rho$ is the fluid density. $K$ and $b$ are coefficients obtained by fitting experimental data. For his experiments, Connors obtained values of 9.9 and 0.5 for $K$ and $b$ respectively. The equation correlates the two non-dimensional groups -reduced velocity, $V_{c r} / f_{n} D$, and the mass damping parameter, $2 \pi m \zeta / \rho D^{2}$. The correlation of Eqn. 1.1 is often referred to as the Connors' criterion. The tube vibration was also observed visually leading to the conclusion that the tubes vibrated in synchronous oval orbits. This was later verified by Chen and Jendrzejczyk (1981, ref) who observed the motion of the tubes in an array in synchronous orbits. Graphical results of the orbital motion of the tubes are presented in their paper.

An alternate formulation for describing fluid-elastic instability used by some researchers has recently gained importance due to the article by Price (2001, ref). His analysis is based on a dimensional analysis of interstitial flow through cylinder arrays which yields the following non-dimensional groups

$$
f\left(\operatorname{Re}, T u, T / D, L / D, V_{c r} / f_{n} D, \delta, m / \rho D^{2}\right)
$$

where $R e$ is the Reynolds number of the flow, $T u$ is the freestream turbulence, $T$ and $L$ are the transverse and longitudinal separation between the cylinders. Drawing an analogy between flow across cylinder arrays and the classical analysis of binary flutter analysis of a 2 degree-of-freedom airfoil, the author suggests that the mass term and the damping term should act independently resulting in a correlation of the form

$$
\frac{V_{c r}}{f_{n} D}=K \delta^{a}\left(\frac{m}{\rho D^{2}}\right)^{b}
$$

The author also contradictorily mentions that, intuitively, since instability is caused due to a competition between the energy absorbed by the cylinder and the energy dissipated by it, damping should have a significant effect. Some experimental data however, seem to support the author's view that the exponent on the damping term is much less than 0.5 as obtained by Connors.

Nonetheless, the impact of Connors' work is so great that most of the research carried out to date use Connnors' criterion to correlate the experimental results of the instability.The next section reviews the literature in fluid-elastic instability subsequent to Connors' pioneering work. The review is limited to cross flow of fluid and is categorized as single or two-phase flow. Some recent findings and design recommendations available in literature are also reviewed. 


\subsection{Single-phase flow}

Since the results of Connors were made available and fluid-elastic instability was identified as the dominant component of flow-induced vibration in heat exchangers, a number of researchers took up the task of conducting systematic experiments for identifying the parameters causing fluid-elastic instability. Simultaneously, efforts were made in trying to develop models that could provide a theoretical basis for the mechanisms underlying fluid-elastic instability. Most of the early work was carried out with single-phase fluids such as air and water due to the simpler nature of the experiments. Even to date, the number of experiments involving single-phase fluids exceeds those of two-phase mixtures.

Tanaka and Takahara (1981) were among the earliest to conduct experiments on a $4 \times 7$ flexible array in water. The cylinders had a diameter of $0.03 \mathrm{~m}$ and a length of 0.3 $\mathrm{m}$. They used the alternate formulation of the form

$$
\frac{V_{r}}{f_{n} D}=K\left(\frac{m}{\rho D^{2}}\right)^{c}(2 \pi \zeta)^{d}
$$

to correlate their instability results. They did not see an isolated effect of the mass term in air. However, in water the effect was seen with the best fit being obtained for a value of 0.33 for $c$ and 0.2 for $d$ indicating a much lower effect of the damping term. They also studied the effects of detuning by changing the frequency of the test tube slightly as compared to the other tubes in the array. Their results indicated that for detuned tubes, the critical reduced velocity increased by $60 \%$ in air for some cases. Weaver and Patrick (1982) reviewed the experimental work carried out in single-phase fluid-elastic instability and suggested some design guidelines for heat exchangers based on the Connors' criterion. Values for $K$ and $b$ were suggested for different array geometries. For values of mass damping parameters below 0.3 , the reduced velocity was found to be constant, i.e. the mass damping parameter had no apparent effect. Above this value, the exponent $b$ varied between 0.3 and 0.48 and the value of $K$ between 2.5 and 4.8 for different array geometries. They pointed out the need for more experiments at very low and very high values of the mass damping parameters. A subsequent review by Pettigrew and Taylor (1991) presented a stability map based on almost 300 data points consolidated from different research papers. The authors pointed out the inconsistencies in reporting the instability data by different researchers. While compiling their database, they found that different researchers used different definitions for the reduced velocity and also reported damping ratios in different media. In presenting their own results, the authors used the natural frequency and damping in still water. Their instability map yielded a value of 3 for $K$ and 0.5 for $b$. The instability map is reproduced in Figure 1.2. The review of Pettigrew and Taylor also pointed out that some tests conducted on an all flexible tube array became unstable at lower reduced velocities as compared to those conducted on one flexible tube in a rigid array, thus stressing the necessity of conducting tests with fully flexible arrays. 


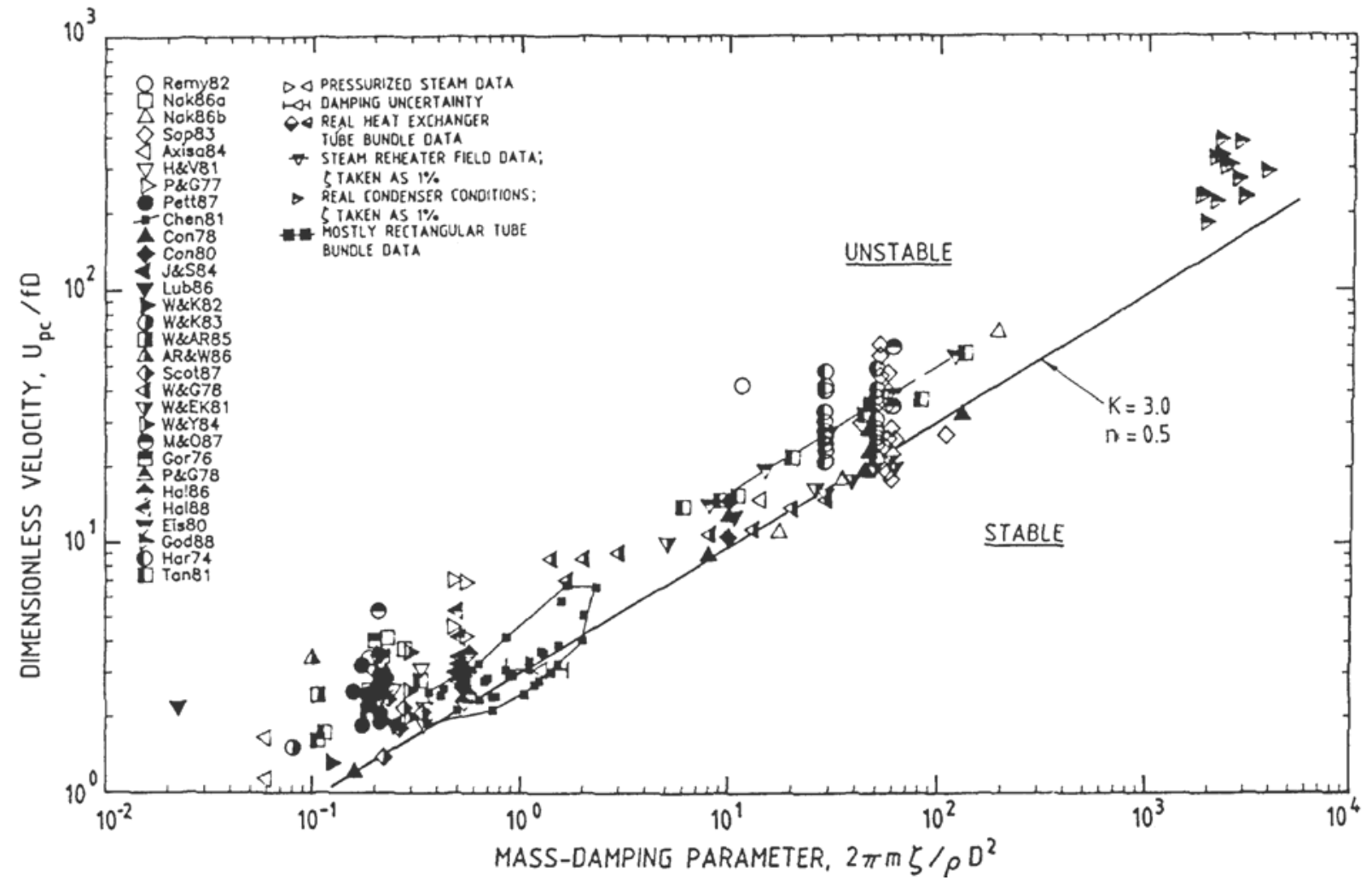

Figure 1.2: Single-phase fluid-elastic instability map (Reproduced from Pettigrew and Taylor, 1991)

A recent work on design recommendations for avoiding fluid-elastic instability in heat exchanger bundles by Schroder and Gelbe (1999) reveals a dependence of the factors $K$ and $b$ on the pitch to diameter ratio of the tube array and also the geometric arrangement of the array.

The main conclusion to be drawn from the work on single phase flow is that most of the instability results are modeled using Connors' criterion. Also, the need for a uniform standard for reporting instability results is apparent upon review of the literature.

\subsection{Two-phase flow}

As compared to single-phase flow cases, research on fluid-elastic instability in two-phase flow has been lacking. Experimental difficulties and uncertainties in measurement of experimental parameters are the main deterrents in such experiments. In addition, two-phase flow introduces two new parameters; namely the void fraction and the flow regime both of which affect the instability. Most of the work in two-phase flows has been carried out for air-water flows, the justification being that an air-water flow system is fundamentally no different than steam-water flow. Several researchers (Pettigrew et al., 1995, Feenstra et al., 1995, 2002) have reported some data obtained from tests carried out with liquid-vapor freon. Experiments with Freon are closer to steam-water and can be easily carried out due to the lower temperatures involved. However, very few test have been carried out for steam-water mixtures, which are most representative of flows in practical heat exchanger equipment. 
Heilker and Vincent (1981) conducted air-water flow tests with triangular and square arrays. Their tubes had diameters of $19 \mathrm{~mm}$ and $22 \mathrm{~mm}$ and a length of $0.91 \mathrm{~m}$. The tubes were supported with O-rings at each end in removable end plates. Strain gages and biaxial accelerometers were mounted on some test tubes to monitor the tube deflection and the natural frequency. The maximum water flow rate achievable in their loop was $0.32 \mathrm{~m}^{3} / \mathrm{s}$. Their experiments were conducted for void fractions higher than 0.72. Based on their experiments, they suggested a value of 5 for the instability constant, $K$. Remy (1982) conducted air-water flow experiments on a square array having a pitchto-diameter $(P / D)$ ratio of 1.44 . The tubes were $1 \mathrm{~m}$ long and had a diameter of $0.01 \mathrm{~m}$. The tubes vibrated with a natural frequency of $56.6 \mathrm{~Hz}$ in air and $49.7 \mathrm{~Hz}$ in water. The test tubes were instrumented with biaxial accelerometers contained within them to measure the vibration. The maximum flow rates achievable in the loop were $0.014 \mathrm{~m}^{3} / \mathrm{s}$ for water and $0.3 \mathrm{~m}^{3} / \mathrm{s}$ for air. Void fraction of the mixture was measured with an optical fiber probe. The author could not see a clear instability threshold in the two-phase flow experiments. For the single-phase cases, he adopted the approach of Tanaka et al. and grouped the damping term in the mass damping parameter with the instability constant $K$, in order to avoid damping issues. Remy did not recommend any values of the instability constant for two-phase flow cases, but suggested a value of 1.17 for the combined parameter $K \sqrt{2 \pi \zeta}$ in single-phase flow.

Pettigrew et al. $(1985,1989)$ reported on some comprehensive experimental work carried out with air-water flow for studying fluid-elastic instability pertaining to nuclear applications. They tested normal square, rotated square, normal triangular $\left(30^{\circ}\right)$, and parallel triangular $\left(60^{\circ}\right)$ arrays having a $P / D$ ratio of 1.47 except in the case of normal triangular array where additional tests with a $P / D$ ratio of 1.32 were also carried out. The tube array was cantilevered with the tubes vibrating at a natural frequency of $33 \mathrm{~Hz}$ in air. The stainless steel tubes were $0.6 \mathrm{~m}$ long and $0.013 \mathrm{~m}$ in diameter. The tube vibration was measured using pairs of strain gages installed near the clamped ends of selected test tubes. Their flow loop could supply water at $0.303 \mathrm{~m}^{3} / \mathrm{s}$ air at 960 standard cubic feet per minute $(\mathrm{scfm})$ through a rectangular test section measuring $0.114 \mathrm{~m} \times 0.6 \mathrm{~m}$. Tests were conducted with an all flexible tube array and a single flexible tube in a rigid array. During an experimental run, a constant void fraction was maintained by controlling the water and air flow rates. In this manner, void fractions from 5-99\% were systematically tested. The authors used the assumption of a homogenous mixture in the calculation of their parameters. Damping, which is difficult to measure during flow conditions was measured using a least squares fit to the amplitude spectrum of the vibration signal. A detailed discussion of this method is available in Pettigrew et al. (1985). The authors noted that since the spectral peak narrowed at the onset of instability giving the appearance of reduced damping, the damping measurements were carried out at a point halfway between the instability point. At lower void fractions of about 5\%, two dominant peaks were seen in the amplitude spectrum indicating hydrodynamic interaction between tubes, and possible periodic wake shedding. Their experimental results suggest a value of 4 for $K$. As reported for some single-phase flow cases, the critical reduced velocities were lower for an all flexible array as compared to a single flexible tube in an array. Pettigrew et al. also saw an effect of the $P / D$ ratio on the slope of the instability curve indicated by the value of the constant, $K$. This effect was more pronounced in two-phase flow cases and was attributed to hydrodynamic coupling enhanced by confinement. Another 
conclusion reached by the researchers was that in the intermittent flow regimes at void fractions higher than about $80 \%$, instability was achieved at lower values of critical velocity distorting the general trend of the instability curve, and thereby suggesting that operation of heat exchangers in such flow regimes is highly undesirable.

A recent paper by Pettigrew et al. (2001) investigated the effect of the geometry of the tube array on tube vibration. This work was carried out using the same loop used in their previous studies with an all flexible cantilevered array as well as a single flexible tube in a rigid array. They found an effect of both the pitch-to-diameter ratio and the array geometry on the instability constant $K$. The effect was most pronounced with the rotated triangular array. In continuation with this work, a recent paper by the authors (Pettigrew et al., 2002) presents a comparison of results of tests conducted in air-water, Freon 22 and Freon 134a cross-flow around a rotated triangular tube array. The damping values obtained are found to be similar in air-water and freon in spite of the different physical properties of the two fluids prompting the authors to point out the need for a better understanding of the principal parameters such as surface tension governing damping in two-phase flow. They also suggest studying the detailed characteristics of flow regimes between the tubes for the two fluid systems considered.

Axisa, Villard, et al. (1984) were one of the first researchers to conduct air-water and steam-water tests in their experimental test facility in France and present comparisons between the two systems. Their experiments were conducted on a $6 \times 10$ square array of Inconel tubes that were clamped at one end and nearly clamped at the other allowing for minor adjustments in the natural frequency of the tubes by adjusting the sleeves surrounding the tube. The boundary conditions for such a suspension system are not adequately clear and might result in difficulties while modeling the system. The tubes were $1.19 \mathrm{~m}$ long with a diameter of $0.019 \mathrm{~m}$ and were arranged in a normal square array having a $P / D$ ratio of 1.44 . The frequency of the tubes was adjusted to $75 \pm 1 \mathrm{~Hz}$. The maximum mass flow rates through the $1.19 \mathrm{~m} \times 0.273 \mathrm{~m}$ channel were $15 \mathrm{~kg} / \mathrm{s}, 670$ $\mathrm{kg} / \mathrm{s}$ and $28 \mathrm{~kg} / \mathrm{s}$ for air, water and steam respectively. The steam was generated in an experimental nuclear reactor at a maximum temperature and pressure of $210^{\circ} \mathrm{C}$ and 25 bar respectively. The range of void fractions for the steam-water tests was $51.7-100 \%$. Spatially averaged void fraction measurements were made using 10 gamma-ray densitometers positioned upstream and downstream of the tube array. The vibration was measured using biaxial accelerometers and strain gages located inside specific test tubes. Instability was clearly seen in both air-water and steam-water flow cases. Their experiments revealed similar damping values in both steam-water and air-water flow. The experimentally measured two-phase damping values showed a strong dependence on the void fraction with a maximum of about 0.02 within a void fraction range of $40-70 \%$, and lower values below and above this range of void fractions. Other researchers have reported this observation too. Based on their experiments, a value of 7.2 was recommended for $K$. The authors extended their work to triangular arrays in a subsequent work (Axisa et al., 1985) and found similar behavior for both square and triangular arrays. This is in contradiction to the results of Pettigrew et al who see a dependence of the instability on the geometry of the tube array. However, in this paper, they revised their $K$ value to between 4 and 5 . 
Nakamura, Fujita, et al. (1995) conducted steam-water experiments at extreme pressures and temperatures of $7 \mathrm{MPa}$ and $284^{\circ} \mathrm{C}$ respectively. Tests were conducted on short span tubes $(0.174 \mathrm{~m}$ in length) and long span tubes $(0.5 \mathrm{~m}$ in length) having a diameter of $0.019 \mathrm{~m}$. The tubes were arranged in a $5 \times 20$ square array having a pitch of $0.027 \mathrm{~m}$. A $3 \times 3$ array of tubes in the center was made flexible by suspending from the sides using stainless steel piano wires. For the short span tests, the natural frequency of the tubes were adjusted to $18 \mathrm{~Hz}$ in air, but dropped to $12.9-14.5 \mathrm{~Hz}$ in water. The corresponding damping ratios were $5-11 \%$. The natural frequency and damping were $11.3 \mathrm{~Hz}$ and $3 \%$ respectively for the long span tests. The maximum gap velocity, $V_{r}$, was $1.3 \mathrm{~m} / \mathrm{s}$ for water flow and varied from $3.5-6.3 \mathrm{~m} / \mathrm{s}$ for steam flow depending on the temperature and pressure conditions. In their experiments, the gas flow velocity was held constant while gradually increasing the liquid velocity till the point of instability. Instability was clearly seen in most of the steam-water experiments. The authors presented a new theory for correlating the instability data based on equating the energy of the fluid to that of the tube oscillation. The correlation seems to provide a satisfactory stability boundary that works for the authors' data and also those of some other experimenters. Recently, the same group (Nakamura et al., Hirota et al., Mureithi et al., 2002) has completed a series of tests providing some much needed data on damping and fluid-elastic instability in steam-water two-phase flow. Tests were carried out on an inline array and an array inclined at $30^{\circ}$ to the direction of the flow. The array was essentially rigid with the exception of a few test tubes located at the beginning, center and the end of the array. Electromagnets were used to excite the tubes to study damping characteristics. In their work, the authors used homogenous properties to calculate the flow parameters such as density, velocity and void fraction. Their tests indicated the damping ratio of the tubes in the drag direction to be almost double of that in the lift direction, which also supported their observation of fluid-elastic instability being achieved earlier in the lift direction. They also reported that the damping of the tubes located at the two extremities of the array was higher than the one in the center that was attributed to the entrance and exit effects. They also reported higher damping ratios for the inclined array as compared to the normal array though an explanation for this behavior was not provided. The fluid-elastic instability tests indicate two peaks in the tube amplitude for the tube located in the first row of the array. The first peak was attributed to periodic vortex shedding and the latter stronger peak was attributed to the fluid-elastic instability. Interestingly, the peak corresponding to the vortex shedding was absent for the tube located in the center of the array. Their results also indicated that the critical reduced velocity decreased with an increase in system pressure of the steam-water mixture. However, the critical reduced velocity did not change significantly with void fraction.

Joo and Dhir (1994, 1995) investigated fluid-elastic instability in air-water flow and proposed a mechanistic model for the instability based on the forces acting on the tube. Their work included measurement of the drag coefficients and the fluid forces acting on the tubes. The experiments were carried out in an acrylic test section having dimensions of $0.125 \mathrm{~m} \times 0.2 \mathrm{~m}$. Rigid acrylic tubes, $0.2 \mathrm{~m}$ in length and $0.022 \mathrm{~m}$ in diameter were arranged in a normal triangular array with one flexible test tube in the center of the array which was suspended from the walls of the test section using cantilever beams. This design limited the vibration of the tube in the direction normal to 
the cantilever beams. However, by changing the orientation of the cantilever beams with respect to the flow, vibration in both the lift and the drag directions were measured separately. The natural frequency and damping of the tubes were changed by changing the thickness of the cantilever beams and the mass of the tubes. The tubes vibrated with a natural frequency of $8.5,12.2$, or $17.4 \mathrm{~Hz}$ in water. Pitch-to-diameter ratios of 1.3 and 1.4 were tested. Strain gages bonded to the cantilever beams were used to measure the vibration of the tubes, and also the transient forces based on the mean value of the strain gage signal. Detailed experiments were conducted to study the void fraction distribution around a test cylinder using a gamma ray densitometer. Pressure distribution and hence drag coefficient around the cylinder was measured by installing pressure taps around the periphery of the tube. The two-phase instability point was reached by maintaining a constant water flow rate, lower than the single-phase instability value, and gradually increasing the air flow rate. Their experiments revealed that the critical reduced velocity was a function of both the void fraction and the liquid reduced velocity. For the same void fraction, the instability was realized earlier for a lower liquid reduced velocity. Damping ratios measured from the spectrum of the strain gage signal in flowing twophase flows were found to be higher than still fluid as observed by previous researchers. Their experiments suggest a value of $5.4-8.2$ for $K$. The void fraction measurements revealed a scarcity of voids near the forward stagnation point of the test cylinder.

Pettigrew, Taylor et al $(1994,1998)$ present a good review of the literature on fluid-elastic instability in two-phase flows. As in the single-phase flow cases, the authors compiled a database of experimental points and correlated them to determine a stability bound that can act as a design guideline for heat exchangers. This resulted in a value of 3 for the instability constant, $K$. The instability map for continuous two-phase flows appearing in their paper is reproduced in Figure 1.3. The graph shows significant scatter and does not point out the effect of the void fraction or the flow regime on the instability. The authors indicate the need for further understanding of the phenomenon of fluidelastic instability in two-phase flows, especially the effect of the flow regime on instability. A recent article by Weaver, Ziada et al (2000) regarding the state-of-art of fluid-elastic instability studies in two-phase flow indicates the need for studying the effect of void fraction and flow regime on the instability. 
FLUIDELASTIC INSTABILITY RESULT

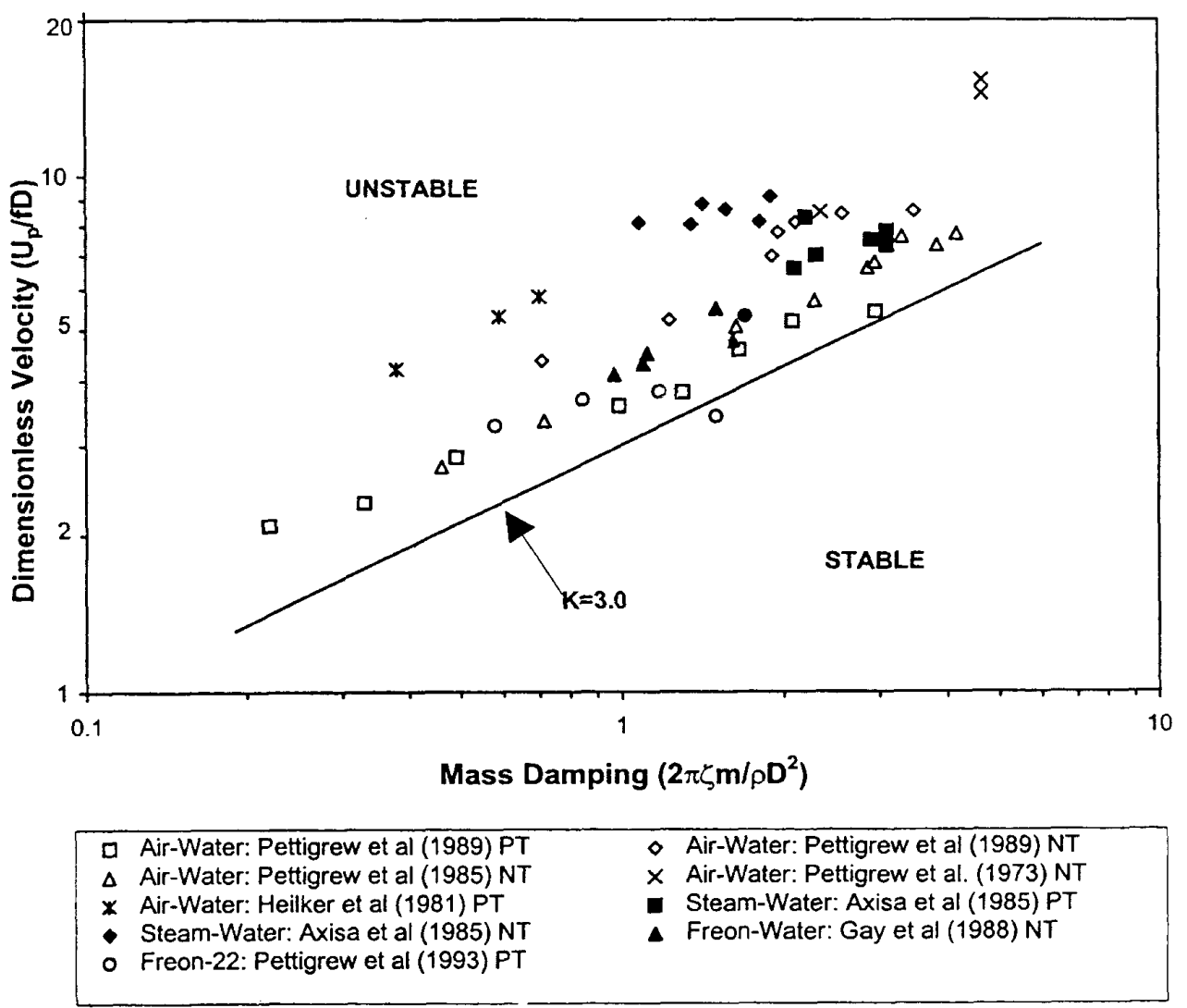

Figure 1.3: Fluid-elastic Instability in continuous two-phase flows (Reproduced from Pettigrew et al., 1998)

Since the publication of the above mentioned review papers, some work has been carried out to study the effect of void fraction and flow regime on instability. Taylor and Pettigrew (2001) compare various void fraction models and point out that the homogeneous model often used by researchers to calculate flow parameters almost always overpredicts the void fraction encountered in the experimental test section. This is due to the unaccounted slip between the two phases. Their study indicates that a model incorporating the slip such as the drift-flux model or the Schrage model better predicts void fraction measurements made using radiation attenuation equipment such as gamma densitometers. Their measurements reveal that the void fraction increases through the tube array due to the pressure drop through the bundle. At high void fractions above $80 \%$, there is a sudden decrease in the critical reduced velocity. This had previously been seen by Pettigrew as well as other researchers and is attributed by the authors to a change in the flow regime from bubbly flow to intermittent flow.

Feenstra, Weaver and Judd (2002, 2002, ref) recently carried out experiments using Freon 11 with an objective to develop a methodology for predicting critical velocities for fluid-elastic instability based on the physics of the phenomenon. The contribution of this paper is a new method for calculating the average density of the mixture and an equivalent flow velocity using a newly developed void fraction model that allows for slip. The equivalent velocity is based on the assumption that a reasonable 
measure of the fluid-elastic excitation is provided by the total kinetic energy of the two phases. They express the equivalent velocity as

$$
V_{e q}=\sqrt{\frac{\alpha \rho_{g} U_{g}^{2}+(1-\alpha) \rho_{l} U_{l}^{2}}{\rho}}
$$

where $\alpha$ is the void fraction, $\rho_{g}$ and $\rho_{l}$ are density of the gas and liquid phases respectively, $U_{g}$ and $U_{l}$ are the superficial velocities of the gas and liquid phases, and $\rho$ is the equivalent density. An instability map based on these flow parameters helps to collapse the mass damping parameters for the different flow regimes.

\begin{tabular}{|l|l|l|l|l|l|}
\hline & $\begin{array}{l}\text { Axisa } \text { et al. } \\
\mathbf{( 1 9 8 5 )}\end{array}$ & $\begin{array}{l}\text { Pettigrew } \text { et } \\
\text { al. } \mathbf{( 1 9 8 9 )}\end{array}$ & $\begin{array}{l}\text { Joo and } \\
\text { Dhir } \\
\mathbf{( 1 9 9 5 )}\end{array}$ & $\begin{array}{l}\text { Feenstra } \text { et } \\
\text { al. (1995) }\end{array}$ & $\begin{array}{l}\text { Hirota } \text { et } \\
\text { al. (2002) }\end{array}$ \\
\hline Fluid & $\begin{array}{l}\text { Steam- } \\
\text { Water }\end{array}$ & Air-Water & Air-Water & R-11 & $\begin{array}{l}\text { Steam- } \\
\text { Water }\end{array}$ \\
\hline $\begin{array}{l}\text { Array } \\
\text { Geometry }\end{array}$ & $\begin{array}{l}\text { Triangular, } \\
\text { Normal } \\
\text { square }\end{array}$ & $\begin{array}{l}\text { Normal } \\
\text { triangular, } \\
\text { square }\end{array}$ & $\begin{array}{l}\text { Normal } \\
\text { triangular }\end{array}$ & $\begin{array}{l}\text { Parralel } \\
\text { triangular }\end{array}$ & $\begin{array}{l}\text { Normal } \\
\text { square }\end{array}$ \\
\hline P/D range & 1.44 & $1.32,1.47$ & 1.4 & 1.44 & 1.46 \\
\hline $\begin{array}{l}\text { Void } \\
\text { fraction (\%) } \\
\text { range }\end{array}$ & $85-98$ & $5-99$ & $0-50$ & $0-80$ & $70-96$ \\
\hline $\begin{array}{l}\text { Method of } \\
\text { suspension }\end{array}$ & $\begin{array}{l}\text { Cantilevered } \\
\text { at end }\end{array}$ & $\begin{array}{l}\text { Cantilevered } \\
\text { at end }\end{array}$ & $\begin{array}{l}\text { Cantilever- } \\
\text { beam sus. }\end{array}$ & $\begin{array}{l}\text { Cantilevered } \\
\text { at end }\end{array}$ & $\begin{array}{l}\text { Piano } \\
\text { wire }\end{array}$ \\
\hline $\begin{array}{l}\text { Rigid/Flex. } \\
\text { array }\end{array}$ & $\begin{array}{l}\text { Fully } \\
\text { Flexible } \\
\text { array }\end{array}$ & $\begin{array}{l}\text { Fully } \\
\text { Flexible } \\
\text { array }\end{array}$ & $\begin{array}{l}\text { Flexible } \\
\text { tube/Rigid } \\
\text { array }\end{array}$ & $\begin{array}{l}\text { Fully } \\
\text { flexible } \\
\text { array }\end{array}$ & $\begin{array}{l}\text { Flexible } \\
\text { tube/Rigid } \\
\text { array }\end{array}$ \\
\hline L/D & 62.5 & 46 & 9 & 48 & 7.25 \\
\hline
\end{tabular}

Qualitative differences in the instability results with different fluid systems are noticed when comparing experimental data available in literature. This is shown for a limited set of data in Fig. 1 where the onset of instability is plotted using the Connors' criterion. The experimental conditions for each data set are shown in Table 1. The experiments differ in the fluid systems, and also in the structural details of the system such as the manner of tube suspension and the ratio of the length to diameter of the tubes. The second and third factors affect the vibration characteristics of the tubes and are not directly accounted for in the Connors' criterion. The trends indicate that in air-water systems, a fully flexible array becomes unstable earlier as compared to a single flexible tube in a rigid array. 


\subsection{Modeling}

This component of the project deals with the development of an accurate theoretical model that can be used to predict fluid-elastic instability. To this end, the aim of the model would be to predict the amplitude of tube vibration of a set of tubes, and to determine the onset of instability based on a stability analysis.

Theoretical modeling has been addressed in the past in a few studies. However, most of them have been found to be lacking in some form or another. In the case of a fluid-structure interaction problem, the elements of the model consist of a fluid component and a structural component, both of which will be treated equally. Such an approach is not usually found in the literature and the success of the model in accurately predicting the instability lies in the accurate treatment of both these components.

Most of the theoretical studies in fluid-elastic instability have been carried out for single phase flow. Modeling two-phase flow across rod bundles is a complex subject. The vapor, or other gas phase, rises due to gravity and experiences drag normal to gravity by the liquid phase when the tube orientation is vertical. In particular, at the top of a U-tube steam generator, the tubes themselves are at an angle relative to gravity making the problem even more complex. As a result, the usual drift flux approaches are not adequate to predict gross characteristics of the flow. To further compound the problem, the equations for such flows are not agreed on by the two-phase flow research community.

The approach that will be taken for modeling two-phase flow is modest. Marn (1991) and Marn and Catton (1991) developed some numerical methods for modeling fluid-elastic instability. Their two-phase model was modest and developed for air-water flow. The governing equations for the fluid were based on the two-fluid representation using the TRAC equations. The structural equations were derived by treating the tubes as a spring-mass-damper systems. The equations of fluid motion and the tube motion are coupled at the fluid-structure interface by pressure, friction, and tube motion boundary conditions. The void fraction of the mixture appears as a parameter in the fluid equations. Two dimensional normal modes were used for analyzing an array of tubes all of which were subjected to perturbations of different kinds. They demonstrated that the feedback between the structure and the fluid is the main contributor to a growing solution of coupled solid-fluid equations of the tube's motion. The results of their analysis suggested that instability occurred earliest when all the tubes in the array were subjected to different perturbations indicating that the tubes do not vibrate in-phase and in the same manner in the post critical stages. This has been seen in some of the experiments especially in the single phase flow cases. The results of Marn and Catton are shown below in Figure. 11. 
Reduced Velocity vs. Mass Damping Parameter

Finite Array, Str. Re $=400,000$

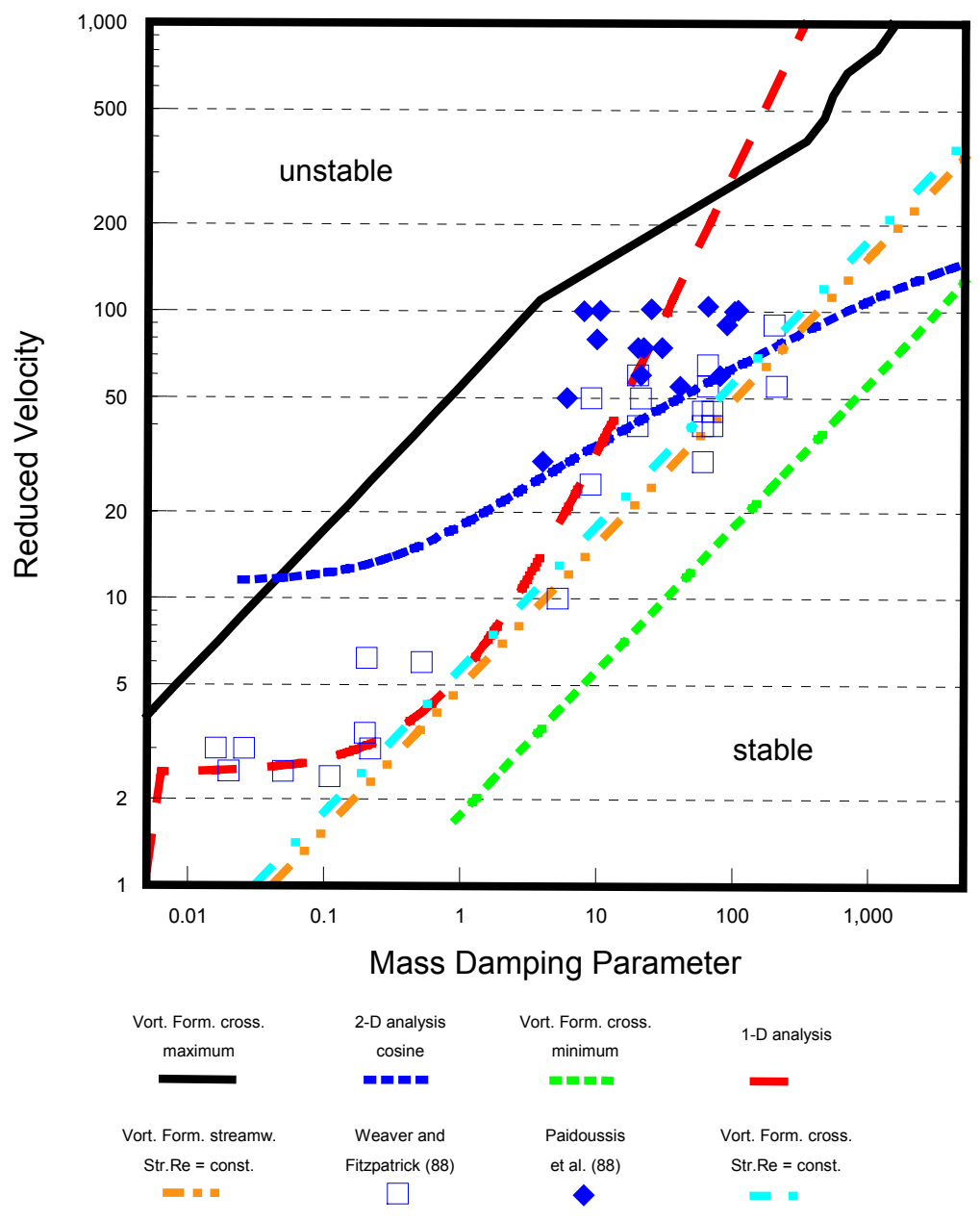

Figure 11. Theoretical stability analysis from Marn and Catton
As real time three-dimensional simulation of the phenomenon is far too complicated at this stage, the most promising approach would be to break the phenomena into its accompanying mechanisms and analyze only the most important. Previous work and the authors' experience points at the fluid-elastic forces as the most important cause of the instability. To this end, the focus will be to find the correct set of governing equations and accompanying boundary conditions which would lead to a robust tool. The airwater flow problem will be revisited and Marn and Catton's existing work will be extended to include arrays having different pitch-to-diameter ratios and array geometries.

Different void fraction models which are now available in the literature due to a paper by Taylor and Pettigrew (2001) will be evaluated. In addition, the code will be generalized to include the effects of gravity, implying a vertically upwards flow as occurs in the experiments in the Boiling Heat Transfer Laboratory. The gravity would affect slip between the liquid and gaseous phases and as such possibly change the assumptions used in the development of the model. Furthermore, it has been shown that in the bubbly and slug flow regime that occurs in the steam generators at the onset of instability, gravity does play a deciding role.

In the first model, the void fraction was assumed to be invariant in a computational streamtube surrounding the tube. However, Joo and Dhir (1995) have shown that a void fraction profile exists close to the tube and varies circumferentially and 
radially as shown in previous sections. Clearly, there is room for improvement in this regard in the previous model.

One of the main goals of the modeling will be to include effects due to a fluid systems consisting of a fluid and its own vapor. The examples of such systems are steamwater and Freon-freon vapor. The steam-water fluid system is the truest representation of flow in a steam generator. However, modeling such a system is complicated due to the coupling of the energy and momentum equations. Furthermore, if boiling occurs on the tube surface, there will be mass transfer of vapor at the interface. Constitutive relations for heat and mass transfer must be added to the governing equations and the vapor distribution in the flow channels could be very different. The simplest assumption would be to break the flow field into three layers as shown in Figure. 12. In such a model, the vapor is present as a continuous layer adjacent to the tubes with liquid or two-phase mixture present in the central layer. As a second attempt, the void fraction profiles obtained from the experiments with steam-water flow and boiling obtained in the earlier phase of the project will be used to determine a suitable model for the fluid flow. In reality, the fluid changes phase as a result of local temperature and pressure changes in the flow field. In steam-water flow, a vapor bubble or slug can expand or shrink as a result of this phase change and hence alter the coupling between the structures. Hence, the model should be able to incorporate this effect. Experiments with steam-water would give some insights into the significance of the above mechanism thus suggesting improvements to the model.

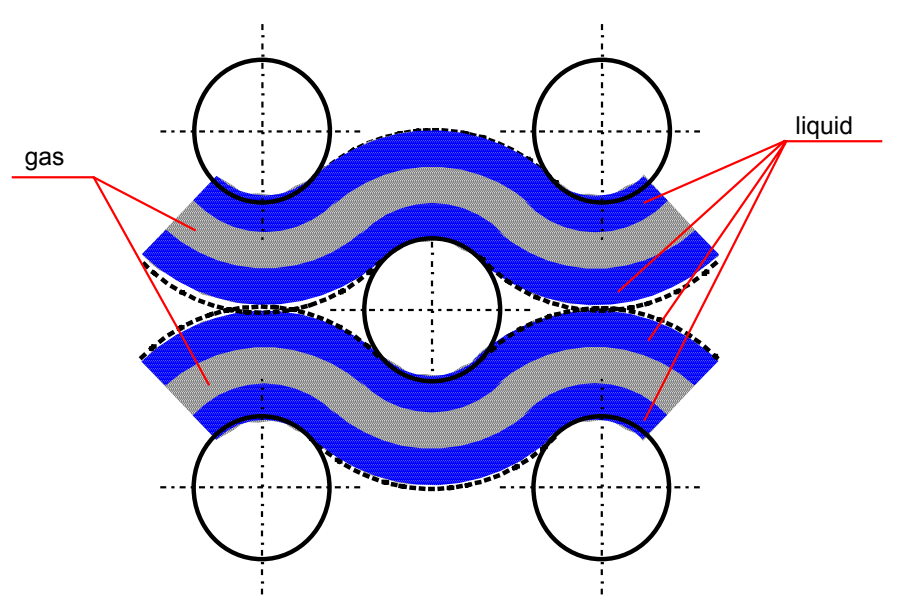

Figure 11. Three layer concept.

\subsection{Objective of Present Study}

A review of the literature on fluid-elastic instability shows no unanimous agreement on the design criteria for heat exchangers. This can be easily seen from the wide range of the values of the instability constant reported by different researchers. A conservative value for the instability constant will result in economic penalties in terms of design, whereas under-designed heat exchangers run the risk of failure during 
operation. There is no uniform system for reporting measured experimental parameters making the task for designers and modelers even more difficult.

In the pursuit of a complete description of the fluid-elastic instability, the singlephase flow experiment seeks to observe and quantify two essential aspects of the phenomenon: the instantaneous velocity field surrounding the tube bundle and, simultaneously, the real-time displacements of each tube in the bundle. These measurements are necessary in determining the relative influence of each structural and fluid parameter towards the fluid-elastic instability, by way of decoupling the fluid forces and structural response in the governing dynamical equations for each tube. Simultaneous measurements of the fluid velocity field and structural response for multiple tubes have not been achieved in any reported experiment so far. Thus, the single-phase study proposes to obtain this new information not only for the development of more useful design criteria for heat exchangers and modeling, but also for the advancement of the understanding of fluid-elastic instability phenomena in general. Data obtained from the experiment will be applicable for comparison with several other studies, and provide complete results for FEI in a multiple tube system, so far unavailable.

Finally, the investigation of the phenomenon for two-phase flows is still not understood well enough and has scope for further development. Specifically, the areas that still need to be addressed are instabilities in steam-water flow, and the effect of the void fraction and flow regime on the instability. The objective of the present work is to revisit the problem of fluid-elastic instability for two-phase steam-water and air-water flow and conduct systematic experiments to determine experimental parameters at the onset of instability. Parameters to be measured include the flow velocity, frequency of vibration of the tubes, damping ratio of the tubes, and the void fraction of the mixture. Experiments will be carried out on an array of flexible tubes suspended using piano wires. Most of the experiments on fluid-elastic instability will be conducted on a normal square array and a rotated square array of pitch-to-diameter ratio of 1.4. Baseline measurements of the critical flow velocity required to achieve instability in water will first be made followed by two-phase tests with air-water and steam-water flow. In the two-phase flow tests, the water flow rate will be held constant at $25 \%$ or $75 \%$ of the single phase critical flow rate and the gas or vapor flow rate will be gradually increased to the point of instability. The vibrations will be measured using strain gages bonded to the piano wires. The effect of pitch-to-diameter ratio on the instability will be investigated by changing the pitch of the tube array to obtain $P / D$ ratios of $1.2,1.4$ and 1.55 for the normal square array. Experiments will be carried out to study the effect of nucleate boiling on the tube surface in steam water flow. This experiment will be carried out using cylindrical cartridge heaters as heated tubes. Limited experiments will be carried out to study the circumferential and radial void fraction distribution around a rigidly held tube in a normal square tube array in steam-water flow. Since the drag coefficient is an important parameter for modeling the instability mechanism, some experiments will be carried out to determine the pressure distribution around a rigid tube instrumented with pressure taps around its periphery. This experiment will be carried out with steam-water flow. 
The goal of this research is to provide accurate measurements that can help modelers to validate their models using the measured experimental parameters and thereby develop better design criteria for avoiding fluid-elastic instability in heat exchangers.

The outline of this report is as follows: After the introduction to fluid-elastic instability, the experimental apparatus constructed to conduct the experiments is described in Chapter 2 along with the measurement procedures. Chapter 3 presents results obtained on the tube array and the flow loop, as well as techniques used in data processing. The project performance is described and evaluated in Chapter 4 followed by a discussion of publications and presentations relevant to the project in Chapter 5, while the conclusions and recommendations for future work are presented in Chapter 6. 


\section{Experimental Apparatus}

The single phase experiments are focused on gathering displacement data for different patterns of multiple tubes in water cross-flow. By varying the structural and geometric parameters describing the tube bundle, the influence of these parameters on the onset of fluid-elastic instability may be determined. Two different test sections, each containing the tube array in a different configuration, have been designed and constructed. Each configuration of the tube array has been observed under controlled flow conditions. In order to obtain the needed flow conditions, a specifically designed flow loop has been set up. Measurements of tube displacements have been made using an optically based data acquisition system. The image data obtained from the experiments has been reduced using computer programs to process and analyze the images. The resulting information can then be applied to the modeling of the structural dynamics of the instability.

The two-phase flow loop is different from the one used for the single phase flow experiments. For the two-phase experiments, the direction of the flow is vertically upwards as compared to the horizontal flow direction for the single phase flow experiments. The purpose of this was to avoid horizontal two-phase flow during the experiments. In addition, the construction of the test section features stainless steel to accommodate the high temperatures of steam-water flow.

\subsection{Single Phase Experiment}

\subsubsection{Flow Loop Description}

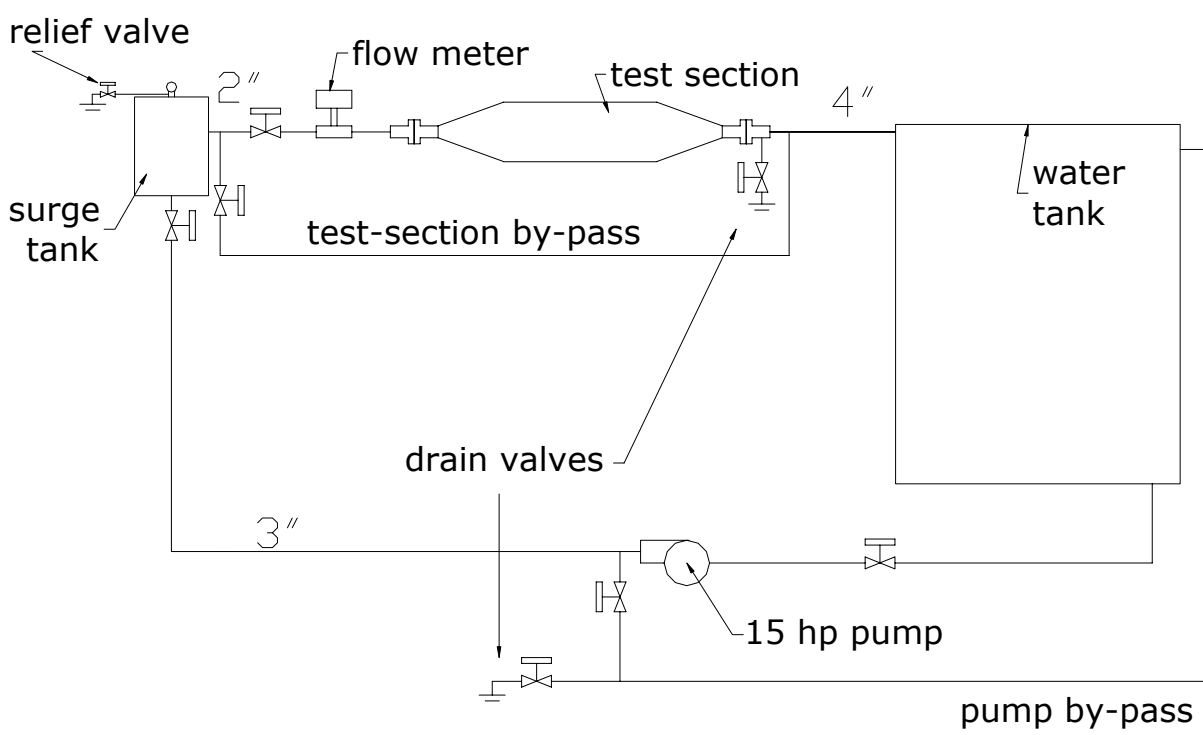

Figure 2.1. Schematic of the flow-loop. 
The flow loop consists of four main parts: a reservoir, a pump, a surge tank and the test section, which is described in detail later on. A schematic of the flow loop is shown in Figure 2.1. The water stored in the main reservoir is drawn through a 4-inch pipe located on the tank bottom. A $15 \mathrm{hp}$ centrifugal pump provides the pressure to drive the fluid flow throughout the loop. A valve is located between the pump and reservoir, providing a direct means to regulate the supply of water. The pump outlet and delivery line are 3" PVC pipe. Another valve is located after the pump and a second valve permits drainage via the discharge line, before the water enters into the surge tank. Furthermore, a by-pass line is used to control the flow rate directly downstream of the pump, allowing for part of the flow to be diverted back to the reservoir. The surge tank is designed to create a homogenous flow profile for the test section and to damp the oscillations in the flow caused by the pump. It is fitted with a relief valve to allow for air to exit the system. Downstream from the surge tank, the fluid enters the test section after passing a control valve; an in-line differential-pressure flow meter (McCrometer V-Cone, model 3051) was installed in order to assure an accurate measurement of the volumetric flow rate, Figure 2.2. The flow meter output is in a range between 4 (corresponding to 0 gallons per minute) and $20 \mathrm{~mA}$; the signal is then converted to a percentage of the maximum flow rate using the following formula:

$$
\text { Flowrate }[\%]=\left(\frac{X m A-4 m A}{16 m A}\right)^{2}
$$

The maximum acceptable flow rate is 300 GPM (gallons per minute). The accuracy of the instrument is $0.075 \%$ of the calibrated span. The test section can be drained separately from the system and is equipped with a relief valve to release the surplus air and absorb pressure spikes. A second by-pass circuit is provided in order to control the flow rate in the test section. Downstream of the test section, the water flows through the return line, which has an increased diameter of 4" to minimize the back pressure at the test section, and is discharged into the main water tank.

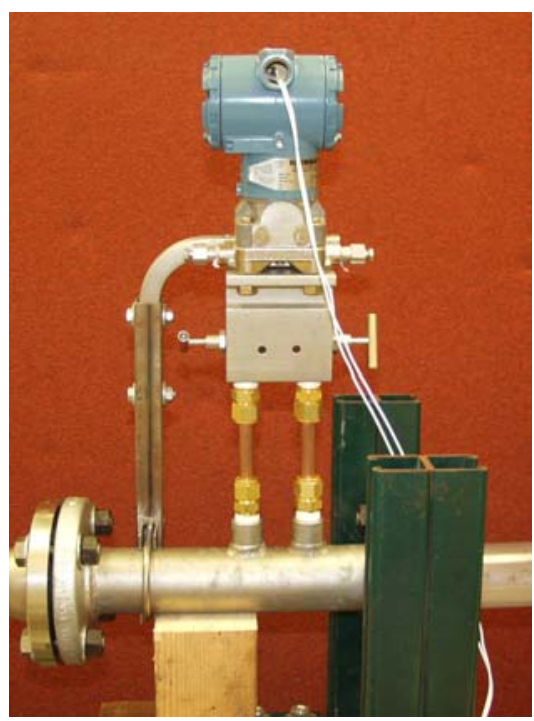

Figure 2.2. Differential-pressure flow meter 
The dimensions of the main water tank are 46 inches in diameter, 72 inches in height and 1/8 inch thick. The volume is 517.75 gal. The pump is rated at $15 \mathrm{hp}$ and can potentially discharge 400 gallons per minute at 3485 RPM. The pipes of the flow loop are 2 and 3 inch PVC, rated schedule 40. They are welded at both ends to PVC flanges for easy installation and disassembly. The pump suction line has an inner diameter of 4 inches, while the discharge line has an inner diameter of 3 inches. The surge tank is made of stainless steel and its dimensions are 30 inches high, 22.5 inches in diameter and $1 / 8$ inch thick, with a capacity of 51.6 gallons. The total length of the flow loop, from the pump to the exit in the main water tank is approximately 28 feet. The maximum flow rate achieved was 270 gallons per minute, with a maximum velocity between the tubes of $1.78 \mathrm{~m} / \mathrm{s}$.

\subsubsection{Test Section}

The test section consists of two stainless steel diffusers separated by an acrylic main box where the tubes, the objects of the measurements, are placed. The inlet diffuser (Fig. 2.3) is designed so that the flow can develop from the 2-inch pipe and enter the rectangular test section. The angle of the diffusers is 7 degrees, a standard design to prevent flow separation. At the end of the angled part of the diffusers there is a straight section which houses a standard aluminum honeycomb structure, 3 inches in cell depth, to straighten the incoming flow. The outlet diffuser is fitted with a 3-inch flange at the end to connect to the return line. To connect the diffusers to the test section, a bolt flange is welded at the end of each diffuser.

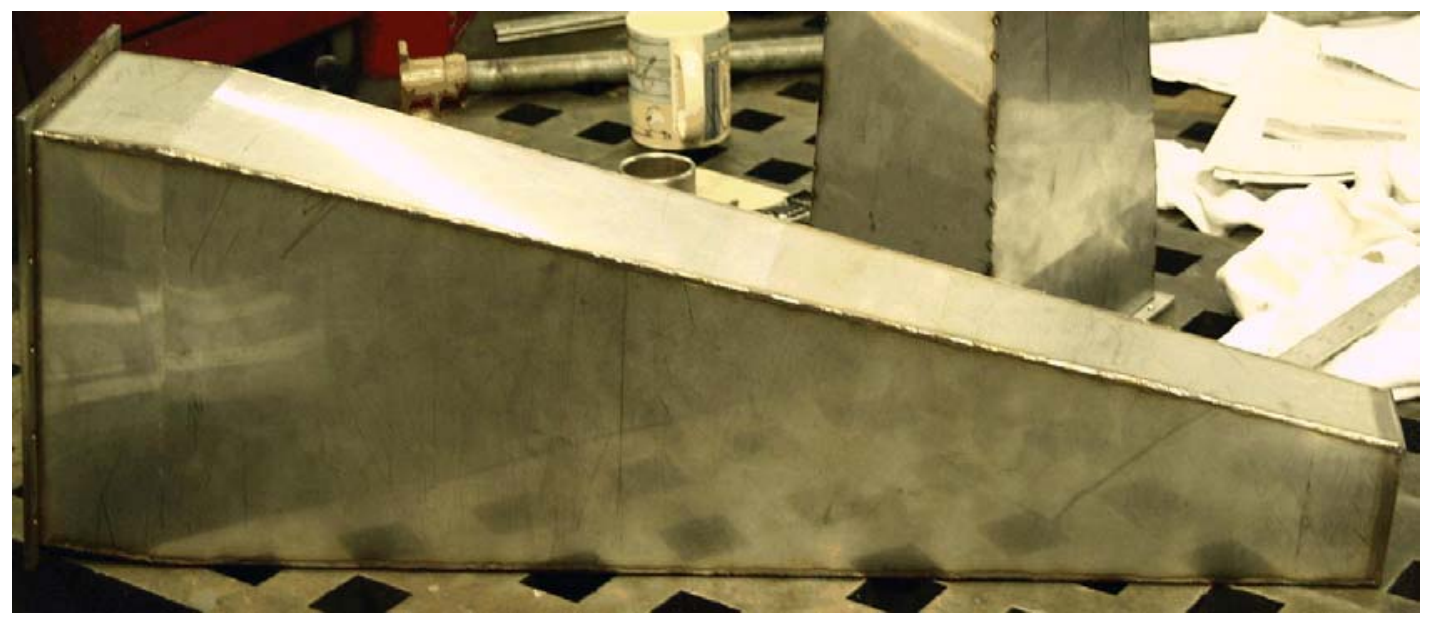

Figure 2.3. Stainless steel diffuser

The external dimensions of the acrylic test section are 16 inches in length, 6.6 inches high and 10.25 inches wide (Fig. 4.4). The top and bottom plates are 0.5 inches thick, while the side panels are 1 inch thick.

The test section includes two main parts. In the first part, a $3 \times 5$ square array of plain fixed acrylic tubes and 10 acrylic half-tubes are fitted in the walls of the box. The function of these fixed tubes is to develop the flow upstream of the vibrating tubes such 
that the vibrating tubes are subject to an oscillatory flow and not a uniform flow, which is hoped to more closely model the situation in actual heat exchangers. In the second part, a square array of $3 \times 5$ stainless steel tubes is suspended by stainless steel piano wire. These tubes are free both to vibrate linearly in the plane perpendicular to their axes, and to rotate about their axes. The vibrating tubes are the components for the actual heat exchanger model, and are thus the focus of the experimental observations. The stainless steel tubes have a 1-inch diameter and are 9.75 inches long. To allow space for installation of the tubes, a clearance of $1 / 8$ " is provided between each tube end and the box.

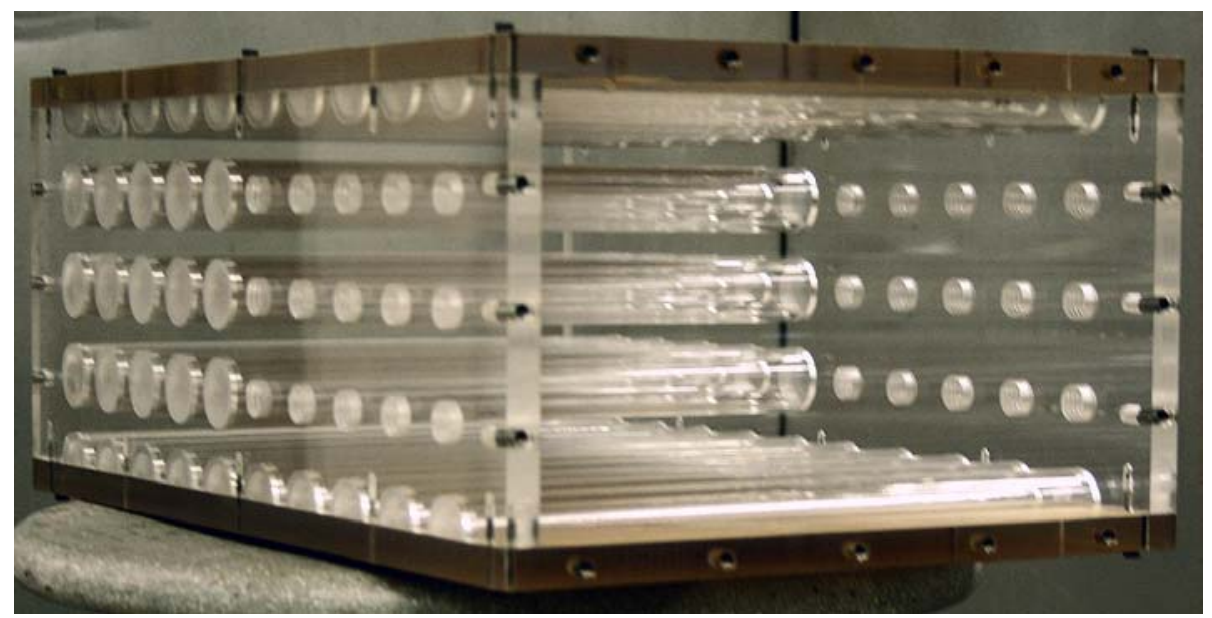

Figure 2.4 Acrylic test section

The inside bore of each vibrating tube is threaded and two $1 / 4$ inch thick disks are inserted by equal distances at each end. The disks provide fixed-point anchors for the piano wires, which are inserted through small clearance holes and fixed on the interior of the disks with an epoxy resin.

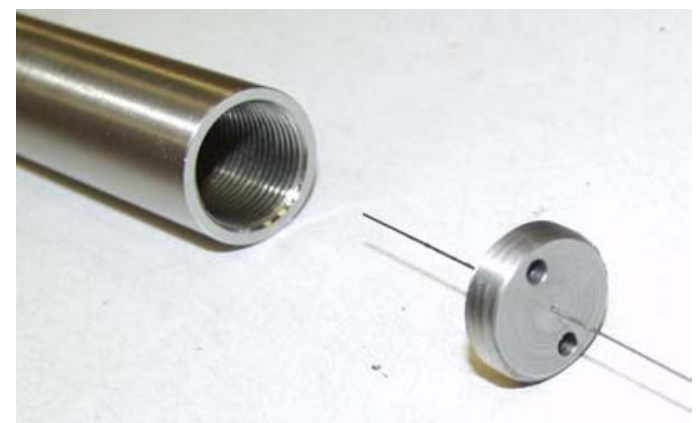

Figure 2.5 The piano wire connects to tube via the threaded disc insert

To one side of the test section, the side from which the tube array is viewed and observed, the piano wire is drawn through a vented bolt that is inserted via threads in the acrylic wall. An aluminum stopper placed on top of the screw is designed to hold the wire with a setscrew device and anchor the wire from this side (Figure 2.6). 


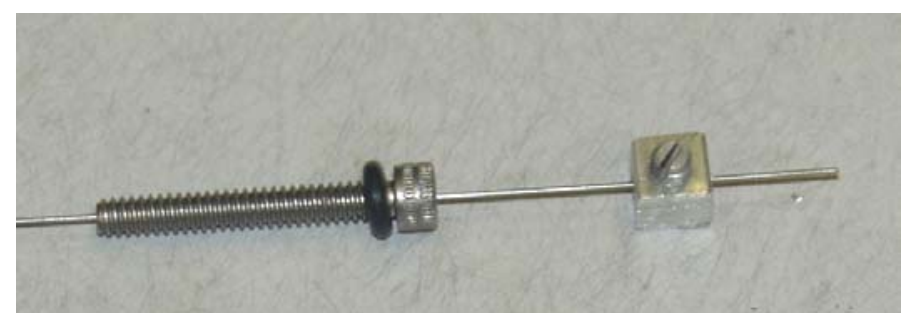

Figure 2.6. The piano wire support apparatus on the viewing side includes the vented bolt with an $O$ ring seal and a stopper with a set-screw device to hold the wire in place.

On the other side, the wire passes through another custom-made insert and is held by a cap placed on the top of a precision compression spring. The spring can be relaxed or compressed in order to change the tension of the wire. In this way it is possible to control the natural frequency of the suspended tube. Each of the tubes of the vibrating array is suspended in the test section in this manner, simulating the condition for vibration of the center portion of the heat exchanger tubes.

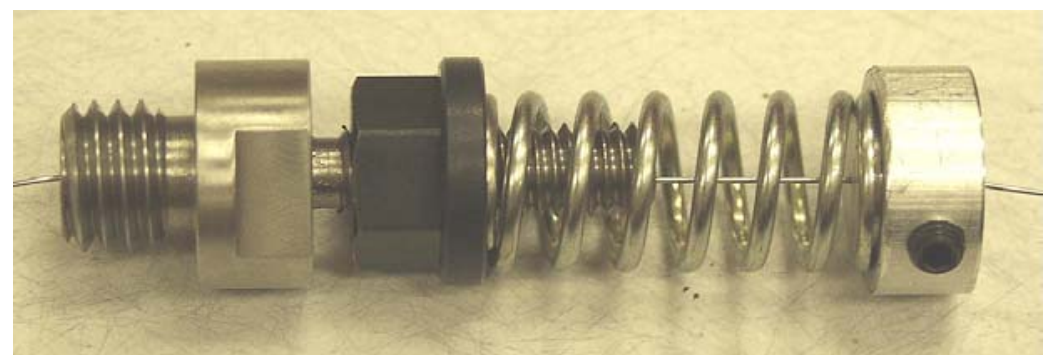

Figure 2.7. The remaining wire end is pulled through another threaded insert and attached to the tensioning mechanism

An overall picture of the tube apparatus is shown below (Figure 2.9). A schematic of the insert mechanisms used to support the tubes is shown in Figure 2.8.

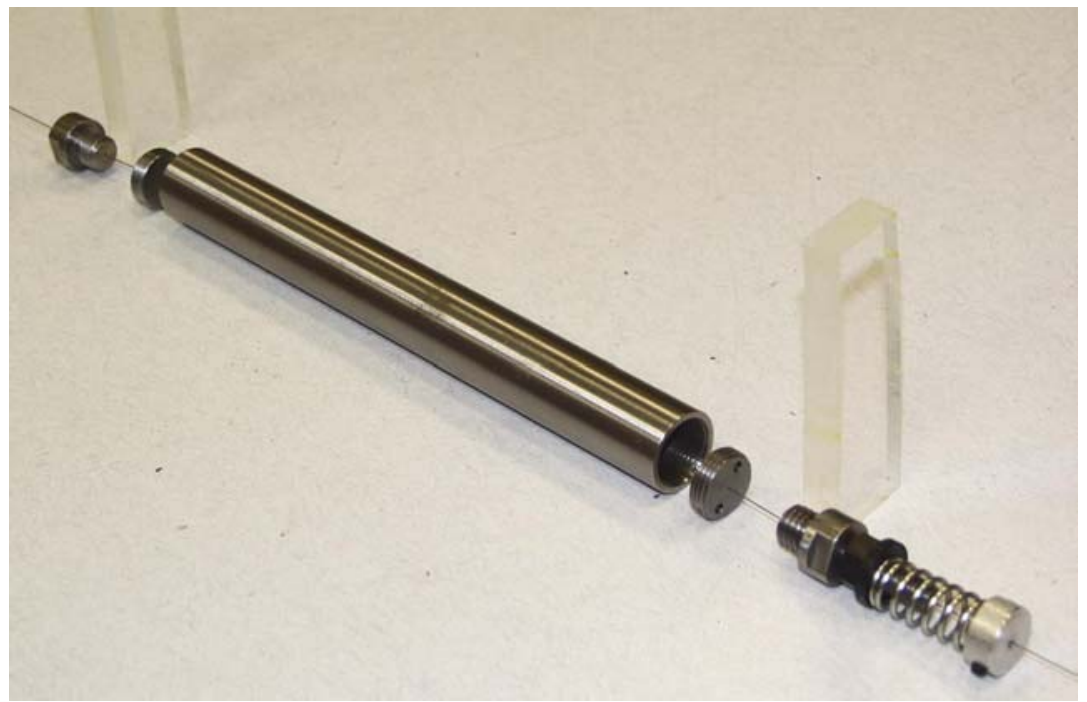

Figure 2.9 Overall vibrating tube configuration. 


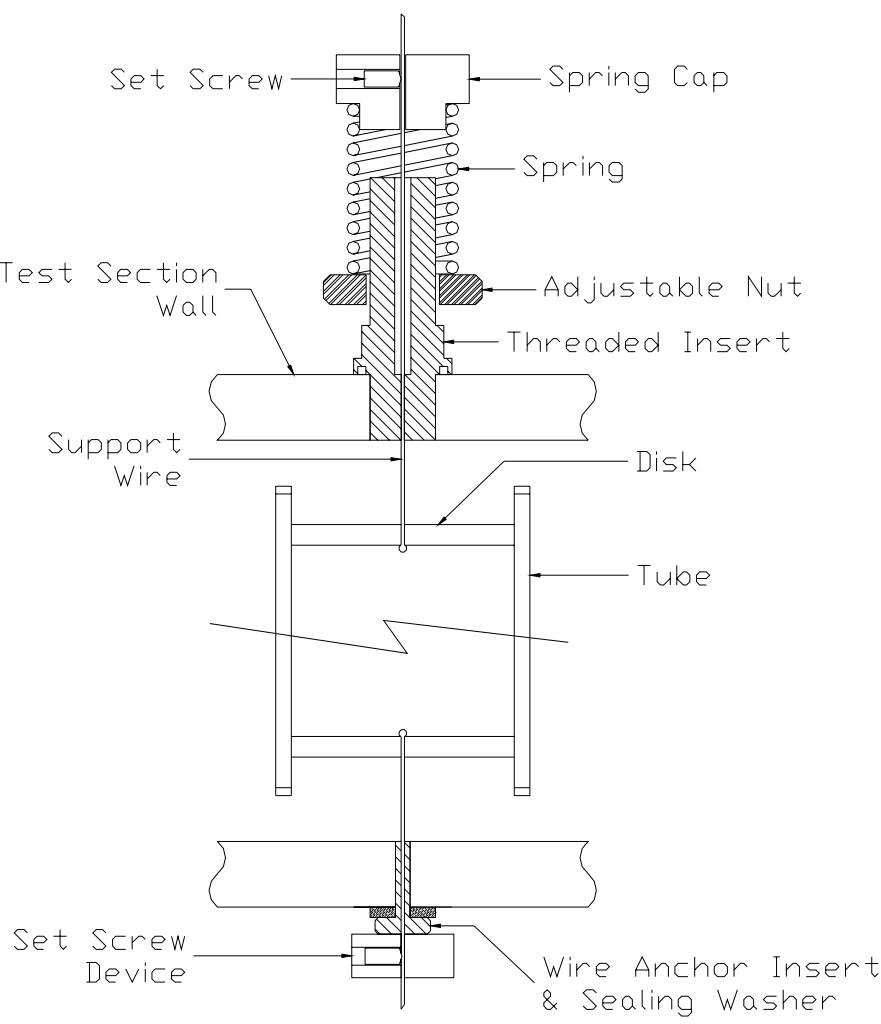

Figure 2.8. Diagram of the support and tension control mechanism for the flexible tubes

To prevent failure of the test section panels, an external structure was designed and fabricated to reinforce the test section against bending. The principle of this design was to develop a rigid structure, which could be easily attached to the existing test section and provide a direct support to the test section panels, maintaining the cross-sectional dimensions along the entire test section against the internal pressure forces. The support structure was designed so as not obstruct the view of the tube ends.

The support structure consists of five custom-made "clamping devices". Three of the clamps are designed for securing of the top and bottom test section panels, while the remaining two are placed laterally against the vertical side panels. The clamps are simply two opposing steel struts with holes at each end, through which two threaded steel rods are inserted. Nuts and washers are used to fix the length of rod between the two clamps on either side. This allows for the struts to be clamped together when placed on opposing faces of the test section. In order to distribute the force of the clamps and to allow for each of the clamp faces to contact the panel surface, thin metal plates (saddle plates) were cut to fit between the clamp faces and the test section panels. A thin rubber sheet was placed under each of the metal plates to allow for some small flexibility between the test section and the clamps, and to provide a self-adjusting surface on the panel to further distribute the force of the clamps evenly. The saddle plates were placed on all the test section panel faces. The top and bottom plates cover almost the entire panel surface, while four separate side plates were made and placed apart to provide an exposed view of the vibrating tube array (Figure 2.10). 


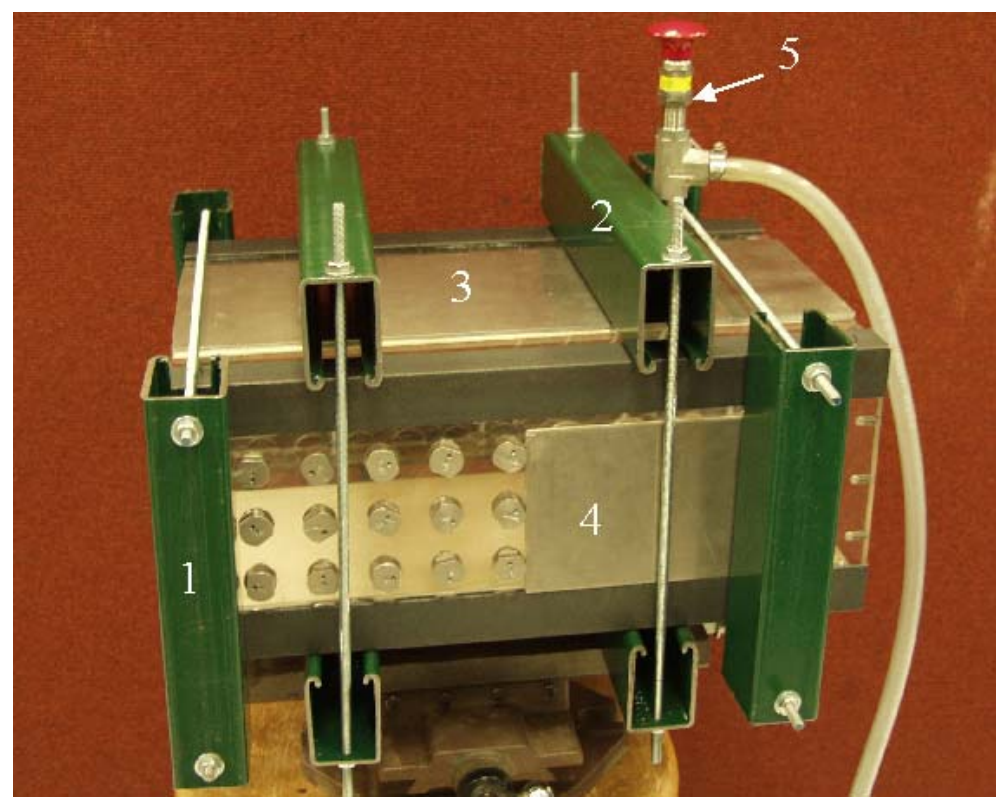

Figure 2.10. The reinforced test section. (1) Side clamp device, (2) top clamp device, (3) top saddle plate, (4) side saddle plate, (5) pressure relief valve

This reinforced configuration of the test section was installed and tested. While the test section showed no signs of deformation or failure at low flow rates, the diffuser bodies exhibited significant bending. It was concluded that the diffusers could not sustain pressures at the desired flow rates and would also need to be strengthened. The steel diffusers were similarly reinforced against bending by welding supporting struts at four locations along the diffuser. At each location, steel bars were welded around the entire perimeter of the diffuser body (on each of the four sides) to form a "frame" structure, with the same intention as per the test section reinforcement design. Furthermore, a port and boss for threaded fittings was added to the top face of each diffuser and controllable relief valves were installed as a secondary safety measure against pressure spikes. Pressure gauges have also been installed in-line with the relief valves. These gauges will give an indication of the pressure at both the inlet and outlet diffusers, allowing a rough estimate of the pressure drop through the test section.

The test section for the normal triangular array configuration is analogous to the design for the normal square array in almost every respect. The only parts that differ are the side panels. The array configuration for the normal triangular array consists of a flowdeveloping array of 17 fixed acrylic tubes ordered in 5 rows with ten half tubes, while the vibrating array includes 14 tubes arranged in 4 rows. The two configurations used in this experiment are shown in Figures 2.11a and 2.11b, below. 


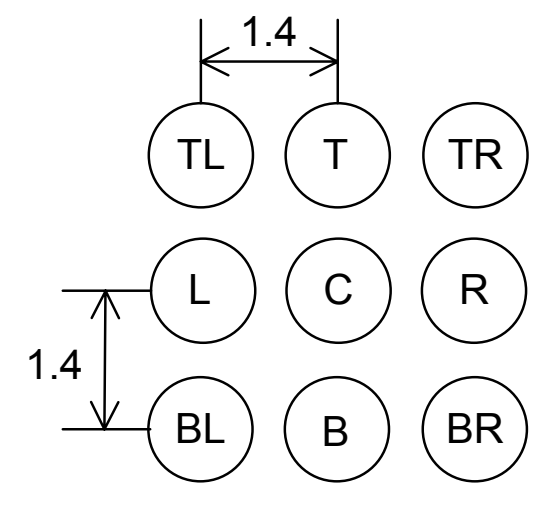

Figure 2.11 a) Normal square array configuration.

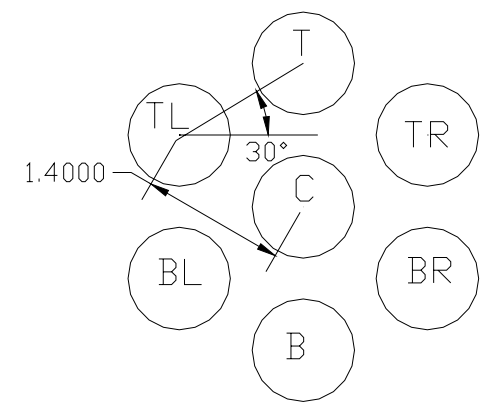

Figure 2.11 b) Normal triangular array configuration.

\subsubsection{Data Acquisition System}

The objective of the experiment is to study the vibrations of the tube array under flow conditions leading to the onset of fluid-elastic instability. The main effort has been directed towards the development and implementation of a system to obtain the timedependent displacement of the tube array subjected to various cross-flow velocities.

Using two high-speed digital cameras, sequential images of the tube array are captured for a period of vibration during which the tubes are excited by the water crossflow at a specific velocity. Sets of these "raw" (i.e. unprocessed) images, captured at a high time frequency, are basically movies of the tube motion at different flow speeds: one movie, equal to one thousand images (or about 8 seconds), is recorded for a specified mean flow speed. After some processing and analysis, the resulting data allows for the detailed inspection of the tube array vibration, in addition to the average vibration magnitude for a specified steady flow condition (i.e. for each flow speed). The cameras are CCD type sensors with a spatial resolution of 640 by 480 pixels and capture images at a rate of 120 frames per second. A major advantage of this data acquisition process is the fact that it is entirely non-intrusive; no sensors need to be placed inside or on the tube surfaces, and the problem of feeding wires through the test section is avoided. A reflective coating is applied to the ends of each free tube to enhance this imaging. It is also possible to suppress the brightness of the background when necessary. This optical adjustment to the captured images facilitates certain key aspects of the image processing stage later on.

The camera is situated facing one of the test section sides, so that the viewing plane of the camera is normal to the axis of the tubes (Figure 2.12). The frequency of the time series of images is determined by the highest expected frequency of tube vibration to be observed. By marking each of the tube ends at known angular locations, the axial 
rotations of the tubes may also be acquired. The rigid body motions of the tubes are thus calculated by comparing subsequent images.

The digital image capture consists of the transfer of the image from the optical system to a digital format that is saved directly to a hard drive. The camera takes an image of the tube array onto a charge- coupled device (CCD), which translates the optical signal into an 8-bit pixel array.

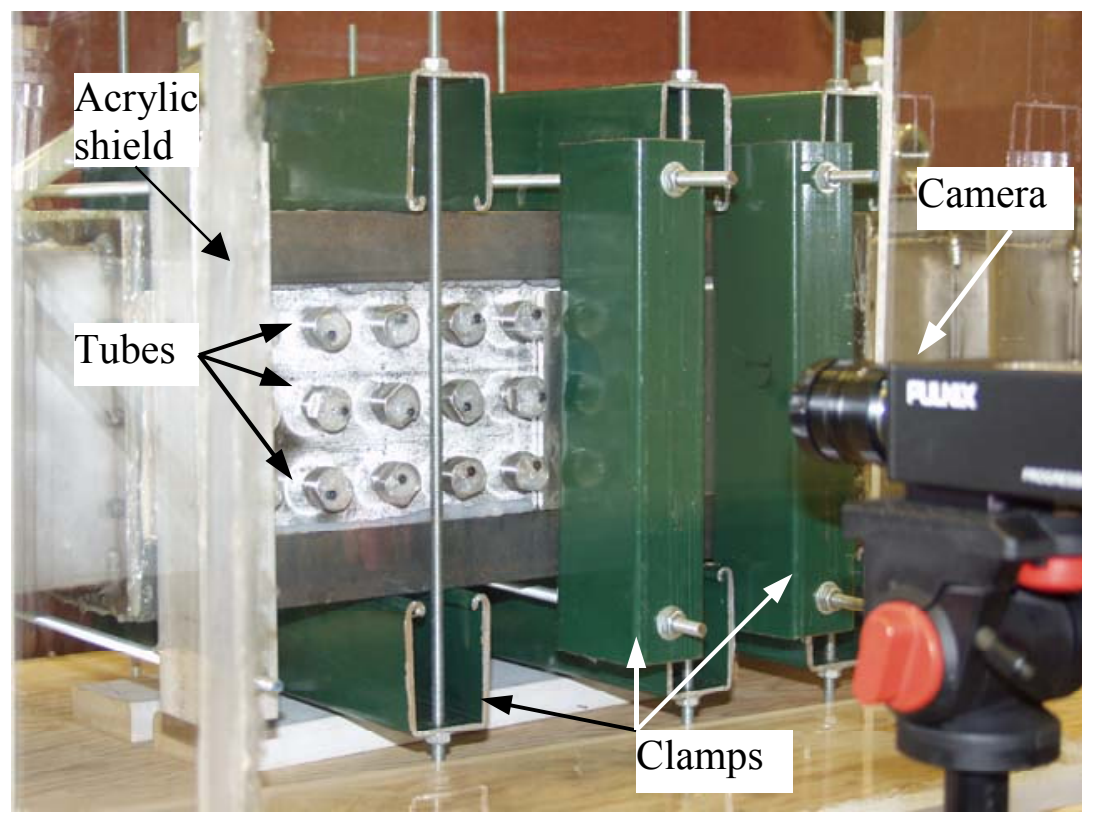

Figure 2.12 Camera set-up.

This array is streamed directly from the limited camera memory bank to a larger intermediate memory module located on the image grabber card. This card is the primary component for data transfer to the computer memory and is critical for determining the speed and size at which images may be acquired in real time. The camera has an independent image grabber card to facilitate and sustain the high capture rate. The image grabber card includes specially customized image capture software for the acquisition and recording of digital images at variable rates. The image grabber card interacts directly with the computer, busing the image data to the computer platform.

The computer support platform is a custom system, specifically designed for this application. A large RAM base allows for long time intervals of high frequency data to be recorded without interruptions due to overflow or image compression. Plenty of system memory is also provided for overhead related to operation of the cameras. The image data is recorded to a 40-gigabyte hard drive from which it may be processed within the acquisition computer or transferred to a dedicated image-processing (data analysis) computer. The data acquisition CPU is sufficiently powerful to handle the logistics of the camera operation as well as handle some of the image-processing load of the data analysis job. 
The specifications of the data acquisition system are provided in Table 2.1, below.

\begin{tabular}{|c|c|}
\hline $\begin{array}{l}\text { Two OmniSpeed LR120 digital video } \\
\text { camers }\end{array}$ & $\begin{array}{ll}\text { - } & 120 \text { frames per second } \\
- & 640 \times 480 \text { pixel resolution } \\
\text { - } & \text { 8-bit (monochrome) pixel depth } \\
\text { - } & \text { High speed digital image grabber }\end{array}$ \\
\hline $\begin{array}{l}\text { Dedicated computer platform for operation } \\
\text { of cameras and real-time data recording. }\end{array}$ & $\begin{array}{ll}\text { - } & \text { Pentium 3,1 GHz processor } \\
\text { dedicated to camera operations } \\
\text { - } & 1.5 \mathrm{~GB} \text { of RAM for high rate } \\
\text { capture of large image sets } \\
\text { - } & \text { 40GB of hard drive space for } \\
\text { temporary storage of images } \\
\text { - } \\
\text { 100baseT Network Interface Card } \\
\text { for fast transfer of data to post- } \\
\text { processing unit }\end{array}$ \\
\hline
\end{tabular}

\subsubsection{Calibration}

Because the reduced velocity depends on the natural frequency of vibration of the tubes, it is essential that the structural parameters governing the tube frequency are carefully calibrated. In this case, the tension on the supporting piano wires is the control parameter. The tubes were assembled one at the time and individually calibrated. The calibration procedure consisted simply of measuring a tube's response to a sudden impulsive force. The impulse was applied to the tube by first displacing the tube and holding it at a distance away from the static equilibrium position. The tube was then released and the elastic reaction force due to the tension of the wire was utilized as the impulse force (i.e. an initial condition of non-zero displacement was used rather than an initial non-zero velocity condition). The motion of the tube was then recorded with the digital camera. The acquisition time was set to 0.5 seconds for a total of 60 images. The number of cycles completed by the tube during the half second, multiplied by two, represented the approximate natural frequency of the tube.

To investigate the influence of such a parameter to the onset of the fluid elastic instability, the tubes were calibrated in air to three different frequencies: 12,16 and 20 $\mathrm{Hz}$, in consideration of the target reduced velocities and based on the tube diameter and expected flow speeds. The tension in the supporting wire was then adjusted and the process was repeated until all tubes were calibrated to the same frequency. In order to check the accuracy of the calibration, a strobe lamp was used in conjunction with the camera. The frequency of the strobe was set at the current calibration frequency and the vibration of each tube was observed in real time, using the camera, under the strobe illumination. The accurately tuned tubes would appear motionless under the strobe lighting since their cycles of vibration were in exact phase with the strobe frequency. 
For the sake of simplifying the measurement of the natural frequencies, the tubes are calibrated in air. Because of the low density of air there are no significant effects due to fluid dynamic coupling between the tubes. Furthermore, for the same reason, boundary effects can be neglected. It is clear, however, that the natural frequency is changed once the tube array is submerged in water. To investigate the effect of the fluid on the natural frequency, an additional test has been conducted in still water, for a single vibrating tube surrounded by fixed tubes, calibrated in air to $16 \mathrm{~Hz}$. The tension applied to the wires of the tube support was the same as the tension applied to the wires of the tube calibrated in air, i.e. the wire tensions used for the two tests were held constant. The natural frequency measured in these conditions was $12.5 \mathrm{~Hz}$. The observed displacements of the tubes in both water and air were plotted on a time basis, and the frequency of vibration has been determined with a Fourier transform of the displacement signal. The surrounding array of tubes had to be held fixed because of the strong fluidforce coupling between the tubes; it was observed that the vibration of the single tube, subject to the initial displacement, induced a significant vibration of the adjacent tubes if left free. The overall effect of this interaction (in still water) was that the single displaced tube would initially vibrate, but would quickly transfer energy through the fluid and induce motion in the adjacent tubes, while it's own amplitude of vibration would damp out over a short period of time.

The tube displacement responses and respective Fourier transforms are reported in the following figures $(2.13$ through 2.20$)$ for the three calibrations in air and the test in still water.

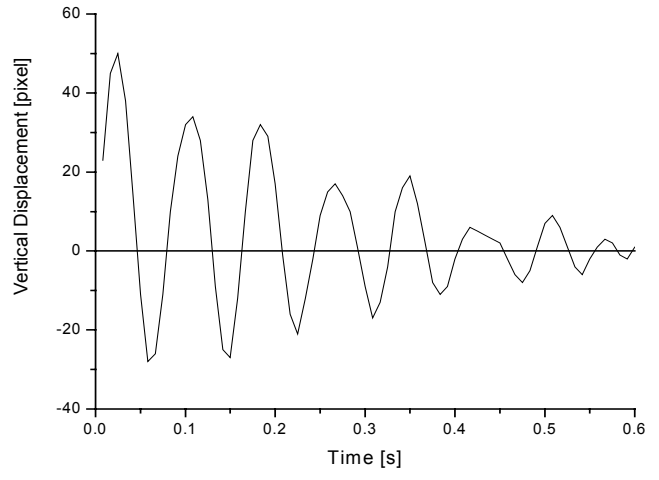

Figure 2.13 Vertical displacement for tube vibrating at $12 \mathrm{~Hz}$ in air.

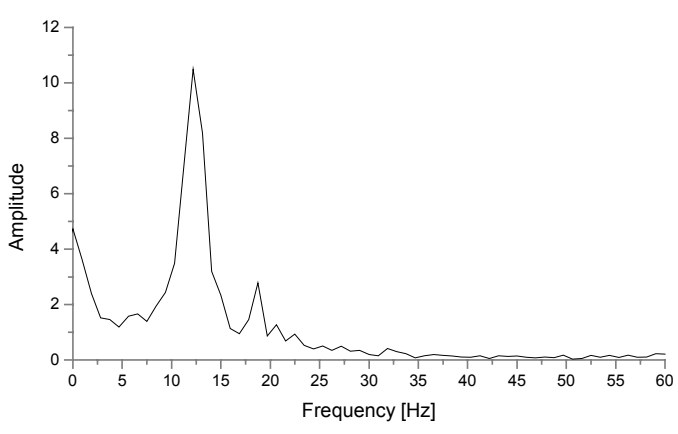

Figure 2.14 Fourier transform for the tube vibrating at $12 \mathrm{~Hz}$ in air. 


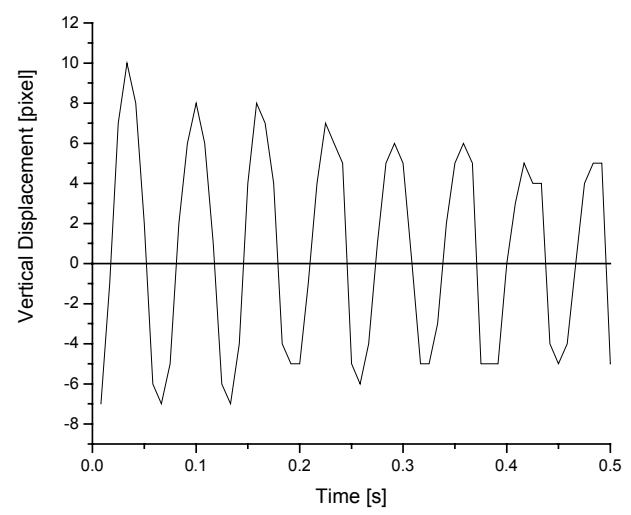

Figure 2.15 Vertical displacement for tube vibrating at $16 \mathrm{~Hz}$ in air.

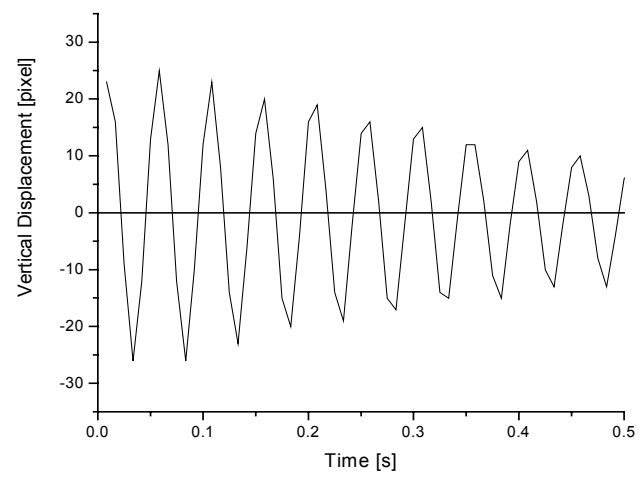

Figure 2.17 Vertical displacement for tube vibrating at $20 \mathrm{~Hz}$ in air.

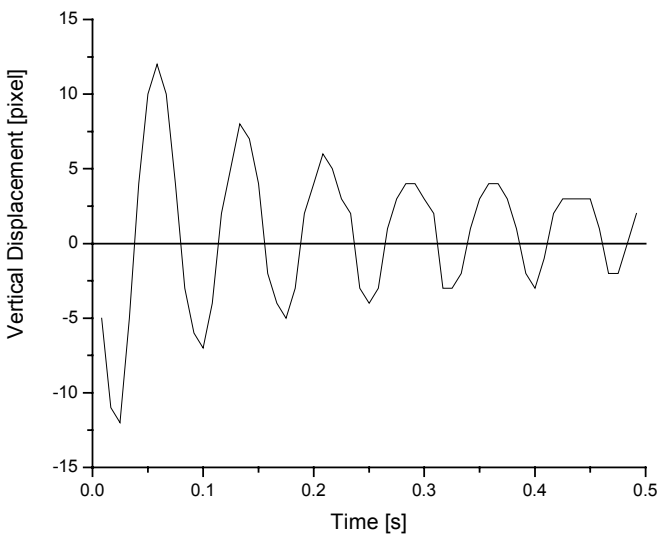

Figure 2.19 Vertical displacement for tube vibrating at $12.5 \mathrm{~Hz}$ in still water.

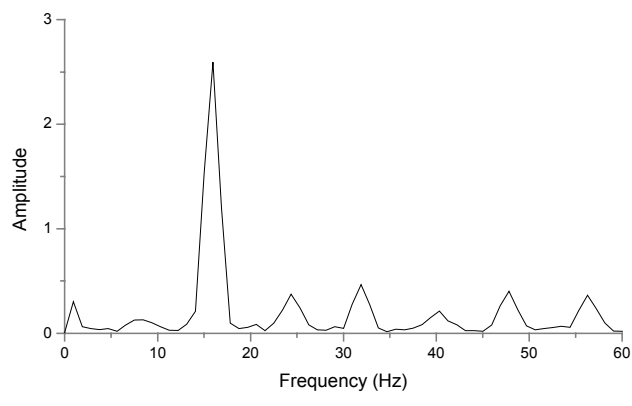

Figure 2.16 Fourier transform for the tube vibrating at $16 \mathrm{~Hz}$ in air.

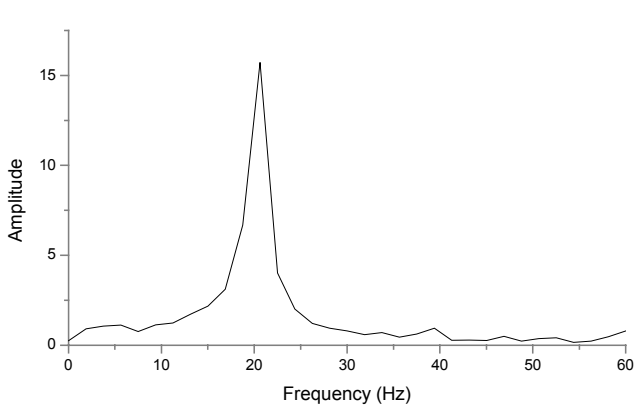

Figure 2.18 Fourier transform for the tube vibrating at $20 \mathrm{~Hz}$ in air.

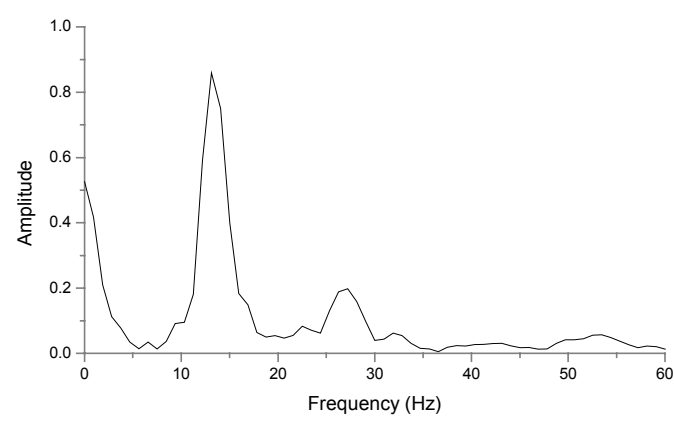

Figure 2.20 Fourier transform for the tube vibrating at $12.5 \mathrm{~Hz}$ in still water.

The procedure showed above consent the calibration of the tubes with good accuracy: the natural frequency of the tubes as computed using the Fourier transform is always in a range of $1 \%$ of the desired frequency. 


\subsection{Two Phase Experiment}

\subsubsection{Flow Loop}

A schematic of the flow loop and the test section is shown in Figure 2.21. The experimental flow loop is designed to pump water from a reservoir having a capacity of 400 gallons. Steam or air can also be introduced into the loop to allow the generation of the two-phase mixture. A 10 horse power centrifugal pump is used to pump the water through the test section. The main flow loop is constructed from 3 inch stainless steel piping and contains a number of flow control and flow bypass valves to control the water flow rate through the test section After passing through the test section, the water or twophase mixture, as the case may be, is returned back to the reservoir tank and recirculated during an experimental run. In the case of the steam-water flow cases, a copper coolant coil in the reservoir is used to reduce the temperature of the steam-water mixture to that of saturated water at atmospheric pressure in the tank. A throttle valve installed at the exit of the test section can be used to pressurize the test section when needed for certain steam-water flow cases.

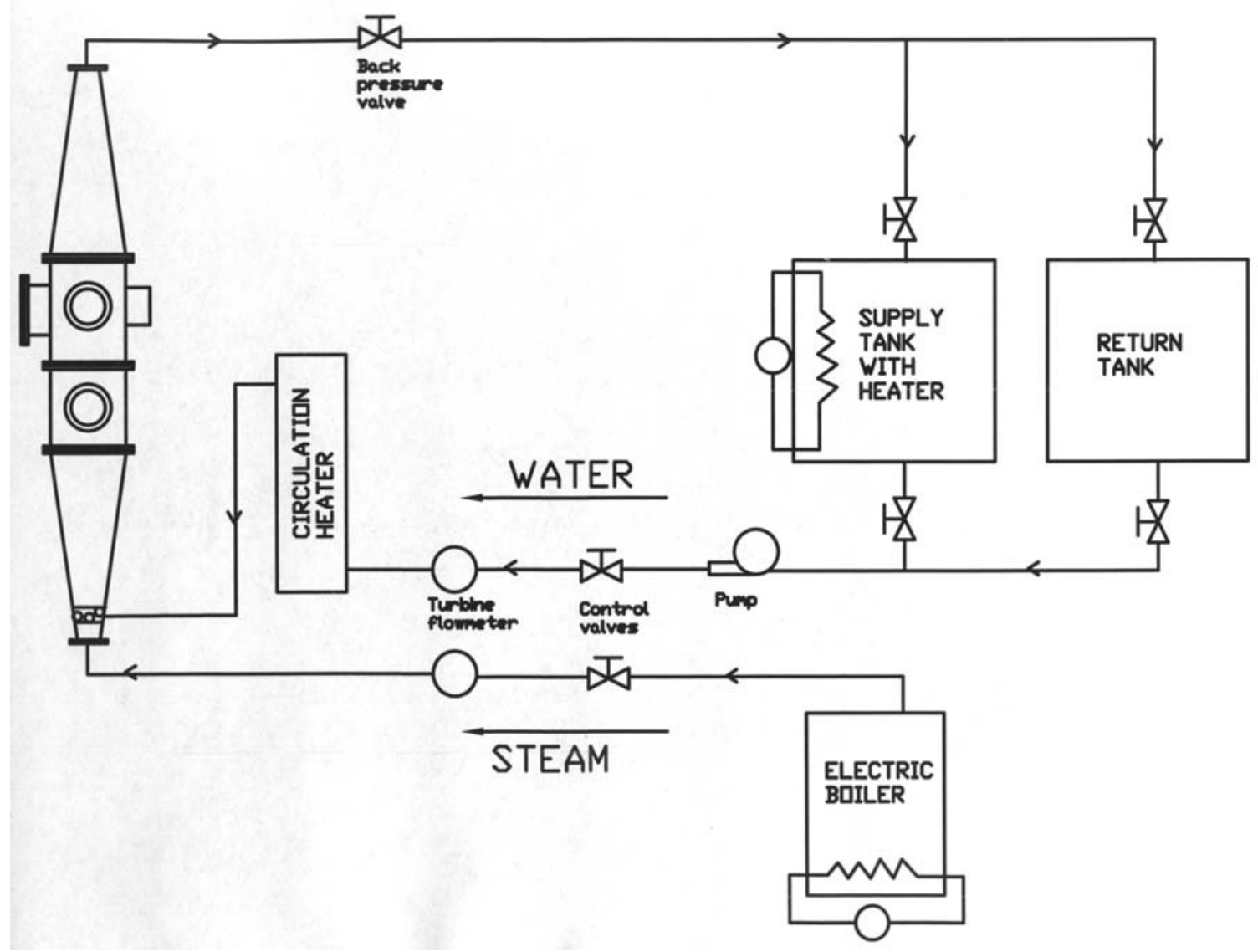

Figure 2.21: Schematic of flow loop for fluid-elastic instability experiments 
A $215 \mathrm{~kW}$ inline circulation heater is provided in the flow loop upstream of the test section to heat the water to saturation for the steam-water flow cases and the elevated temperature cases. With the installed pump, 80 gallons per minute $\left(0.005 \mathrm{~m}^{3} / \mathrm{s}\right)$ of water at $25^{\circ} \mathrm{C}$ can be circulated through the test section. A turbine flow meter is used to measure the water flow rate.

Steam or air is introduced into the test section through a separate flow line. This line is constructed from 1" stainless steel pipe. A turbine flow meter is used to measure the air or steam flow rate in actual cubic feet per minute (acfm). The flow rate is controlled using a combination of globe valve and a gate valve. Utility air from the laboratory air supply line is introduced into the flow line to generate an air-water mixture. A $46 \mathrm{~kW}$ electric boiler that can generate steam at pressures up to $100 \mathrm{psi}$ generates steam. An energy balance on the boiler shows that it can generate about 25 cubic feet per minute of steam at atmospheric pressure, and about 4 cubic feet per minute of steam at a gage pressure of 100 psi.

During experimental runs, the steam needs to be generated at higher pressures than the test section pressure to overcome the pressure drop at the mixing section. Thus, a part of the steam condenses on mixing with the saturated water. Thermocouples (K type, Omega Engineering) and pressure transducers (PX-202 100GV and ...) measure the temperature and pressure just downstream of the gas flow meter, at the inlet of the mixing section on the air/seam line, downstream of the test tube bundle and upstream of the test tube bundle. Table 2.2 gives a summary of the important operating parameters achievable with the installed flow loop.

Table 2.2: Maximum achievable operating parameters

\begin{tabular}{|l|l|}
\hline \multicolumn{1}{|c|}{ Parameter } & \multicolumn{1}{c|}{ Maximum achievable value } \\
\hline Water flow rate (measured) & $80 \mathrm{gpm} @ 25^{\circ} \mathrm{C}$ \\
\hline Air flow rate (measured) & $18 \mathrm{acfm} @ 101 \mathrm{kPa}$ \\
\hline Steam flow rate (calculated) & $25 \mathrm{acfm} @ 101 \mathrm{kPa}$ \\
\cline { 2 - 2 } & $4 \mathrm{acfm} @ 790 \mathrm{kPa}$ \\
\hline Void fraction: Air-water flow (measured) & $0-50 \%$ \\
\hline $\begin{array}{l}\text { Void fraction: Steam-water flow } \\
\text { (calculated) }\end{array}$ & $0-40 \%$ \\
\hline Pressure (max. design pressure) & $100 \mathrm{psig}$ \\
\hline Temperature (max. design temperature) & $450^{\circ} \mathrm{C}$ \\
\hline
\end{tabular}




\subsubsection{Test section}

The test section consists of four sub-assemblies: (1) mixing section, (2) viewing section, (3) test section, (4) exit section. A schematic of each of the sections is shown in Figure 2.22. The test section features an all-stainless-steel construction to withstand the high temperature and corrosion from use with steam-water mixture.

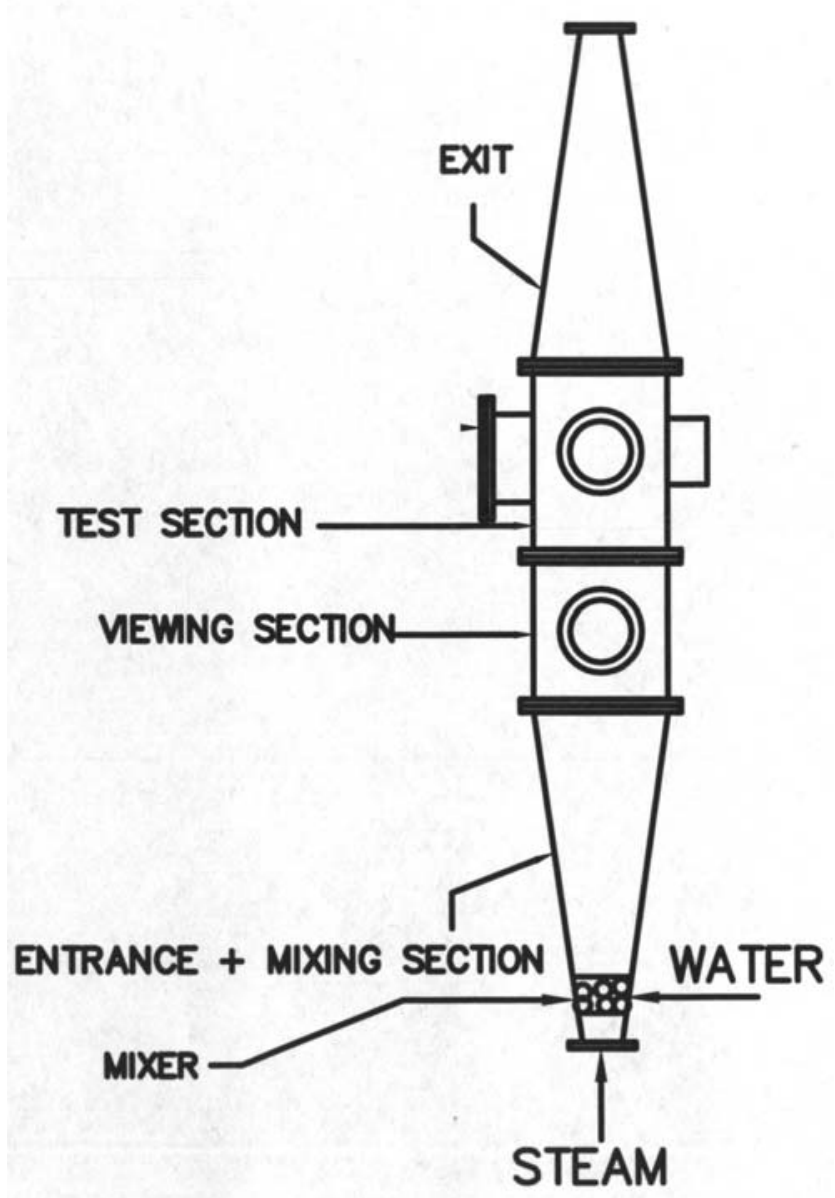

Figure 2.22: Schematic of test section assembly

The mixing section ensures that the water and air/steam mix in a proper manner to provide a more or less uniform two-phase flow. It connects to the water line at the exit of the circulation heater through a nozzle plate three inches in diameter and consisting of a symmetric arrangement of 0.125 " diameter holes. The nozzle plate is used to break the incoming water into smaller jets for uniform mixing. The steam/air line is also connected to the bottom of the mixing section. The steam/air and water jets mix over a packed bed of 1 " stainless steel spheres stacked $0.1 \mathrm{~m}$ high. Honeycombs are provided after the packed bed to straighten the flow. The mixing section also acts as a diffuser to provide a smooth transition from the circular cross section of the pipe to the rectangular cross section of the remaining test section. The length of the mixing section is $0.62 \mathrm{~m}(24.25$ inches). 
The viewing section has a rectangular cross section of $0.23 \mathrm{~m} \times 0.09 \mathrm{~m}$ (9 inches $\times 3.5$ inches $)$ and is $0.25 \mathrm{~m}$ (10 inches) high. It is equipped with two glass view ports, $10.16 \mathrm{~cm}$ (4 inches) in diameter, on either side through which free stream void fraction measurements are made. This section along with the mixing section also allows the twophase flow to develop before reaching the test section.

The test section houses the rod bundle. It has a cross section of $0.24 \mathrm{~m} \times 0.09 \mathrm{~m}$ ( 9.5 inches $\times 3.5$ inches) and is $0.33 \mathrm{~m}$ (13 inches) in height. It houses a fixed tube array that is located immediately upstream of the region housing the fully flexible array where the fluidelastic instability measurements are made. The dummy tubes are provided for flow development over an array consisting of 5 rows of fixed cylinders. The flexible tube array is suspended from the inner walls of the test section using stainless steel piano wires. Details of the tube arrays are provided in the next section. On three sides of the test section glass view ports are provided for visualization of the flow and the tubes as they vibrate. Half cylinders are attached to the inner walls of the test section to provide a uniform boundary condition at the walls of the test section.

The exit section has the same dimensions as that of the mixing section and provides a smooth transition from the rectangular to circular cross section at the exit. The entire assembly is mounted on a robust support structure. Vibration isolation rubber pads are used at the various joints to isolate vibrations of the frame, which accompany the twophase flow, from those of the test section and tube array. Figure 2.23 shows photographs of the test section assembly and the entire flow loop.

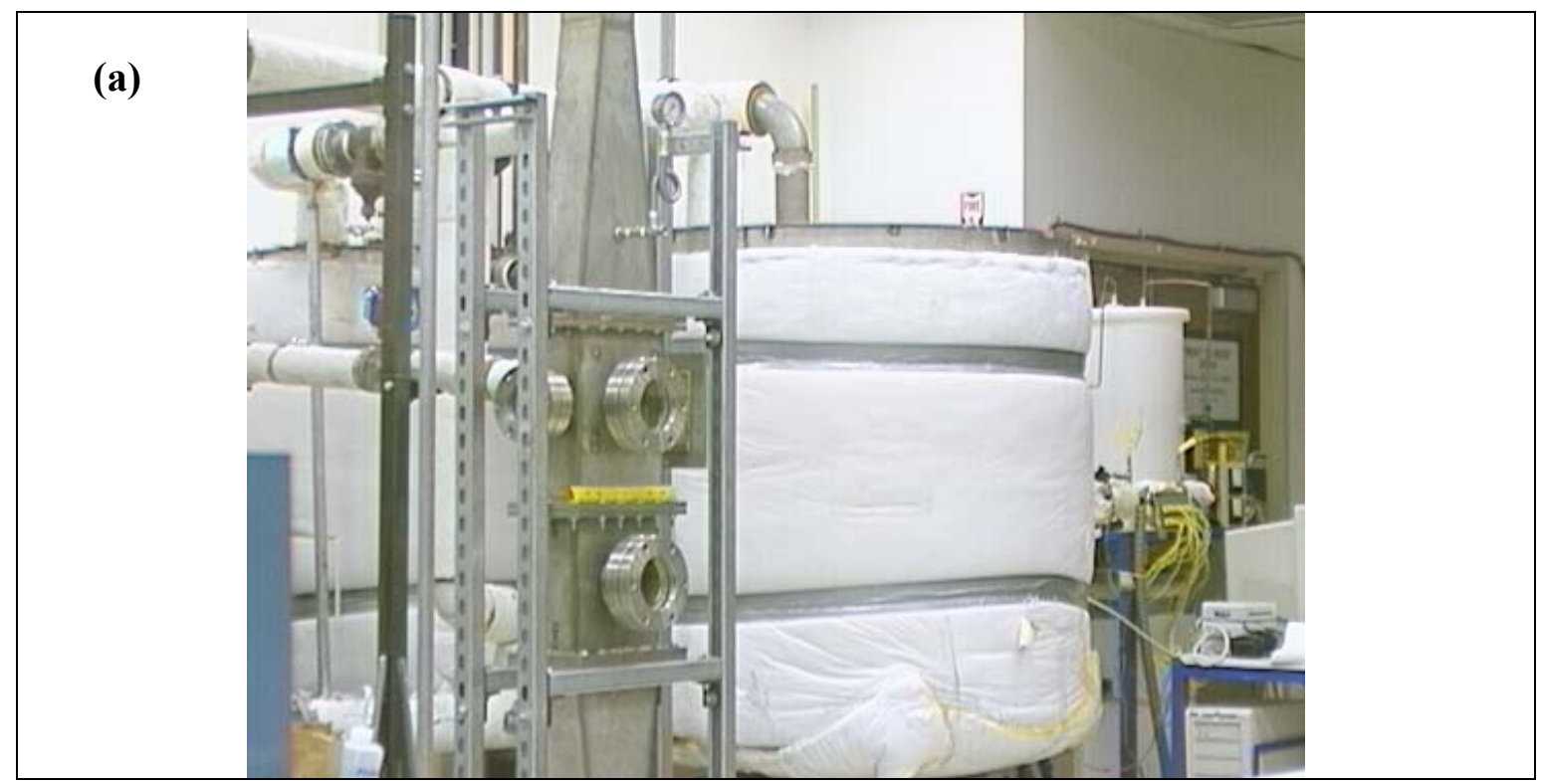




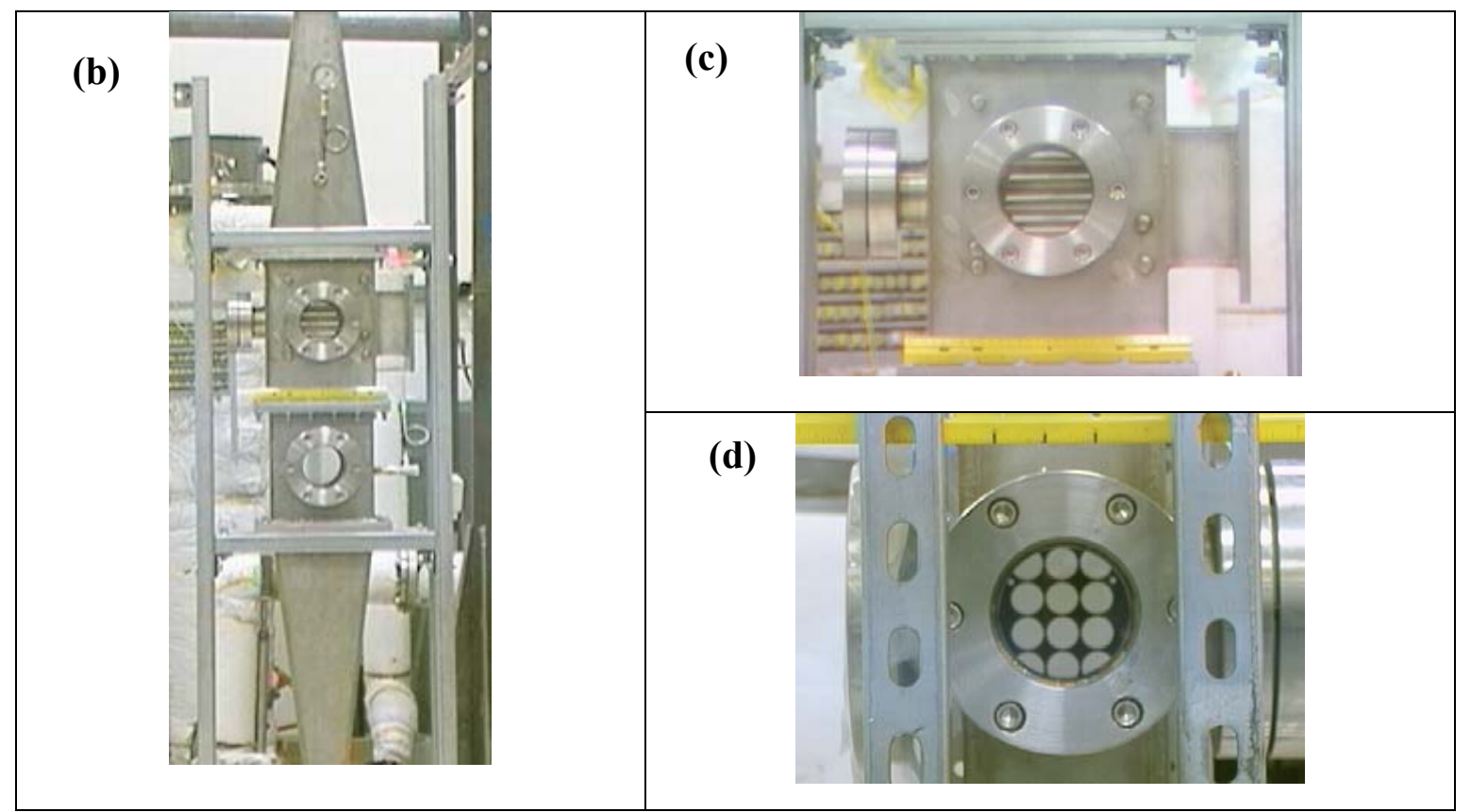

Figure 2.23: Photographs of flow loop and test section

(a) Flow loop, (b) Test section assembly, (c) Test section (Half cylinders can be seen), (d) Test section (End view)

\subsubsection{Tube-array}

In order to simulate heat exchanger tubes, an array of stainless steel tubes are used. In practical heat exchangers, these tube arrays are arranged in a number of geometric patterns, such as normal square array, rotated square array, normal triangular array, rotated triangular array and a staggered array. Since the most commonly used patterns are the normal square array and the rotated square array, both these patterns are planned to be tested in the experiments. The schematic of these arrays are shown in Figure 2.24 below:
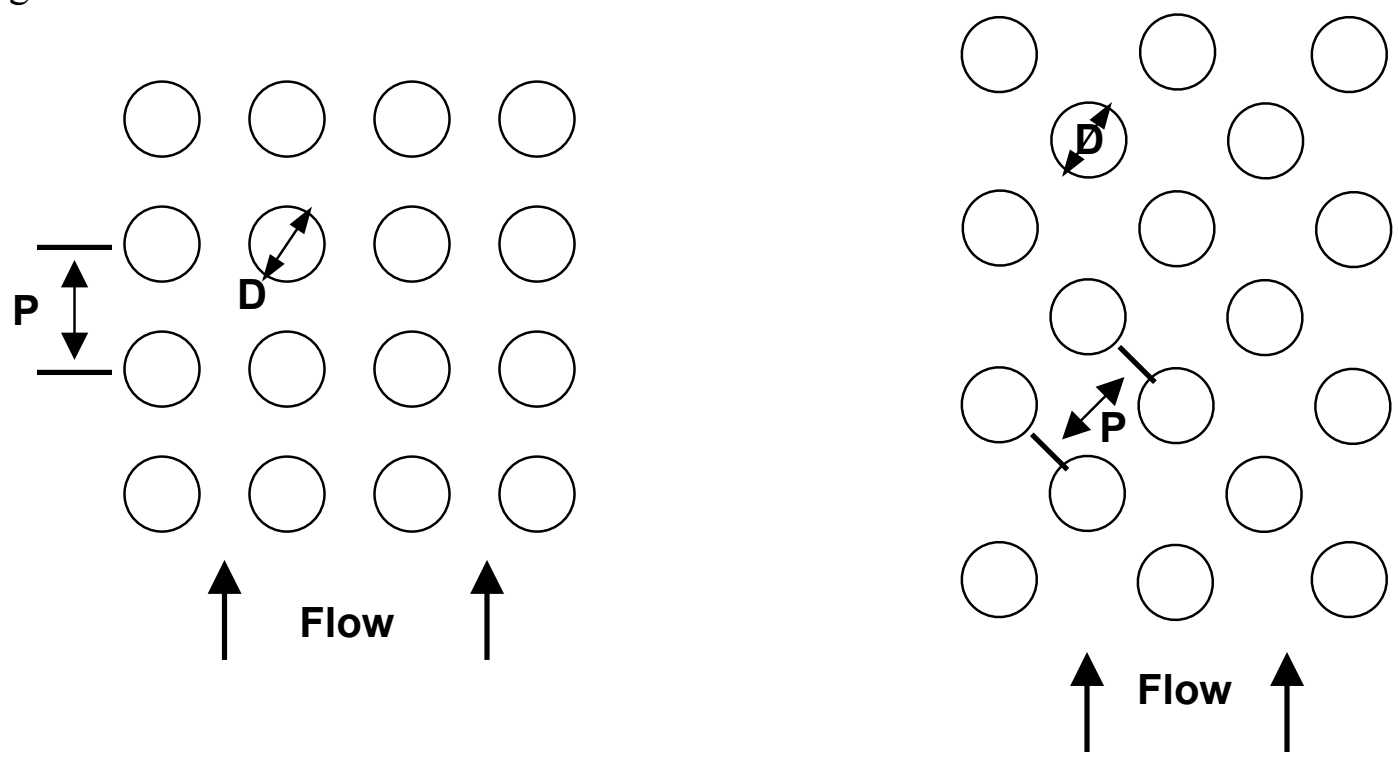

Figure 2.24: Tube-array patterns (a) Normal square, (b) Rotated square 
In the case of a square array, the longitudinal pitch and the transverse pitch are the same. However, they may be different as in the case of a staggered array. The present experiments to study fluid-elastic instability are carried out on a $5 \times 3$ array of flexible tubes. Upstream of the flexible array, a $5 \times 3$ dummy array of fixed tubes is provided for flow development. The stainless steel tubes are $0.2 \mathrm{~m}$ ( 8 inches) in length, have an outer diameter of $0.015 \mathrm{~m}$ (0.625 inches), and an inner diameter of $0.014 \mathrm{~m}(0.555$ inches $)$. The tubes are sealed off at the end using end caps that also serve as connection points for stainless steel piano wires. The mass of each tube including the end caps is $0.13 \mathrm{~kg}$. The pitch-to-diameter $(P / D)$ ratio for the normal square array is chosen to be 1.4. This is selected to minimize the possibility of occurrence of vortex shedding instability during the experiments.

Stainless steel piano wires ( 0.020 inch diameter) are used in the flexible array to allow for tube vibration at easily realizable and measurable frequencies. In addition, piano wires allow the tubes to vibrate in both the lift and drag directions simultaneously. The length of the piano wire between the tube and the ends of the test section is $0.03 \mathrm{~m}$ (1.1875 inches). On one end, the piano wires attach to a stainless steel grid structure. On the other end, they terminate in a tightening mechanism that is used to tighten or loosen the piano wires hence changing the natural frequency of the tubes. The tensioning mechanism consists of a nut that can be traversed across a threaded lead screw to tighten or loosen the piano wire and hence alter the tension of the tube-wire system. The schematic of the tensioning mechanism is shown in Figure 2.25. It is to be noted that the tensioning mechanism only allows for fine-tuning of the natural frequency so that all tubes in the array can be tuned to approximately the same natural frequency.

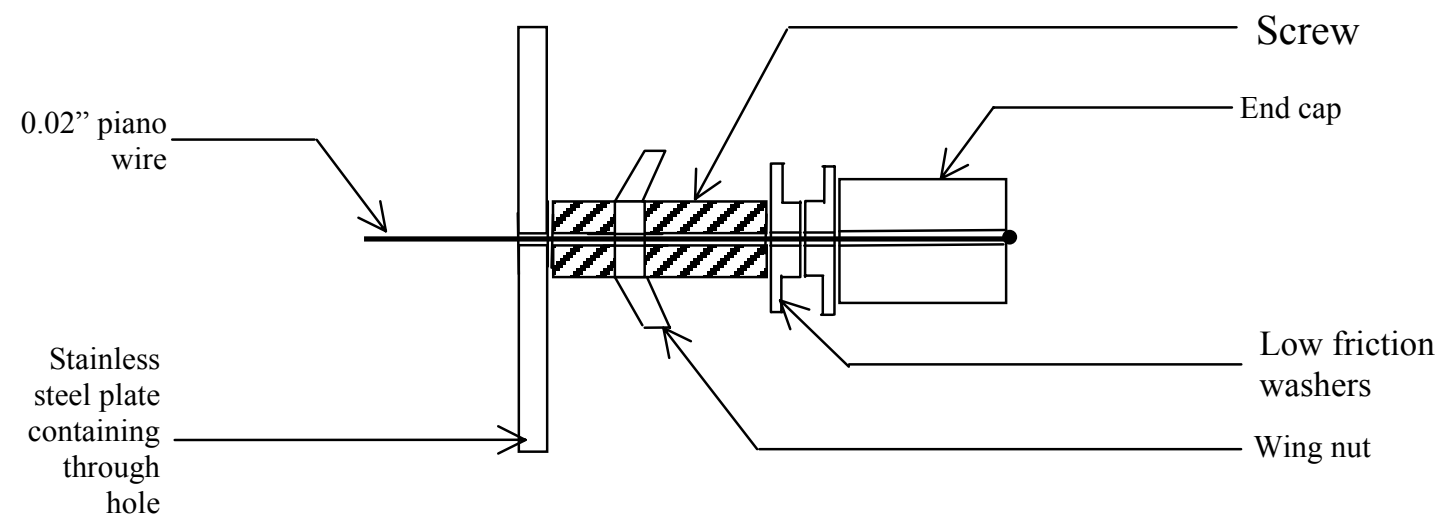

Figure 2.25: Tensioning mechanism 


\subsubsection{Measurement and Data-Acquisition System}

Resistance strain gages are used to measure the tube vibration. The strain gages respond to change in applied strain and hence can be used to give an indication of the tube vibration, which is characterized by the dynamic change in strain in the piano wire. This approach has been used by a number of researchers in recording flow-induced vibrations. The advantages of using strain gages are their small size, mass and sensitivity. On the other hand, making them survive the harsh environment of steam-water flow proves to be a great challenge.

In the present setup, the piano wires are the only components that undergo change in strain with tube vibration. Hence, the strain gages need to be bonded on to the piano wires. Strain gages from Measurements Group (CEA-06-125-350UN) are used for this purpose. This series of strain gages has a nominal resistance of $350 \Omega$. Two strain gages bonded on opposite sides of the piano wire are connected in a half bridge configuration. This output from the strain gages input is fed to a bridge completion module (Omega, BCM-1) that outputs a milli volt signal that is then detected by the data acquisition system. This configuration was used by Joo and Dhir $(1994,1995)$ to measure the vibrations in their cantilever-tube system and was found to yield a better signal than a quarter bridge configuration consisting of one strain gage. In addition, two strain gages also allow for compensation due to thermal effects and change in length of the piano wire. Stainless steel shims (0.004 inches thick) spot-welded to the piano wire are used as surfaces for bonding the strain gages. The thickness of the shims is chosen such that the addition of the shim minimally alters the stiffness of the piano wire. The schematic of the arrangement of the shims with respect to the piano wires is shown in the Figure 2.26.

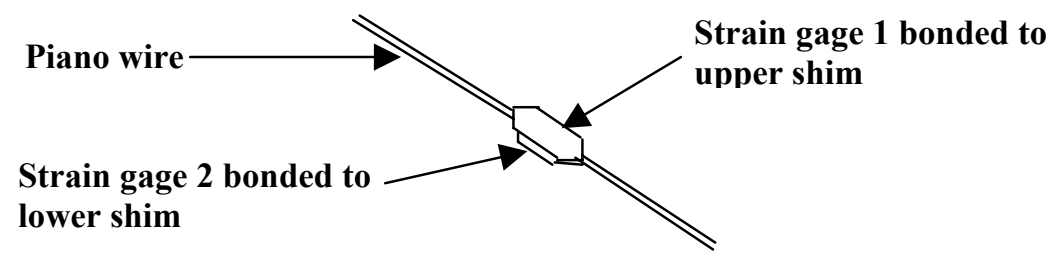

Figure 2.26: Bonding of strain gages to shims

Electronic data acquisition is performed using a data acquisition board (National Instruments, AT-MIO-64-E3). The strain gage signals are sampled at $200 \mathrm{~Hz}$ in order to measure the vibration. The various temperatures, pressures and flow rates are also recorded at $200 \mathrm{~Hz}$ using the same data acquisition system. 


\subsubsection{Measurement Methodology}

This section describes the experimental methods and techniques using which the experimental parameters are determined during the instability runs. These include the frequency of vibration, the damping ratio, the tube displacement and the void fraction of the two-phase mixture.

\subsubsection{Flow velocity measurement}

Superficial fluid velocity is calculated from the volume flow rates measured using the turbine flow meters. For the single phase case, the free-stream velocity is calculated using the formula

$$
V_{\infty}=\frac{Q_{l}}{A}
$$

where $V_{\infty}$ is the freestream velocity, $Q_{l}$ is the liquid volume flow rate, and $A$ is the cross section area upstream of the tube array. The pitch velocity is calculated as

$$
V_{p}=\frac{(P / D)}{(P / D-1)} V_{\infty}
$$

where $V_{p}$ is the pitch velocity and $P / D$ is the pitch-to-diameter ratio. For two-phase flow, the flow velocity is calculated from the mass flow rates of the individual phases using the formula

$$
V_{2 \phi, \infty}=\frac{(n \&+n k g)}{A \cdot \rho_{2 \phi}}
$$

where $\rho_{2 \phi}$ and $V_{2 \phi}$ are the two-phase density and velocity, and $n \&$ and $n \&_{g}$ are the mass flow rates of the liquid and gas phases. The two-phase equivalent density is calculated using the formula

$$
\rho_{2 \phi}=\alpha \cdot \rho_{g}+(1-\alpha) \cdot \rho_{l}
$$

where $\rho_{l}$ and $\rho_{g}$ are the densities of the liquid and gas phases, and $\alpha$ is the measured void fraction.

\subsubsection{Frequency measurement}

The frequency of vibration of the tubes is measured using two different methods: (1) time history of strain gage signal, (2) Fourier transform of strain gage signal. The first method is used in conjunction with the logarithmic decrement method for calculating the damping ratio when the tubes are immersed in stationary fluid (frequency and damping in single phase fluid with no flow). In this method, the frequency is calculated by observing the number of cycles in a particular time interval of the signal. The Fourier transform on the other hand provides a frequency domain representation of the signal and the dominant frequencies of the system appear as peaks in the amplitude spectrum. Hence, the natural frequency can be easily determined by observing the peak of the Fourier transform. Hence, this method is used for the fluid-elastic instability experiments with single and two-phase flow. 


\subsubsection{Damping ratio measurement}

System damping is the result of energy dissipation during vibration and is one of the most important factors affecting fluid-elastic instability. Three factors contribute to the total damping of a structure: (1) fluid damping due to fluid drag, viscous dissipation, and radiation to surrounding fluid, (2) internal material damping due to losses within the medium of the structure, (3) "structural damping" mainly due to friction, impact, scrapping, and motion of trapped fluid. Additionally, research has shown that in twophase flow conditions, a two-phase damping component is also present. The damping in a structure is characterized by its damping ratio $\zeta$. Mathematically, the damping ratio is a function of the ratio of the energy dissipated by the structure due to damping to the total stored energy.

The damping of tubes in an array can be measured in two ways: (1) the free-decay or logarithmic decrement method, (2) Half-power bandwidth method. The free-decay or logarithmic decrement method involves the time-history of the strain gage signal and the damping ratio is calculated by observing the decay of the signal amplitude. The following example illustrates this method.

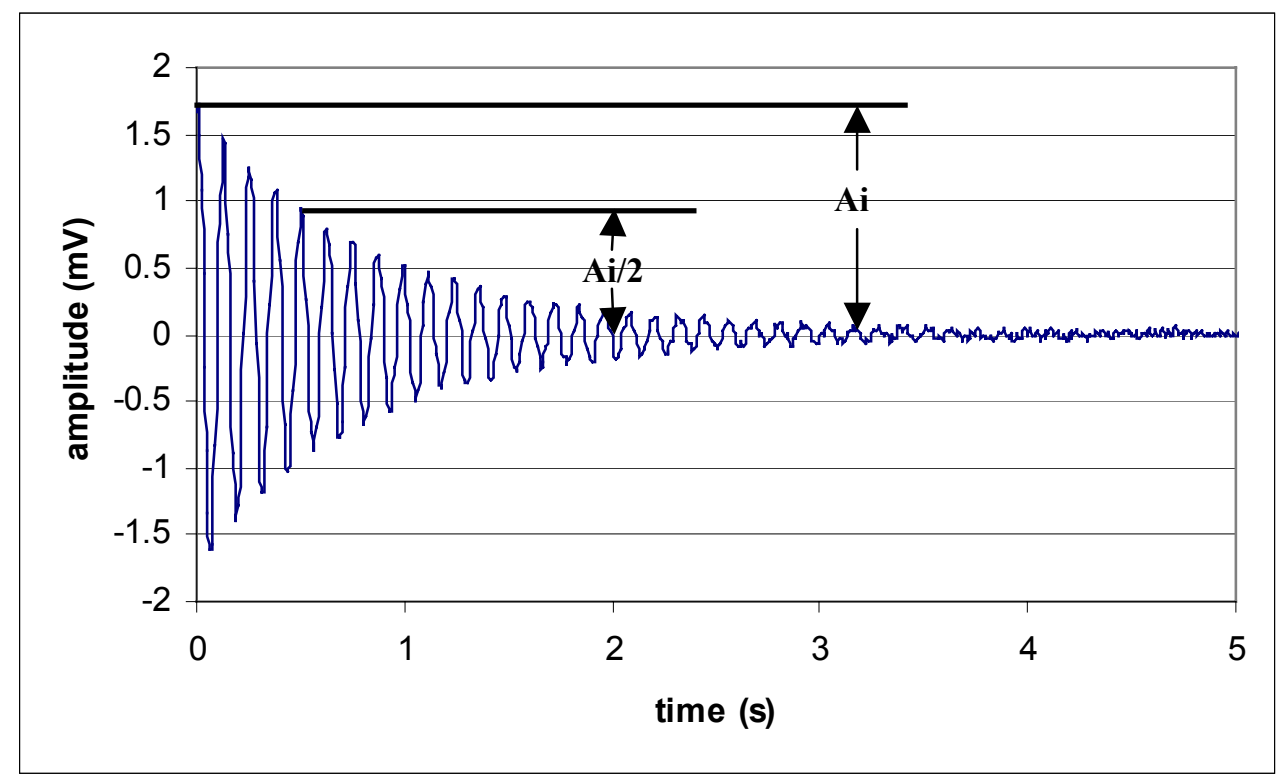

Figure 2.27: Time decay of tube vibration (Cantilever tube system)

From a sample signal, the number of cycles $(N)$ required for the amplitude of the signal to decay to half its initial value is noted. Once $N$ is determined, the damping ratio is calculated as $\zeta=\frac{\log 2}{2 \pi N}$. The disadvantage of this method is that in most cases, $N$ can only be approximated as an integer. Since this method is based on an exponential decay of the signal, an equivalent and more accurate technique is to fit the points of maxima of the signal amplitude with an exponential curve. Once the exponential is fitted and its equation obtained as $y=C e^{-B x}$, the damping ratio can be equivalently calculated as 
$\zeta=\frac{-B}{2 \pi f_{n}}$. This technique gives reliable results in a free-decay situation but the uncertainty in the measurement is largely affected by the accuracy of the exponential curve fit. The half-power bandwidth method is based on the Fourier transform of the strain gage signal. This is illustrated using an example as follows. Figure 2.28 shows the amplitude spectrum of the signal in Figure 2.27.

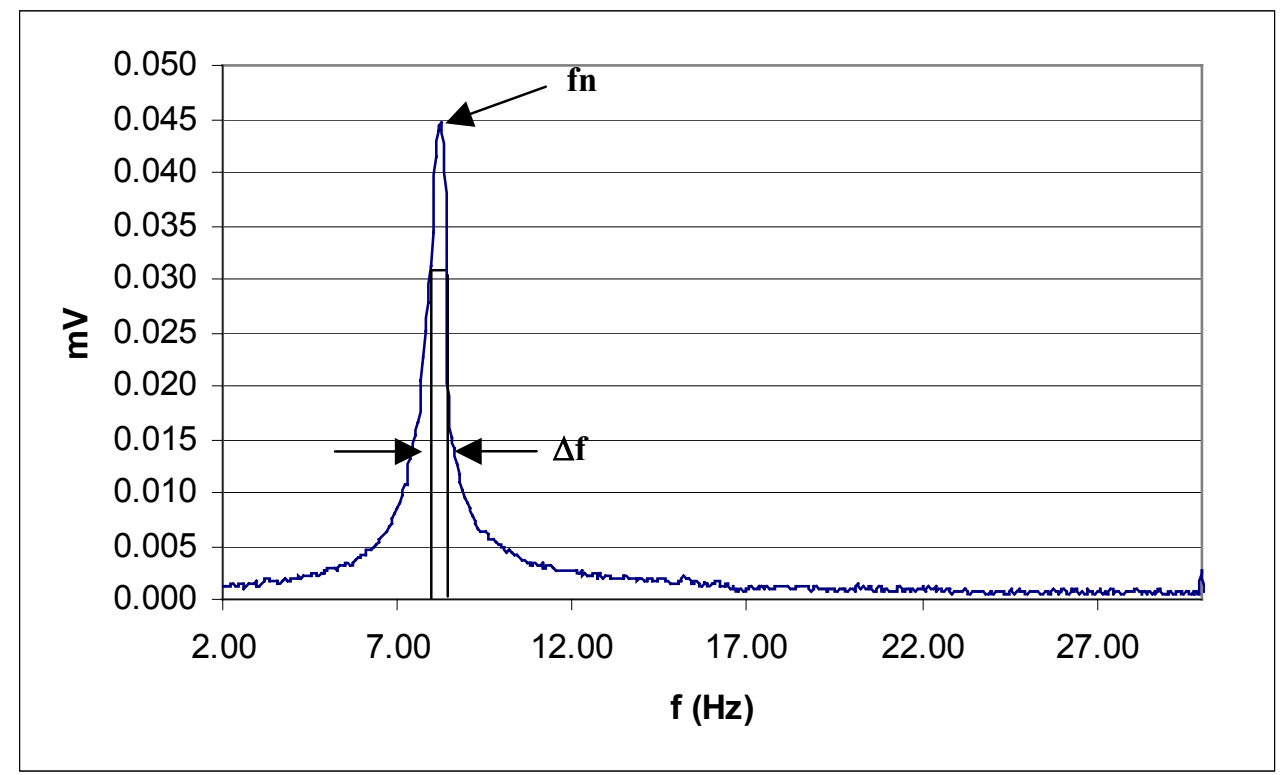

Figure 2.28: Amplitude spectrum of cantilever-tube system strain gage signal

From the amplitude spectrum, the damping ratio is computed using the formula

$$
\zeta=\frac{\Delta f}{2 f_{n}}
$$

where $\Delta f=$ frequency band at 0.707 times the peak amplitude. However, the decay around the peak of the signal is not often smooth and may lead to erroneous results. Sometimes, the curve fit method suggested by Pettigrew et al. may be employed to fit a smooth curve to randomly selected points along the spectrum. The data points are fitted according to the equation

$$
\frac{A_{y} k}{F_{O}}=\left\{\left[1-\left(\frac{f}{f_{n}}\right)^{2}\right]^{2}+4 \zeta^{2}\left(\frac{f}{f_{n}}\right)^{2}\right\}^{-1 / 2}
$$

which describes the amplitude response in the frequency domain of a single degree-offreedom structure (Ref. Blevins, pp. 317) where $A_{y}$ is the amplitude of the response, $k$ is the stiffness of the system, and $F_{O}$ is the amplitude of the forcing function. In the present study, the curve fit method is found to under predict the damping ratio. Hence, the halfpower bandwidth method is used to determine damping ratio in all the two-phase flow experiments. 


\subsubsection{Tube displacement}

The initial plan for measuring the tube displacement was to calibrate the strain gage signal to the actual displacement of the tube using images obtained using a highspeed camera. The sampling rates of the camera and the strain gage data acquisition system were synchronized and the waveforms compared to obtain the calibration in terms of millimeter per milli volt. While this system yielded excellent agreement in the cases where no flow was involved, the drift of the strain gage signals with temperature and flow velocity limited the application of this technique to two-phase flow. Hence, in order to measure the displacement in the two-phase flow runs, specific tests were carried out with a high speed camera recording the actual movement of the test tube from which the tube displacement was measured using image processing techniques.

\subsubsection{Void fraction}

The void fraction of the two-phase mixture in the free-stream is measured using a dual-beam gamma-densitometer. The gamma densitometer consists of two sources: Cesium-137 and Americium-241, and a detector made of NaI. The gamma beam emitted by the source is collimated by passing through a circular slit $(2.54 \mathrm{~cm}$ diameter) before entering the test section. On the opposite end, the attenuated beam is collimated once again before being incident on the detector. The detector produces electronic pulses that are then transmitted to a 1024 channel analyzer. The analyzer displays the number of pulses versus the energy level. The integral under this curve $(I)$ is recorded for each medium -liquid $\left(I_{l}\right)$, gas $\left(I_{g}\right)$, and two-phase mixture $\left(I_{\alpha}\right)$ for a specified period of time. The void fraction is then calculated using the formula

$$
\alpha=\frac{\ln \left(I_{\alpha}\right)-\ln \left(I_{l}\right)}{\ln \left(I_{g}\right)-\ln \left(I_{l}\right)}
$$

The values of the void fraction will be used to calculate the effective density of the twophase mixture as described previously.

Table 2.3 summarizes the methods used to measure the experimental parameters during the fluid-elastic instability experimental runs. 
Table 2.3: Summary of experimental methods for calculating fluid-elastic instability parameters

\begin{tabular}{|l|l|}
\hline \multicolumn{1}{|c|}{ Parameter } & \multicolumn{1}{|c|}{ Experimental Method } \\
\hline Frequency of vibration & $\begin{array}{l}\text { Amplitude spectrum of the strain-gage } \\
\text { signal }\end{array}$ \\
\hline Damping ratio & $\begin{array}{l}\text { Amplitude spectrum using bandwidth } \\
\text { method }\end{array}$ \\
\hline Tube displacement & High speed movies of tube vibration \\
\hline Void fraction & $\begin{array}{l}\text { Radiation attenuation method using } \\
\text { gamma-densitometer }\end{array}$ \\
\hline Fluid density & $\begin{array}{l}\text { Effective density calculated from measured } \\
\text { void fraction }\end{array}$ \\
\hline Flow velocity & $\begin{array}{l}\text { Mass flow rates of liquid and vapor/air } \\
\text { using turbine flow meter; effective density } \\
\text { of mixture and flow area }\end{array}$ \\
\hline Tube mass & $\begin{array}{l}\text { Tube mass measurements and analytical } \\
\text { expression for hydrodynamic mass }\end{array}$ \\
\hline
\end{tabular}




\section{Fluid-elastic Instability Experiments}

\subsection{Single Phase Experiment}

\subsubsection{Data Processing Methods}

\subsubsection{Image processing}

Once the image data has been saved onto the acquisition computer hard drive, the data must be processed in order to extract the desired tube displacement information. Part of the data is transferred to a second computer platform (analysis computer) dedicated specifically for image processing, while the other part remains on the acquisition computer for processing or temporary storage. The image transfer is accomplished via a local Ethernet (100/10 Base T) connection between the two computers.

The analysis computer acts as the primary computational system. It is responsible for both the majority of the image processing and all analysis of the resulting information. Splitting the time intensive job of processing the images between the two computers significantly reduces the time needed to obtain results for the displacement information. The programs for the image processing are developed using different environments: Adobe Photoshop, Matlab and Java Image.

The initial step in the processing of the data is done by a set of instructions executed as a subroutine by Adobe Photoshop. The raw acquired images of the full vibrating tube array are opened and viewed using Photoshop. These images are relatively large, 8-bit files that contain a significant amount of background or unnecessary image information. For this reason, a rectangular region of interest (ROI) around a single tube is identified and extracted from the image, limiting the size of the image to the relevant data. The ROI tube image is processed to further enhance the tube profile and thus simplify the task of locating the tube center coordinates. First, an edge detection filter is used on the image, which highlights the circular outside and inside edges of the tube profile. Second, the image is thresheld at a pre-set level to eliminate any low intensity noise. The resulting image is then saved as a new image file in a separate directory specific to the particular tube being analyzed. This subroutine, i.e. the image extraction and pre-processing (before the actual analysis), has been automated and can be run as a batch process once a tube ROI within a particular data series has been identified. The method can be applied to each of the visible vibrating tubes within each of the full raw images from the different time series.

The result is a set of smaller binary images containing the profile of the respective tube of interest, which may be analyzed very quickly due to the simplified form of the information. 


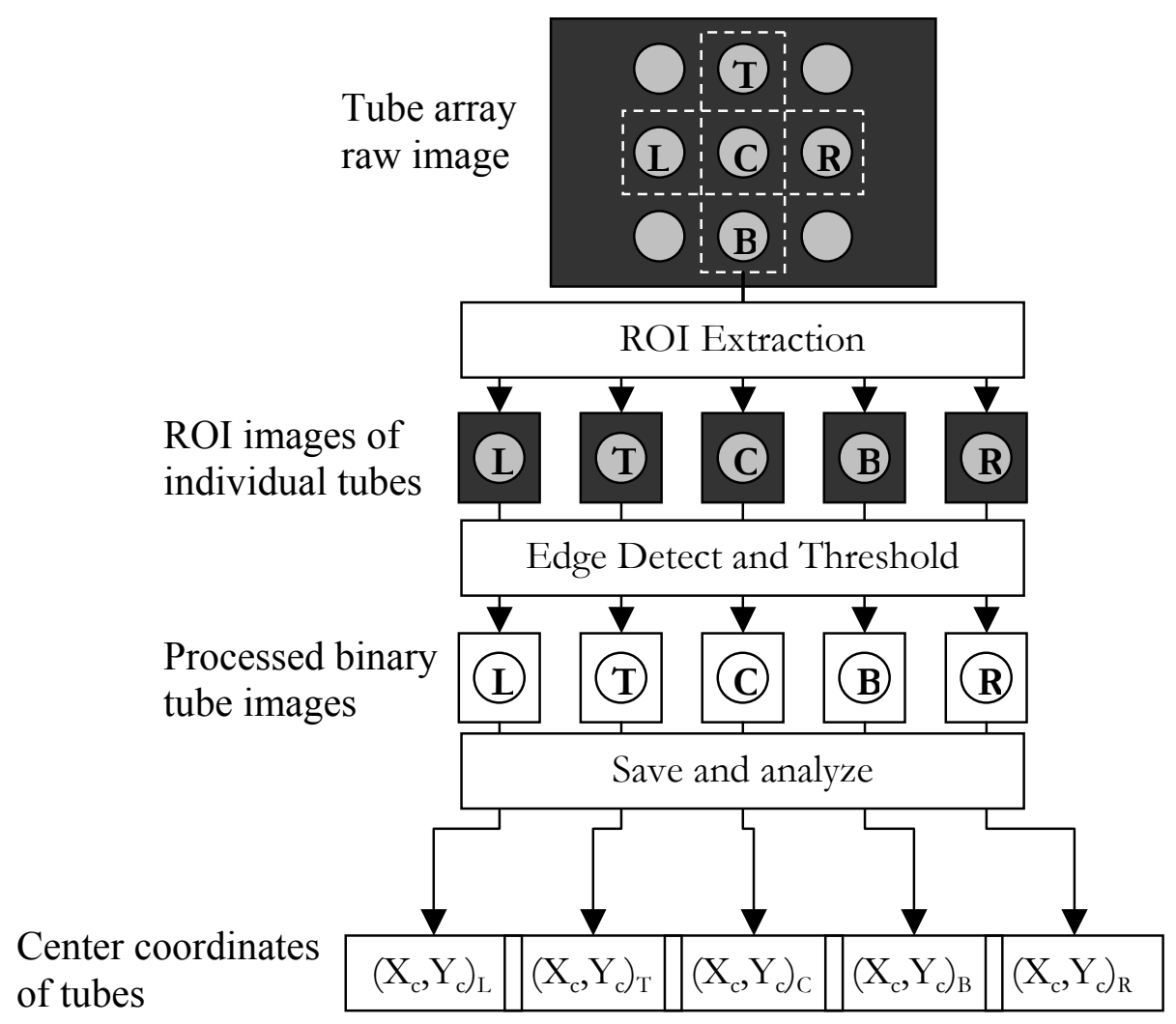

Figure 3.1 Schematic of the image processing procedure.

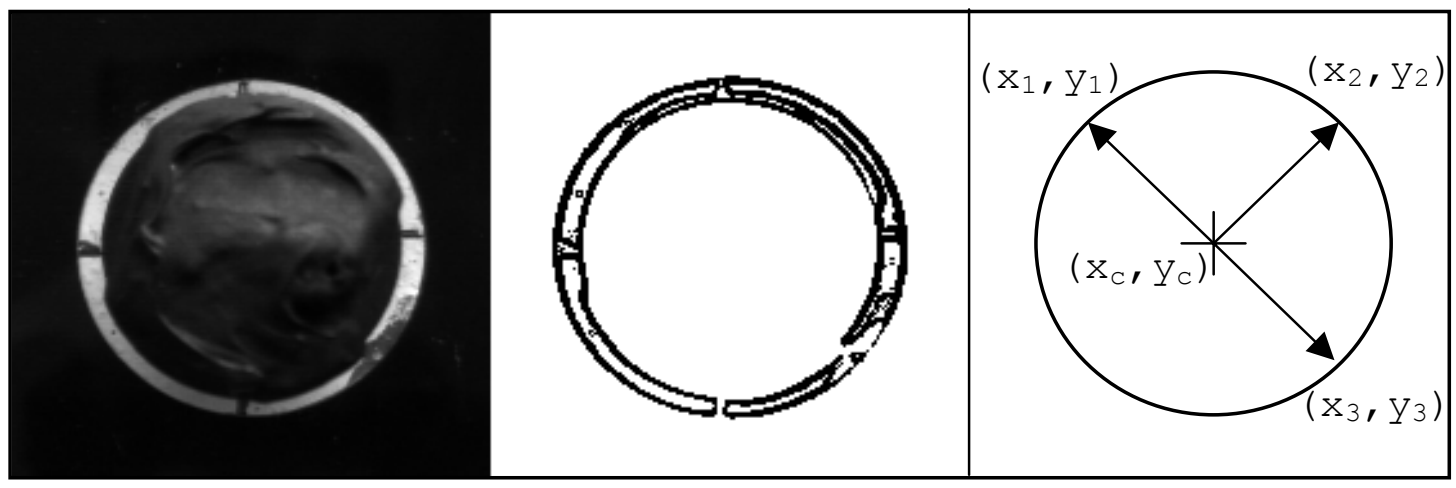

Figure 3.2 Left: the raw extracted ROI image of the tube profile; Center: binary tube image (after processing); Right: schematic of the circular interpolation using 3 perimeter points from the center image.

Sets of these small images can be differentiated by both the position of the tube within the array, and by the flow velocity during which the image series was recorded. The data processing routine is summarized in figure 3.1. The topmost part of the figure depicts a 3 by 3 subset of the vibrating tube array, with five of the tubes selected for processing and analysis (this is the case for both sets of the actual data). The tubes are labeled with letters according to their relative positions in the array: left, top, center, 
bottom, and right. Once a tube has been selected from the array (for a specific flow rate), the ROI images are automatically extracted, processed, and saved. The processed binary images are then automatically analyzed using either or both of the two analysis programs. Figure 3.2 illustrates the images at the various stages in the processing and analysis routine. The left image is an ROI image of the center tube, extracted from the raw array image. The center image is the result of the image processing and the left image is a representation of the tube location analysis using the 3-point interpolation method. This method and the cross-correlation analysis method are described in the following sections.

\subsubsection{Tube motion analysis: preliminary definitions}

Once the tube array images have been processed, the next important task is to locate and record the center position of each tube in the array, for each frame (i.e. for all times in the movie interval), relative to the equilibrium position. The equilibrium position of a tube refers to the center coordinates of the tube with no flow-induced displacement, i.e. the position of the statically suspended tube in still water. These equilibrium positions are determined using the images recorded at the (initial) zero flow rate. The Euclidean distance between the instantaneous tube center coordinates and the equilibrium position of a specific tube is referred to as the absolute displacement.

It was observed from early experiments that the displacement due to hydrodynamic drag of a tube subject to the water cross-flow is a significant component of the absolute displacement magnitude. Thus, for a fixed flow rate and, subsequently, a fixed reduced velocity, the tube would be displaced in the streamwise direction an amount proportional to the velocity. This displacement is approximately constant in time when averaged over a time interval significantly longer than the period obtained by dividing the characteristic length of the flow by the flow speed (in this case, the diameter of a tube divided by the maximum gap velocity). Using the total time of the movie as a basis, the time-averaged absolute displacement is calculated and referred to as the static or drag displacement. Subtraction of this static displacement from the absolute displacement gives us the definition for the dynamic displacement: the displacement of the tube about the average position of the tube during a time interval at a fixed velocity. The dynamic displacement is the component of motion that is identified with the actual vibration of the tube, that is, the time-varying, oscillatory component of the tube motion.

A diagram of the tube displacement definitions and parameters is provided in figure 3.3. The figure depicts the tube at various positions of interest. Point $A$ indicates the equilibrium position, a fixed reference point for all time (and all flow speeds). The circle-centers labeled with $t_{1}$ and $t_{2}$ indicate the instantaneous positions of the tube at two distinct times, and the circle labeled with $t$ represents a general position of the tube at some instant in the movie interval. The circle with center label $S$ is the static (drag) displaced tube position. Similar to the equilibrium position, the static displacement position is independent of time for a fixed flow rate, since it is the constant time-averaged component of the displacement. The vector $R_{S}$ measures the static displacement of the tube from the equilibrium position. The vector $R_{A}(t)$ is the time-varying absolute displacement, measured from the equilibrium center to the instantaneous center. The 
vector $R_{D}(t)$ is the dynamic displacement, measured from the static displacement position. Note that the static position $S$ is in fact the two-dimensional, spatial mean of the distribution $R_{D}(t)$ or, equivalently, $R_{A}(t)$.

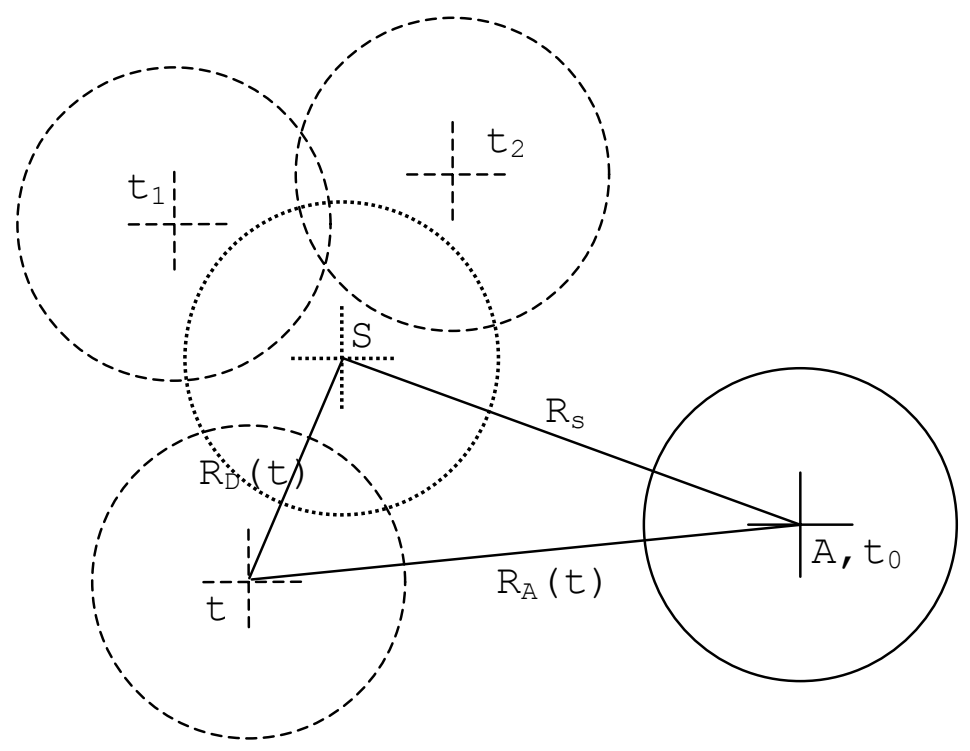

Figure 3.3 Diagram of the tube motion: static and dynamic displacement.

The magnitude of the tube vibration is the measure of interest within this experiment. Motivated by the previous definitions, the vibration of the tube may be closely associated with the dynamic displacement of the tube. The magnitude of vibration will be defined as the magnitude of the dynamic displacement vector, with the average vibration magnitude defined accordingly.

In order to locate the center of the vibrating tubes two different programs have been independently developed, based on two different methods, the "interpolation" and the "correlation" methods.

\subsubsection{Data Reduction: Interpolation Method}

The first program developed for the purpose of tube center location was done in the Java language and was built using ImageJ, a public domain image-processing library for Java provided by the NIH. The program takes a set of the processed, single tube images (typically about 1000 at a specified flow rate), uses a three-point interpolation scheme to locate the center coordinate of the tube in each image, calculates displacement, and saves the results as a text file. The actual computation of the center coordinates is simple. First, three points on the tube perimeter are located using an algorithm which checks for the outermost zero-valued pixels. Second, a circle is interpolated on the basis of the coordinates of the three points. This interpolation function returns the circle radius and center coordinates. The three points are chosen as far apart along the arc-length of the tube's outer edge as possible; this spacing is necessary for the accuracy of the 
interpolation. Finally, the center coordinates of the interpolated circle are taken as the center coordinates of the tube. The center coordinates of the tube at some specified time is equivalent to the instantaneous position of the tube, as referred to previously. A conceptual schematic of the interpolation is shown in figure 3.2 (above).

The interpolation returns the center coordinates of the tube within the frame of reference of the image. The origin of an image is typically located at the top left corner pixel of the image, with the positive y-axis pointing downward and the $\mathrm{x}$-axis identical to a normal right-handed system. Thus, each pixel of the image has corresponding positive $x-y$ coordinates indicating the pixel's position. The center coordinate of the tube is therefore calculated to be the pixel location closest to the mathematical center of the circular interpolation.

In order to calculate the absolute displacement of the tube at different times for non-zero flow velocities, the equilibrium location is determined as the center coordinates of the tube in still water (zero flow velocity). The absolute displacement is then equal to the Euclidean distance between the equilibrium coordinates and the instantaneous tube coordinates. This measure is independent of the frame of reference used to locate the tube, as long as it is consistent with the frame in which the equilibrium coordinates are based.

The list of center coordinates of the tube for the entire set of images allows one to compute the static displacement coordinate of the tube for the respective time interval. As stated, the static displacement coordinate is equal to the (two-dimensional) mean of the tube coordinate distribution. The dynamic displacement is then computed as the Euclidean distance between the static displacement coordinate and the instantaneous tube coordinate. Thus, the dynamic displacement can be measured only after the coordinates of the tube have been determined for all the images in the set. The program records the tube center coordinates, absolute displacement, and dynamic displacement for each tube image in a given set, and saves the resulting list to a text file. The text file is opened in Microsoft Excel, wherein the average values of the displacements are computed and the results of adjacent tubes are compiled together and compared.

\subsubsection{Data Reduction: Correlation Method}

A useful approach for the analysis of the tube displacement is image correlation. The correlation function is derived and closely related to the convolution integral. The convolution of two real functions, $f(\tilde{x})$ and $g(\tilde{x})$, is:

$$
f(\ell) \otimes g(x)=\int_{-\infty}^{\infty} f(\ell) \cdot g(f-\stackrel{\rho}{\rho}) d u
$$

and the cross-correlation is defined as:

$$
h(\ell)=f(\ell) \otimes g(\ell)=\int_{-\infty}^{\infty} f(u-\ell) \cdot g(u) d u
$$


A custom code for the implementation of a correlation function for matrices has been developed using Matlab. The main idea is to use cross-correlation to determine the displacement of the instantaneous tube image from the equilibrium tube image.

The advantages of using such a technique are twofold. First, the displacement is obtained without any directional ambiguity. Second, the cross-correlation output has a high signal-to-noise ratio, making the technique accurate and robust. On the other hand, for large images, the computation is extremely memory and time intensive.

In order to use the correlation function in Matlab, the data must be input in a numerical form, so the binary images are first converted into binary matrices. Black pixels $(0$ level) are given a value of 1 and the white pixels ( 255 level) are set to 0 ; the matrix indices correspond exactly to the pixel's image coordinates. This logical inversion of the pixel values happens to be more suitable to computation within Matlab. Furthermore, the image must be of equal dimension in both directions (square). There is no fundamental restriction on dimension for the correlation technique, however a square image is much simpler to handle.

The first step of the process is the auto-correlation of the equilibrium tube image, taken with zero flow, in order to set the reference point. The result of this operation is a matrix with dimension twice that of the original image. This is due to the fact that the correlation domain spans exactly twice the size of the input matrix in both $\mathrm{x}$ and $\mathrm{y}$ directions. The output is shown in figure 3.4: the peak represents the maximum correspondence of the image with itself; in other words the peak is located in the center of the matrix, where the image exactly overlaps itself.

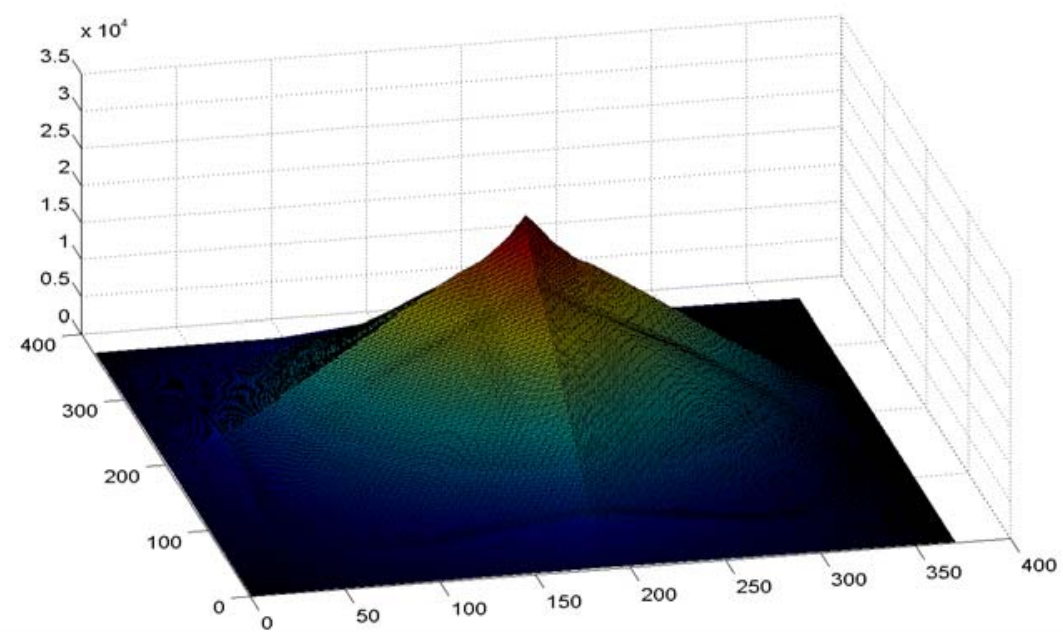

Figure 3.4 Matlab output. Auto-correlation of the reference image; the peak is located in the center of the output matrix. 
Each of the tube image matrices (again, typically 1000 per set) is then crosscorrelated with the reference matrix. The cross-correlation gives a similar output, but the peak is displaced with respect to the center of the array. This peak displacement represents the actual tube image displacement with respect to the reference image.

Subtracting the cross-correlation matrix from the auto-correlation matrix yields a matrix function shown in figure 3.5. The positive peak represents the center of the reference image while the negative peak represents the center of the displaced image. The difference between the coordinates of the two peaks is the absolute displacement of the center of the tube.

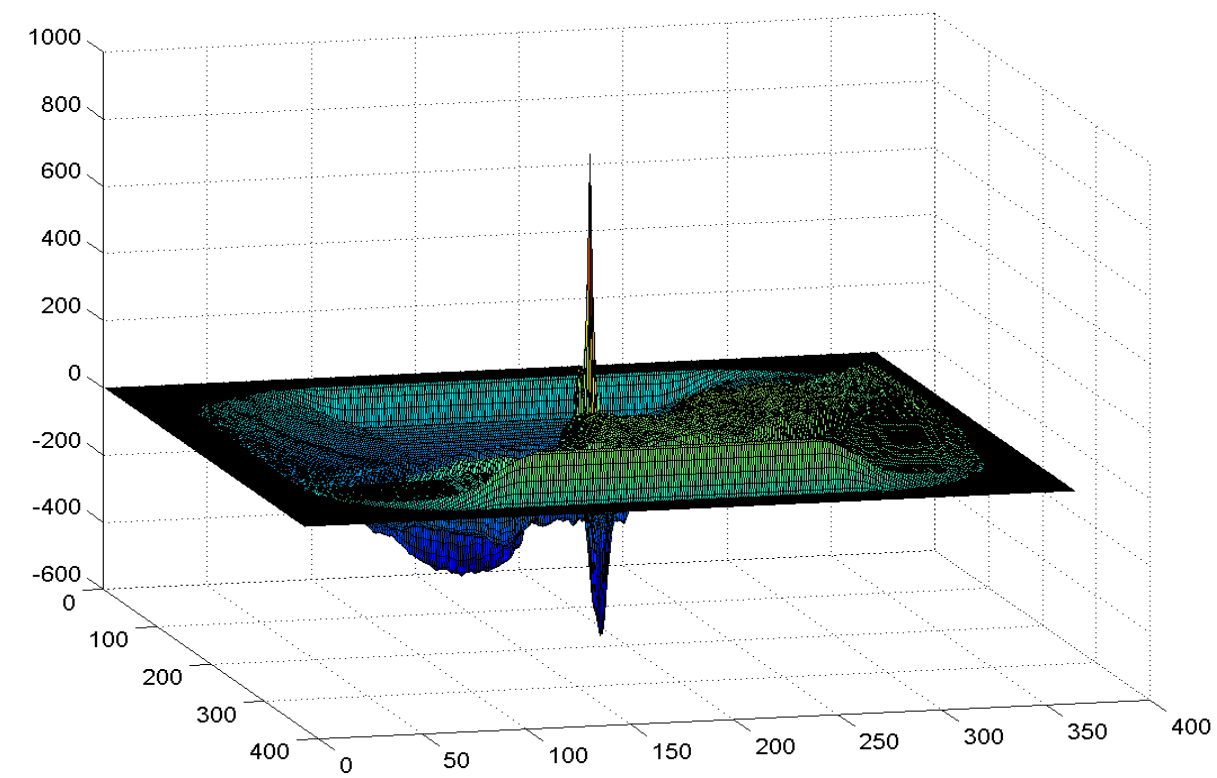

Figure 3.5 Matlab output. Cross-correlation matrix minus auto-correlation matrix.

Once the absolute displacements have been obtained in this way, the dynamic displacement may also be determined for each image using the same method as described in the interpolation technique. The same measures may be obtained through the application of either the correlation analysis technique or the interpolation analysis. Since the techniques are totally independent, the validity and accuracy of the two methods may be substantiated by examination of the results. 


\subsubsection{Results}

The main focus of the experimental work has been directed toward the study of the influence of two parameters on the onset of the fluid-elastic instability: the tube array configuration and the natural frequency of vibration of the tube array. Specifically, different sets of experiments were conducted on two different array configurations, normal square and normal triangular (figure 3.6). For each of these patterns, the natural frequency of vibration of the tubes was varied between three different values: 12,16 and $20 \mathrm{~Hz}$.
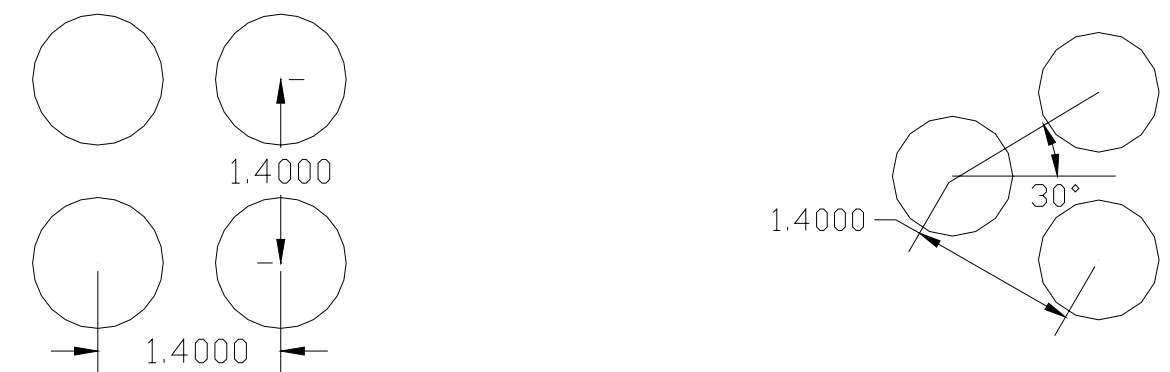

Figure 3.6 a) Normal square array configuration. Figure 3.6 b) Normal triangle array configuration.

After investigation of the normal square array configuration, a normal triangular pattern was chosen to provide a data set with which to compare. The pitch-to-diameter ratio of the triangular array is equal to that of the square array, allowing for a single parameter to be isolated and to investigate the effect on the FEI onset independently.

The first measure of importance is the identification and quantification of the onset of fluid-elastic instability. The two analysis methods (interpolation, correlation) were used for the analysis of the motion of seven adjacent tubes in the normal triangle configuration and five tubes in the normal square array. The tubes are named according to figure 3.7 , below.

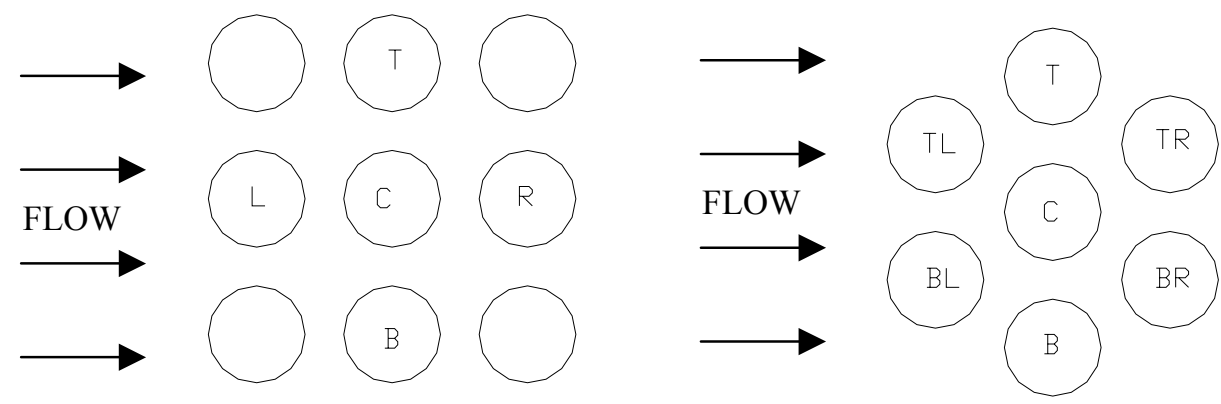

Figure 3.7 Normal square array (left) and normal triangular array (right). $T=T o p, C=C e n t e r$,

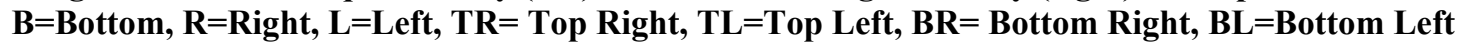


These tubes were selected (from the fifteen flexibly mounted tubes in the entire array) for observation because of their representative pattern and symmetrical distribution. Experimental characterization of this sub-group vibration may be comparable to a unit cell in a computational model. The behavior of the larger array of tubes may perhaps be determined by consideration of this sub-group as a basic component, where the geometric pattern of the larger array is formed by spatial extension (e.g. reflection or translation) of the sub-group pattern. Thus, it is of interest to consider such a "unit" group of tubes. The exact relationship between the behavior of this subgroup and a larger array of an identical configuration (i.e. a normal triangular array) is yet to be determined. However, it is hoped that observing the dynamics of the vibrations for this group will allow for a basic understanding of the flow-structure interaction and onset of FEI in this configuration.

For each reduced velocity, a time series of 600 images is recorded, corresponding to a time period of 5 seconds. The first data set (set 0 ) was taken for a reduced velocity equal to zero, in order to determine the static equilibrium position of the tube. For each configuration and calibration frequency a different number of points were taken. Both the interpolation and correlation methods were applied to each data set; since the two methods showed a good agreement, only results from the correlation method are reported.

\subsubsection{Normal Triangular Array}

The figures below show the behavior of the tubes vibration amplitude at different flow speed for the normal triangular array calibrated at 12, 16 and $20 \mathrm{~Hz}$.

Several features of the tube array vibration may be noted from the figures. In the first figure of the series a gradual increase and subsequent gradual decrease in the vibration amplitude may be observed within a reduced velocity range ( 0.75 to 2.5$)$ that can be related to the vortex shedding phenomenon. At this natural frequency, the energy associated with this particular mode of vibration is quite large compared to other cases, and the phenomenon is not as sudden as the one occurring when the array is calibrated at higher frequencies. The onset of instability appears to occur at a reduced velocity of about 2.5 for the majority of the tubes.

Figure 3.9 illustrates the behavior of the normal triangular array tuned to a natural frequency of $16 \mathrm{~Hz}$. Several distinct differences may be noted in relation to the same array at $12 \mathrm{~Hz}$. At a specific reduced velocity of about 1.4, a sharp increase in the average magnitude of vibration of the tubes occur; as noted before, this feature corresponds to vortex shedding. The magnitudes of these vibrations vary greatly from tube to tube in the array; it appears that the two tubes farthest downstream are the most excited, while the top and top left tubes are less than $20 \%$ of the average vibration magnitude of downstream tubes. The downstream tubes may be more excited due to the vortices originating from the upstream vibrating tubes. However, until the velocity field of the flow around the flexible tubes is known, the exact reason for this behavior may not be concluded. It is nonetheless useful to know that, in this array configuration, the downstream tubes are indeed more susceptible to vortex-induced vibration. The onset of 
instability occurs at a much higher reduced velocity (about 4 to 4.25 ) for this case and is a marked improvement over the $12 \mathrm{~Hz}$ tuning.

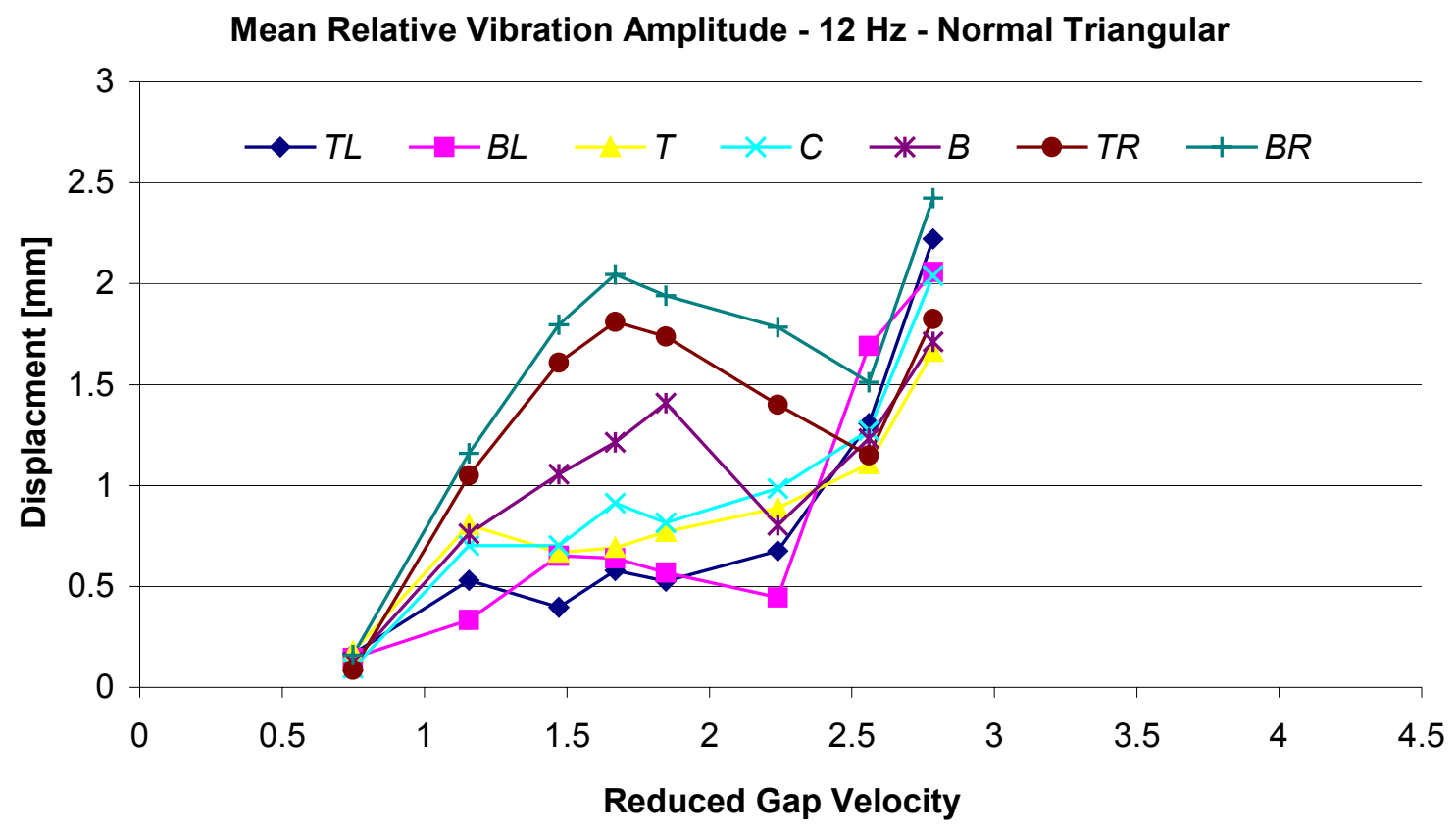

Figure 3.8

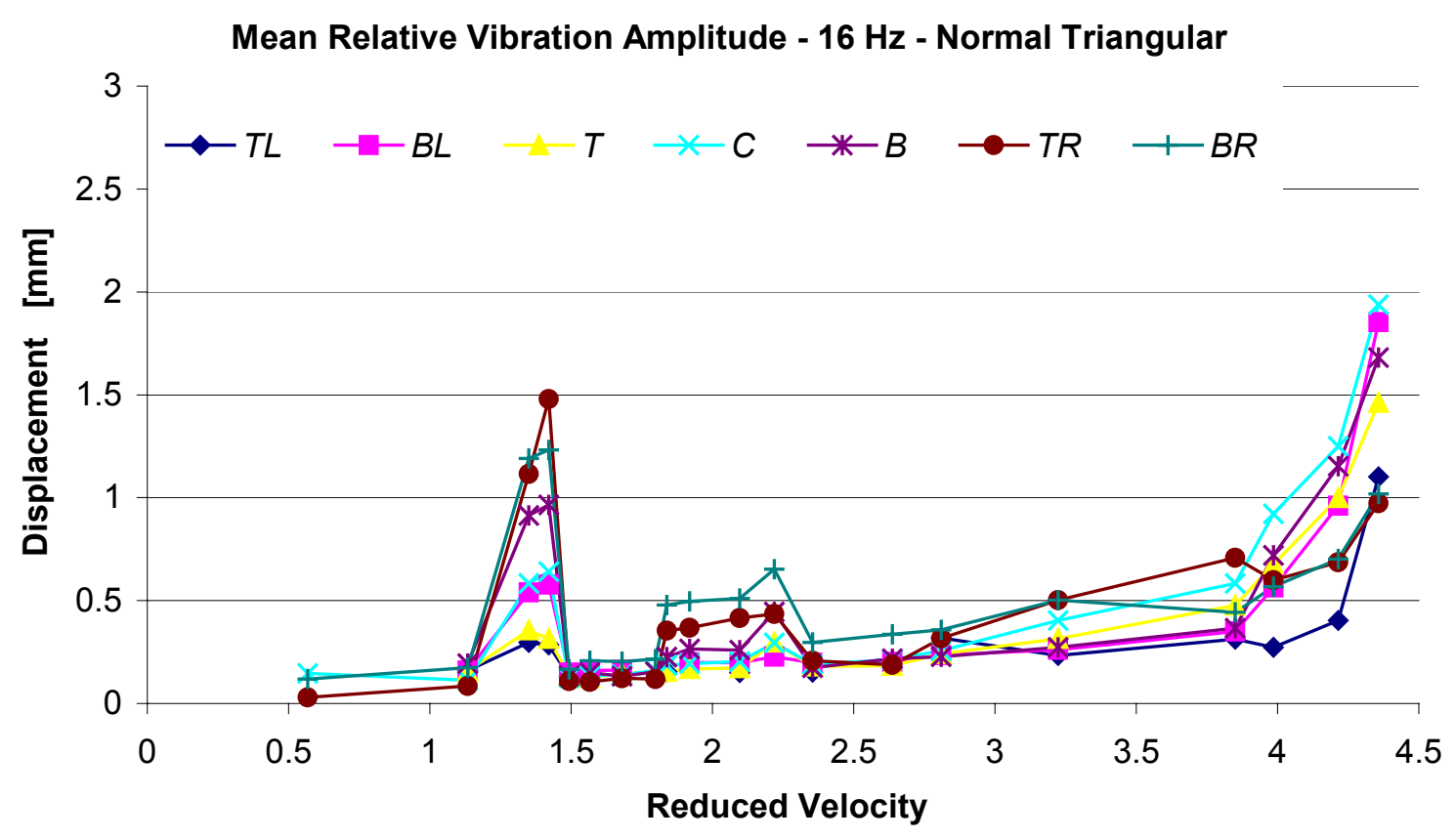

Figure 3.9 


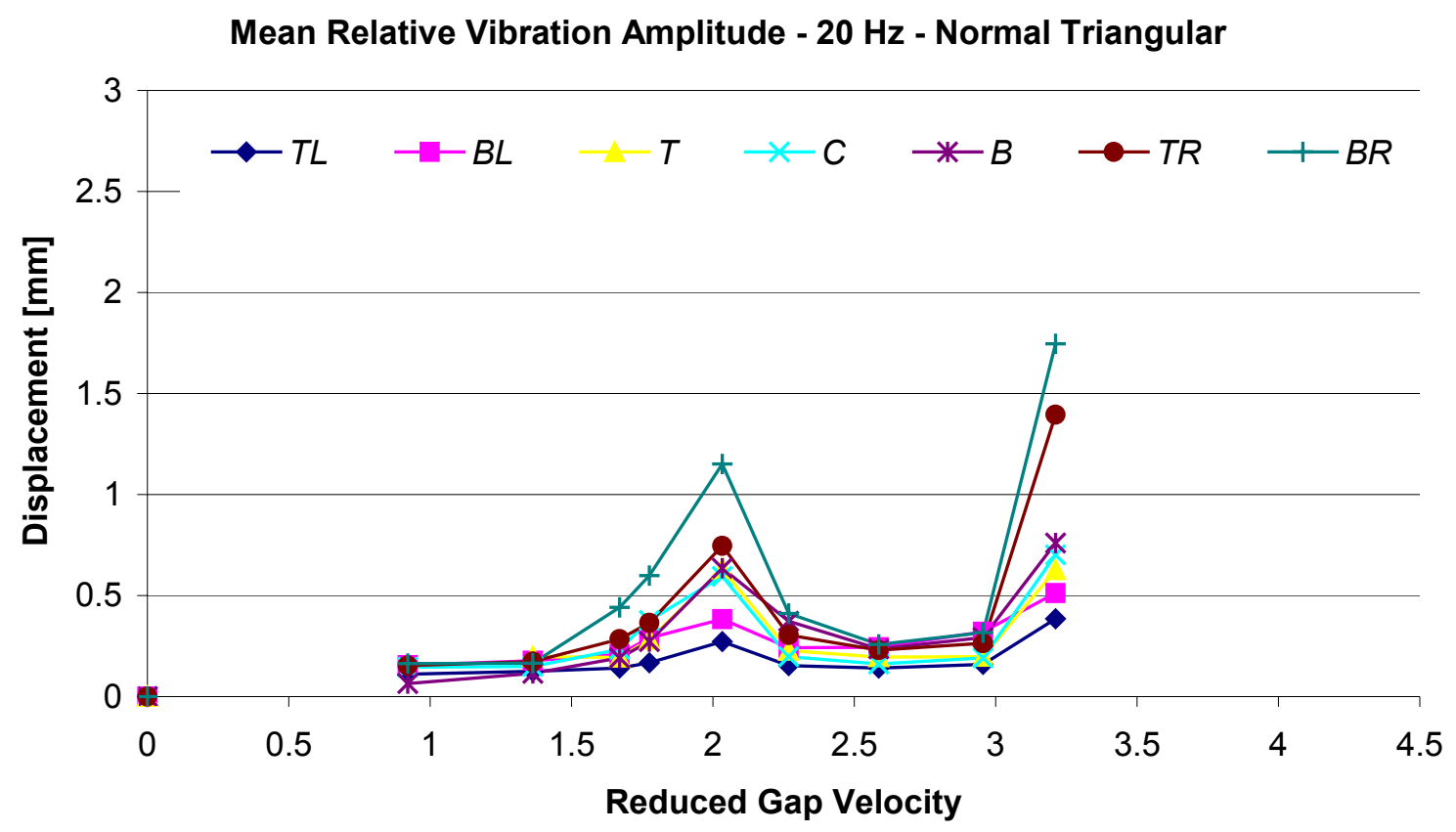

Figure 3.10

Finally, figure 3.10 illustrates the behavior of the normal triangular tube array tuned to a natural frequency of $20 \mathrm{~Hz}$. The magnitude of the vibrations is qualitatively similar to the previous two cases in the clear indication of the vortex shedding and onset of FEI phenomena. However, each tube in the array exhibits a more consistent range of vibration amplitudes, possibly due to the fact that the higher tension results in the tubes being very stiffly suspended and thus responding less to inter-structural forces and more directly to the fluid forces. The vortex-shedding peak occurs at the highest reduced velocity as compared to the other frequency cases, but the instability occurs at a significantly lower reduced velocity than the $16 \mathrm{~Hz}$ case. Thus it would appear that an optimal uniform tuning, i.e. one that suppresses the onset of instability until higher reduced velocities may be reached, exists based on the responses for this array configuration.

It may be further noted that the onset of instability manifests itself by the sharp increase observed in the vibration amplitude as a function of the inter-tube velocity; however, the onset differs substantially in this respect among the various array tunings. At the lowest natural frequency, the onset is observed almost uniformly across the array, with all the tubes increasing at a comparable rate. In the second case $(16 \mathrm{~Hz})$, although similar rates of vibration increase are apparent at the onset, the range of the magnitudes of vibration between the various tubes has noticeably begun to spread out. In the final case, the rate of growth of vibration amplitude at the instability varies widely across the array, roughly as a function of the streamwise location of the tube. Hence, at higher tunings of array natural frequency, i.e. design of tube arrays with greater stiffness, it appears necessary to focus attention on the influence of FEI on the downstream tubes and require a design that provides additional strength to these tubes. 


\subsubsection{Normal Square Array}

Some of the above observations can be made for the normal square array configuration. The experimental data for this arrangement are shown in the graphs below (figures 3.11 through 3.13).

Mean Relative Vibration Amplitude - $12 \mathrm{~Hz}$ - Normal Square

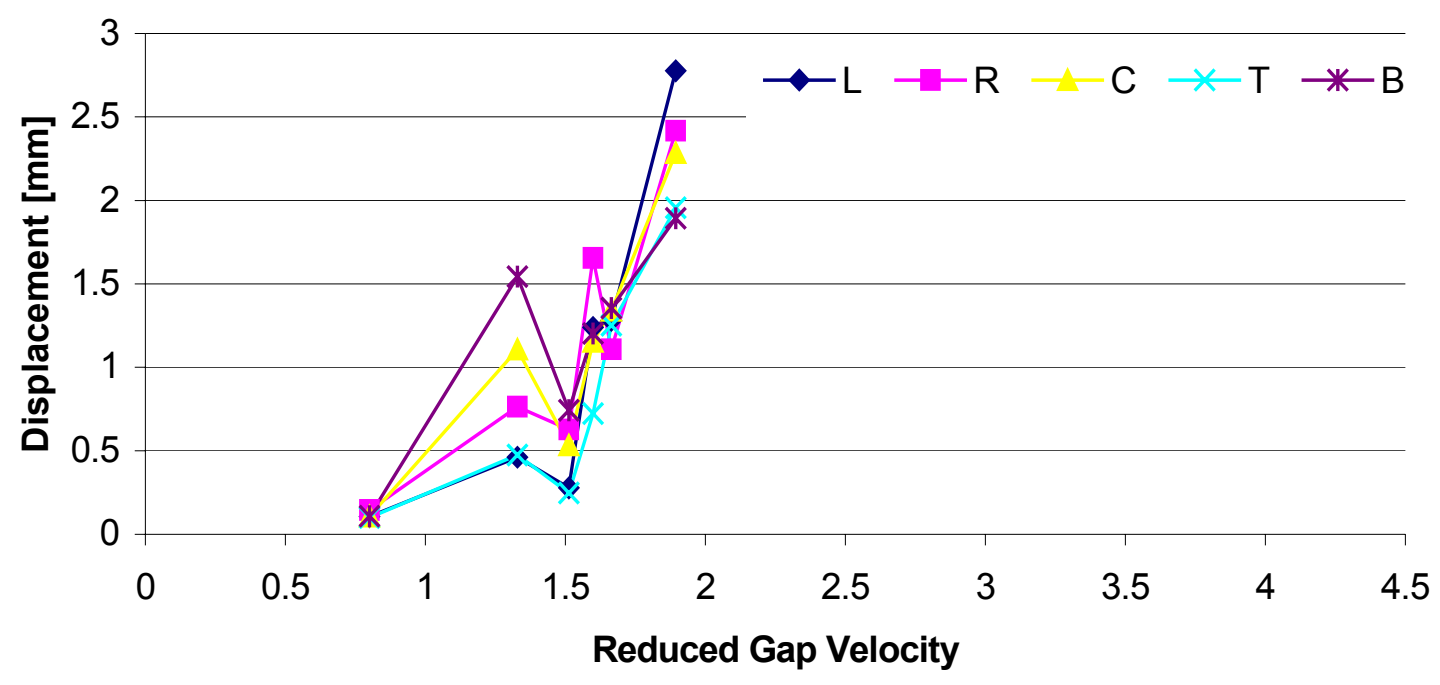

Figure 3.11

Mean Relative Vibration Amplitude - $16 \mathrm{~Hz}$ - Normal Square

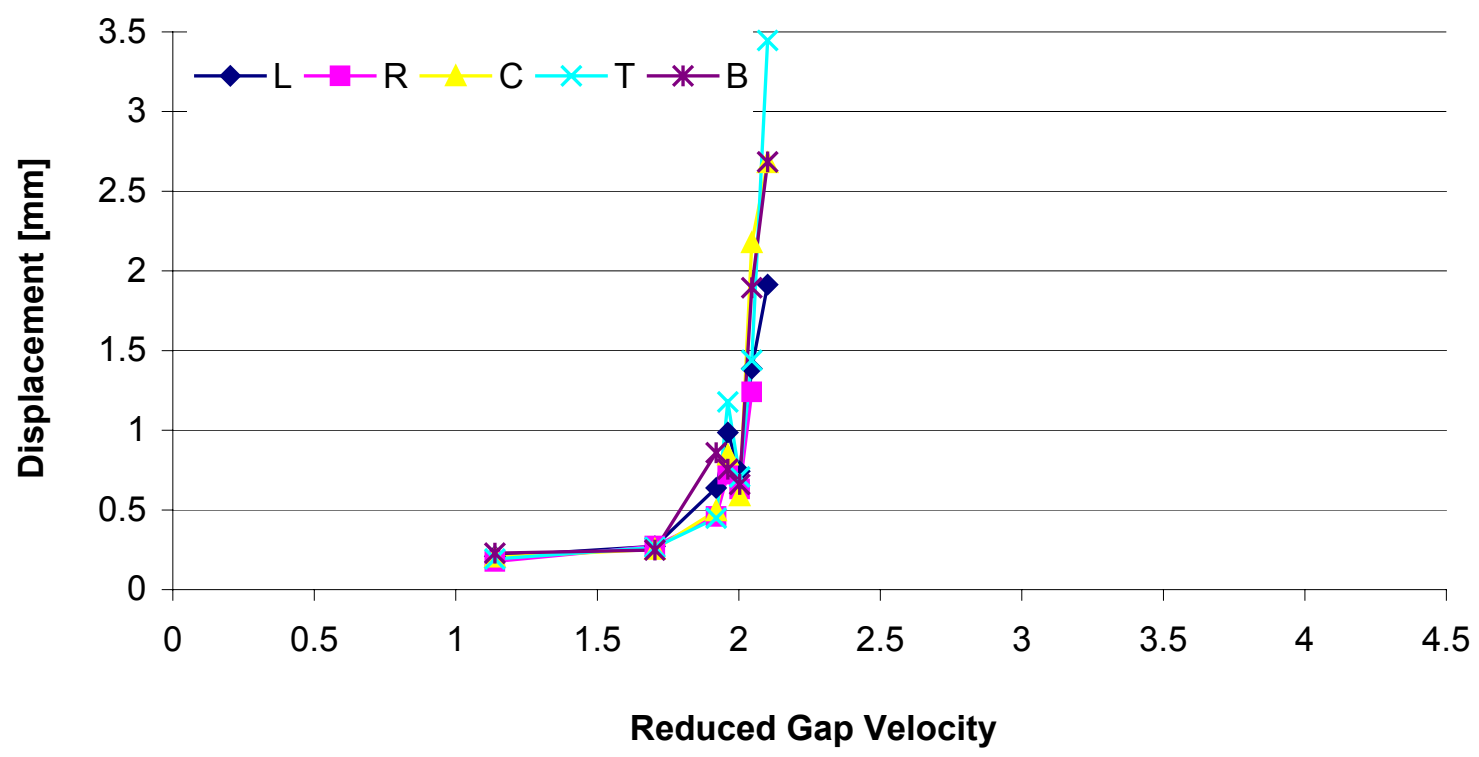

Figure 3.12 
Mean Relative Vibration Amplitude - $20 \mathrm{~Hz}$ - Normal Square

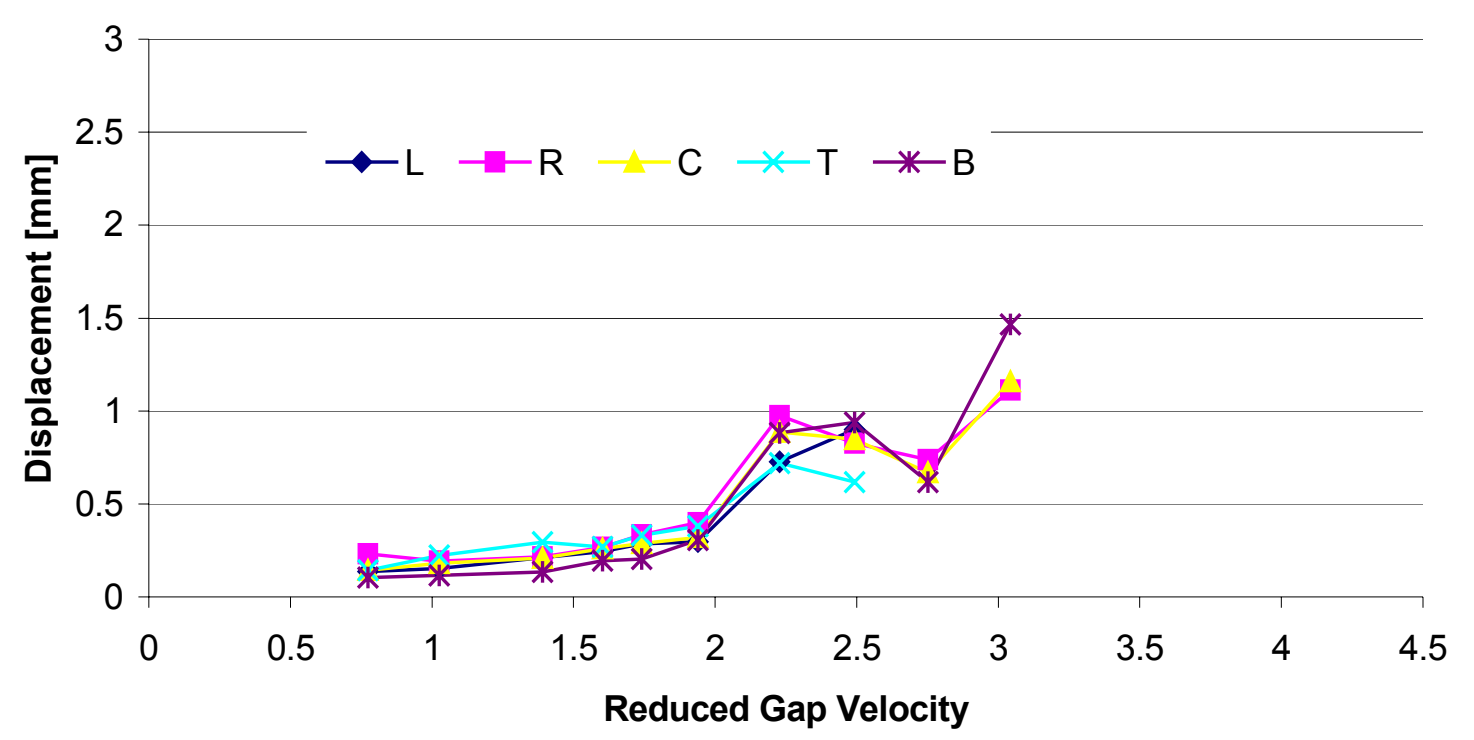

Figure 3.13

The first figure shows the vibration magnitude of the normal square array, with tubes tuned to a natural frequency of $12 \mathrm{~Hz}$, over several reduced velocities. The onset of FEI occurs at a relatively low reduced velocity of about 1.5 and seems to trigger all the tubes simultaneously. One interesting feature in this case is the presence of a spike or discontinuity in the growth of the vibration amplitude after the onset of FEI has begun. It was observed that the entire tube array vibration settled to a fixed amplitude and only increased significantly when the reduced velocity was forced much higher. The reason for this behavior is not clear, however the vibration of the tube array in this region was observed to be highly unstable already. The vortex shedding phenomena is observed at a reduced velocity of about 1.35; the magnitude of the vortex induced vibration is small in comparison to the magnitude of the FEI vibration for almost all of the normal square array configurations, in contrast to the normal triangular situation. Figure 3.12 depicts a similar onset behavior as the $12 \mathrm{~Hz}$ tuning, but more sudden and violent, with the vortex shedding peak almost indistinguishable from the dramatic onset of the FEI. It appears that the occurrence of the onset, though it actually occurs at a higher reduced velocity than the $12 \mathrm{~Hz}$ case, has a major influence of the vortex shedding: the instability seems to swallow the energy of the vortex mechanism, increasing the rate at which the FEI process takes over.

The final figure (3.13) stands in stark contrast to the previous experiments. At a tuning of $20 \mathrm{~Hz}$, the normal square tube array exhibits a much quieter response over the entire range of reduced velocities tested. Instead of a vortex-shedding peak, a "hump" in the displacement is observed. The vibration amplitude changes very gradually and the onset of FEI only appears to begin at a reduced velocity around 3, which is comparable to the normal triangular array responses. Further discussion and comparison of the two configurations is given in the following sections. 


\subsubsection{Vortex-Shedding and Frequency Response Observations}

The maximum displacements associated with the vortex shedding may appear large within the data presented, but are comparatively small in relation to the fluid-elastic instability normally encountered in similar experiments. Furthermore, the vortex-induced vibration mechanism is typically less persistent than FEI, tending to be more intermittent and diminishing quickly when the flow velocity is increased past the region in which the vortex shedding is prevalent. This region may be predicted through calculation of the Strouhal number for the tubes, and thus avoided by proper control of the flow speed. The Strouhal number, defined as

$$
S=\frac{f_{s} D}{U_{p}}
$$

may be calculated once the vortex shedding frequency, corresponding to the reduced velocity at which the resonance occurs, is measured. Since the exact, time-dependent displacement function is available, the structural vibration frequency of the tube may be directly calculated. Figure 3.14 shows the vertical displacement signal obtained for the center tube in the normal triangular array at the maximum reduced velocity. The maximum displacement is around $6 \mathrm{~mm}$, indicating that the phenomenon of fluid elastic instability is already established.

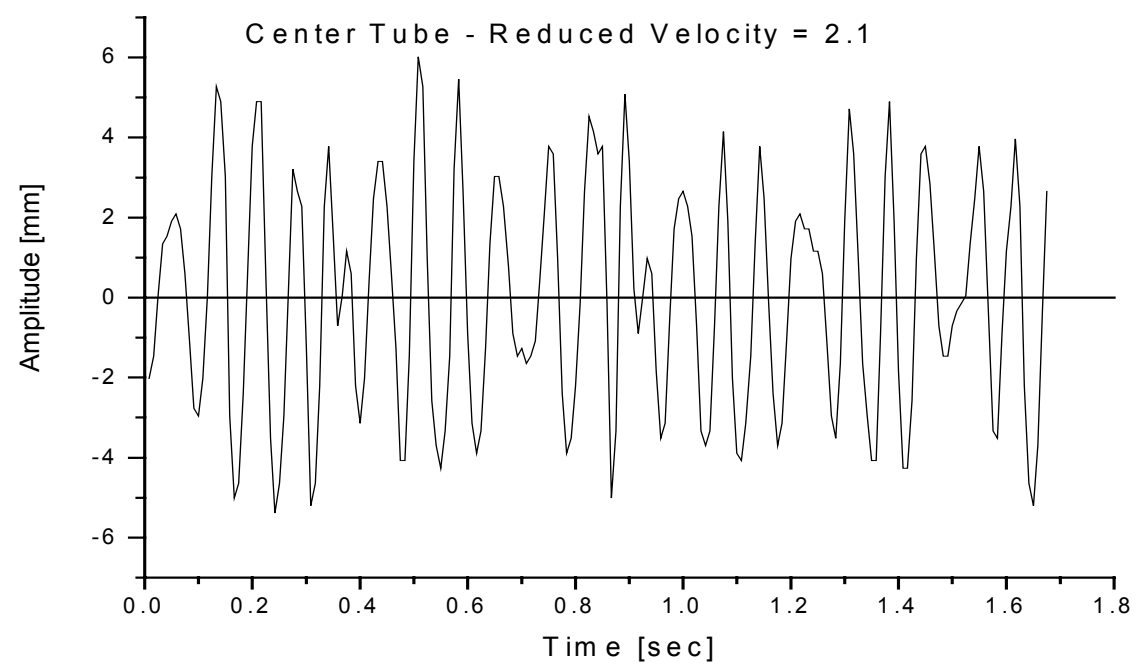

Figure 3.14 Amplitude of oscillation vs. time; vertical displacement of the center tube at Reduced Velocity $=\mathbf{4 . 3 5}$.

The Fourier transforms of these horizontal and vertical tube position functions were evaluated for the data at each reduced velocity, providing the tube structural frequency of vibration in both of the principal directions of motion. The vortex shedding frequency is then approximated by taking the average of these two structural vibration frequencies, and the Strouhal number is directly computed by the above equation. Using the information processed thus far, the Strouhal number was determined to vary between 0.4 and 0.5 . 

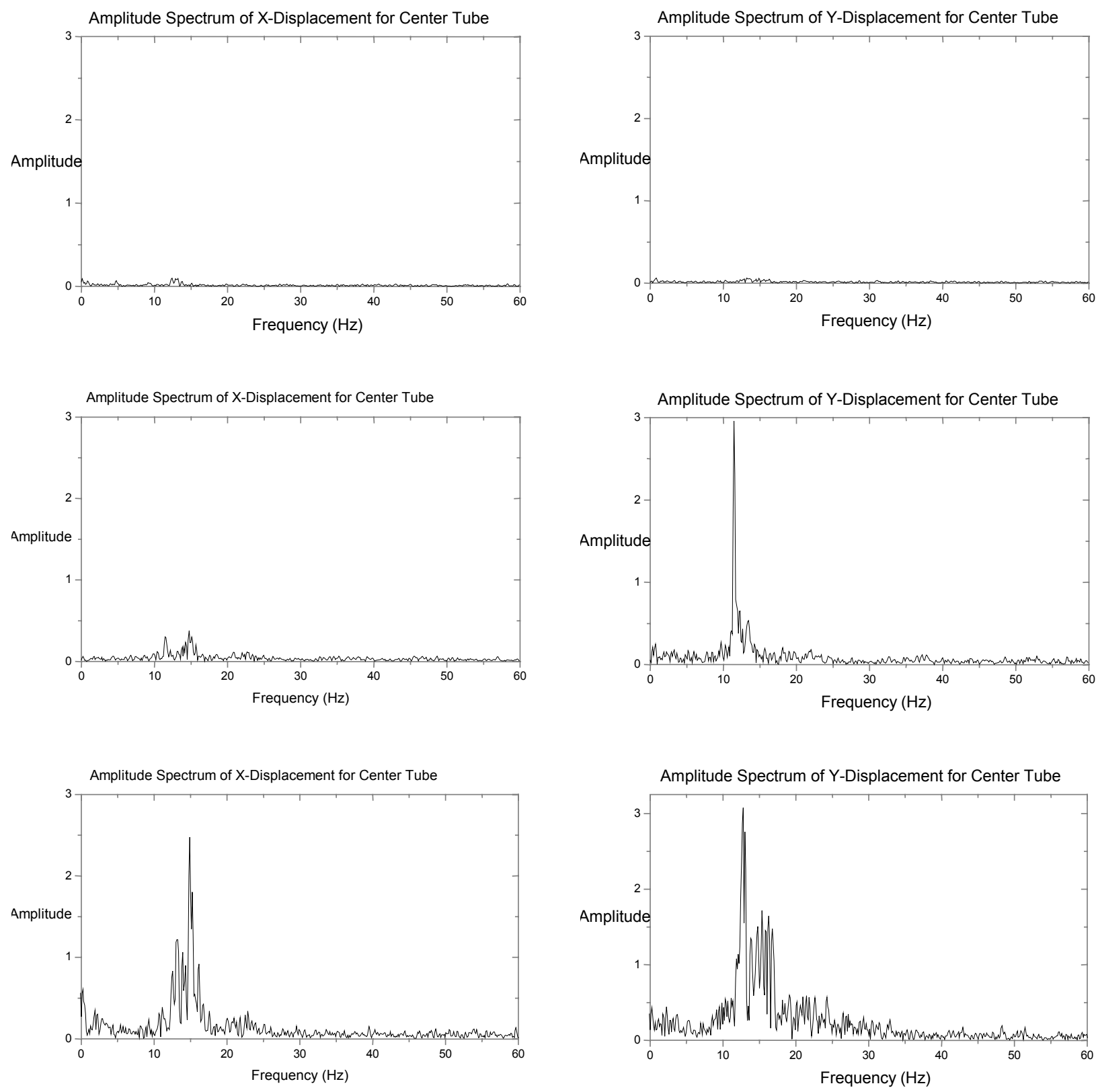

Figure 3.15 Amplitude Spectra for the center tube vibration in a normal square array (tuned to $16 \mathrm{~Hz}$ ), at reduced velocities of $0.57,1.96$, and 2.10 . The left-hand side plots correspond to the $x$ displacement frequencies, the right side corresponds to the $y$-displacement.

The amplitude spectrums for the center tube vibration, in both principal directions (vertical and horizontal), are shown in figure 3.15, for three important reduced velocities. This information is useful for not only identification of the natural frequency of the tube vibration in flow conditions, but also in characterization of the structural frequency response as the reduced velocity is changed. It is apparent that the energy spectrum of the tube vibration is centered near the tube's natural frequency; however, the distribution of the energy changes between the vortex shedding and the instability. 
The lowest reduced velocity shows negligible response across the entire spectrum. At a reduced velocity of 1.4, strong response around $12 \mathrm{~Hz}$ is observed in the $\mathrm{y}$ displacement function, but no significant response is observed in the x-displacement. This result is very indicative of the vortex shedding phenomena, wherein the major displacement is in the lift direction, and occurs at a vortex shedding frequency at or nearby the structural vibration frequency. The fact that the observed vibration frequency is lower than the natural frequency to which the tubes were calibrated is to be expected, since the tubes were calibrated to $16 \mathrm{~Hz}$ in air. The water flow environment apparently contributes a great deal to the total damping of the tubes, which is predicted by theoretical modeling.

Finally, the amplitude spectrum for the center tube undergoing FEI vibration is shown for a reduced velocity of 2.1. Note that both directions show strong responses, indicative of vibration in both directions. While both spectra exhibit well-defined peaks at notably different frequencies, the bandwidth of vibration is much more spread out than for the vortex shedding case. This would indicate the presence of several different excitation mechanisms contributing to the overall energy of the tube vibration. Future analysis must be done in order to determine exactly what components of the energy may be accounted for by the variety of possible excitation mechanisms (turbulence, coherent flow structures, inter-tube forces, etc.).

\subsubsection{Comparison between Array Configurations and Other Sources}

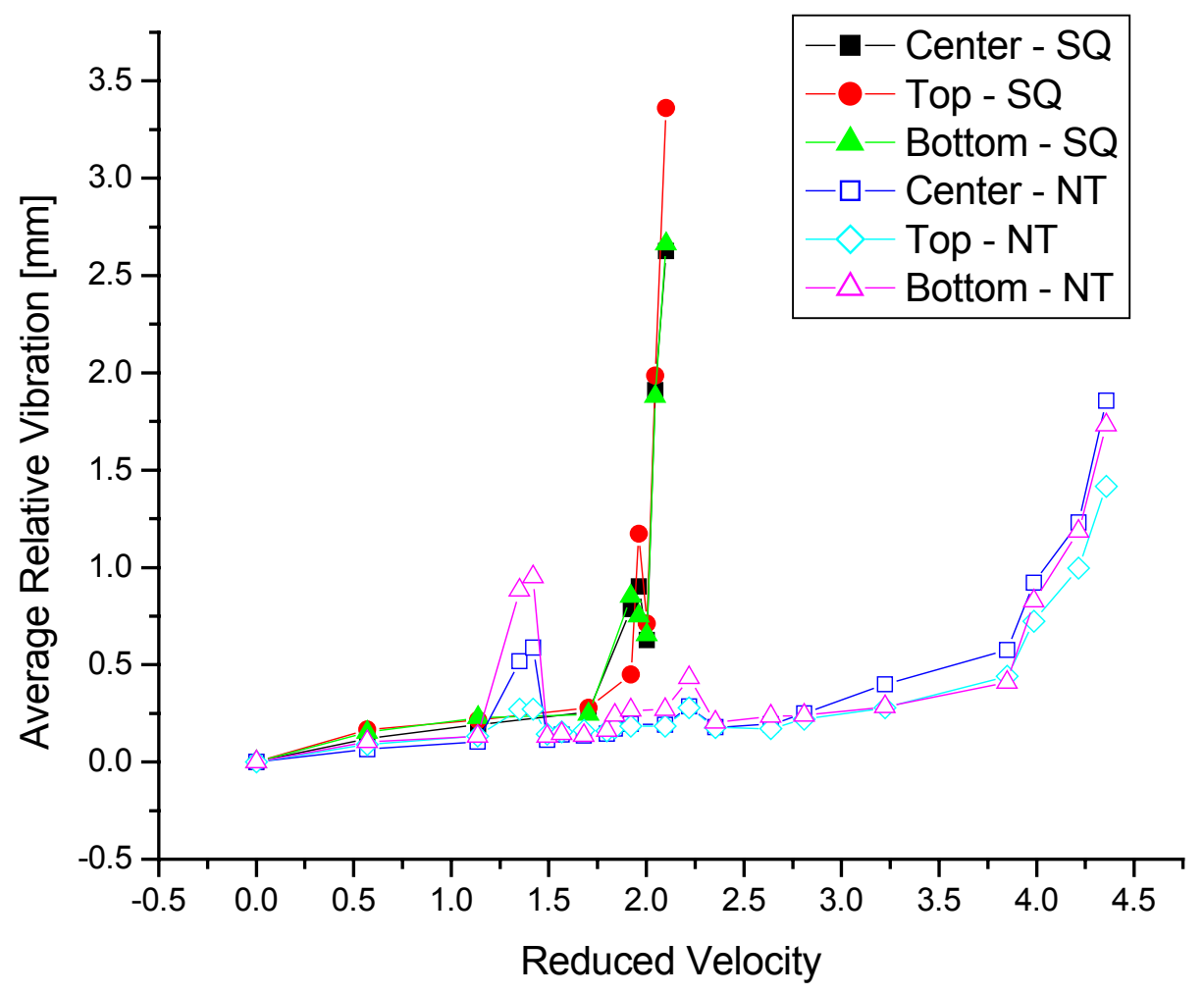

Figure 3.16. Comparison of FEI onset between the normal triangular array and the square array configurations. Data from the square array is labeled "SQ" in the graph legend. 
Several of the above observations of the normal triangular array vibration may be contrasted with the observations from the vibration of the square tube array. The average relative magnitudes of vibration for three tubes from both the square and the normal triangular array are plotted in figure 3.16.

In the data from these experiments, both the normal triangular and square tube arrays are of the same diameter $(25.4 \mathrm{~mm})$ tubes, pitch-to-diameter ratio (1.4), and natural frequency $(16 \mathrm{~Hz})$. The onset of FEI for the normal triangular configuration has been described above; by contrast, the onset of FEI for the square array occurs at a reduced velocity of about 2, whereas the onset for the normal triangular array occurs at twice the flow velocity. Furthermore, the instability of the square array occurs very violently and reaches maximum average vibration magnitudes $50 \%$ larger than those attained in the experiments using the triangular array; the instability in the triangular array occurs in a relatively gradual manner, with average vibration magnitudes of less than $2.0 \mathrm{~mm}$ at flow speeds of over twice those attained in the square array tests.

Another observation of interest is the location at which the vortex shedding occurs for each of the two configurations. The square array exhibits this phenomenon at a reduced velocity nearly coincident with its onset to FEI, whereas the vortex-shedding peak for the triangular array is separated from the onset of FEI by almost 3 units of reduced velocity. Since the reduced velocity depends only on the flow speed (in this case), this separation indicates that the two mechanisms take place under quite different flow regimes; the vortex shedding occurs at a Reynolds number of approximately 12886 , while the onset of FEI occurs at $\operatorname{Re} \sim 34923$. This difference may have some fundamental influence on the manner in which the instability evolves and help explain the differences observed in the FEI between the two configurations. Knowledge of the Reynolds number is not sufficient, however, to determine the nature of the fluid interaction in these two cases; a detailed measurement of the flow speed is again required to support any conclusion regarding the fluid mechanism responsible for the onset of FEI and its influence on the evolution of the instability.

Pathlines of vibration for the center tube from both the square and triangular configurations are shown in figures 3.17 and 3.18, respectively. The pathlines correspond to the motion of the tube over a short time period following the onset of instability. The distribution of the tube position coordinates also shows that the tube vibrates in a more or less random fashion; the tube position seems to be distributed evenly about the static displacement point for the data taken so far. This indicates that the forcing on the tube is isotropic under the test conditions. As for the graph of the square array, it can be seen that the tube experiences several sudden changes in direction, with particular preference to the vertical direction. 

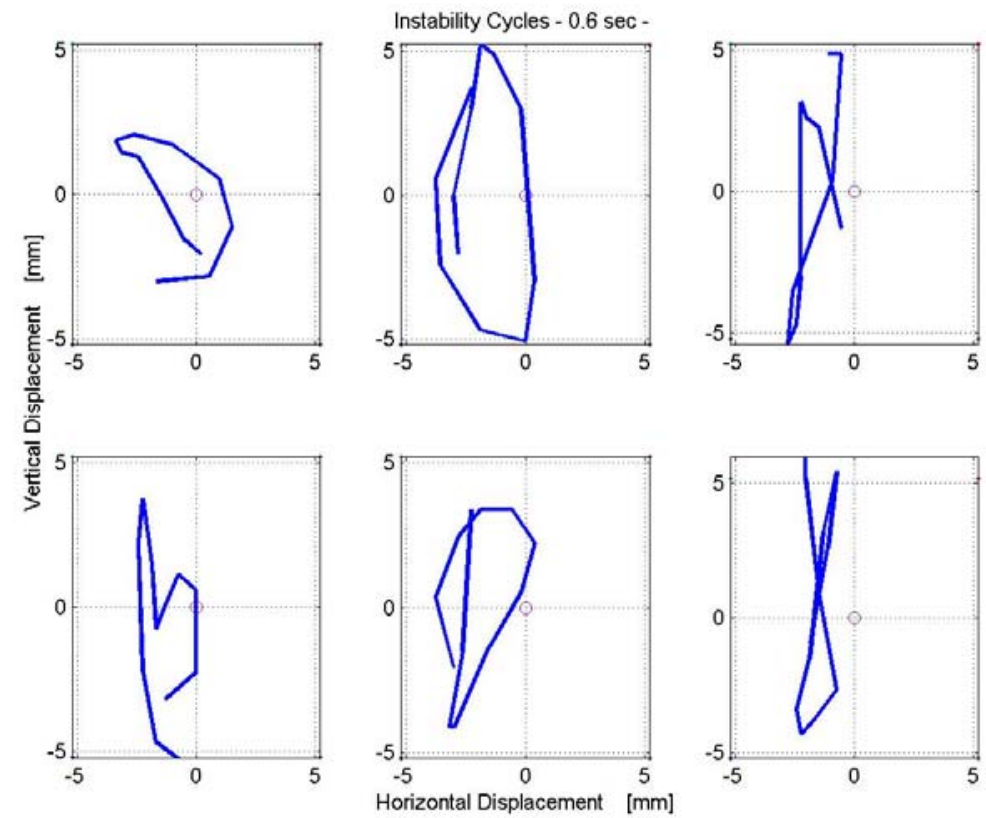

Figure 3.17. Pathlines of the center tube in a normal square array, coordinates over six sequential 0.1-second time intervals during the instability. The red circle indicates the equilibrium position. The flow is from right to left.
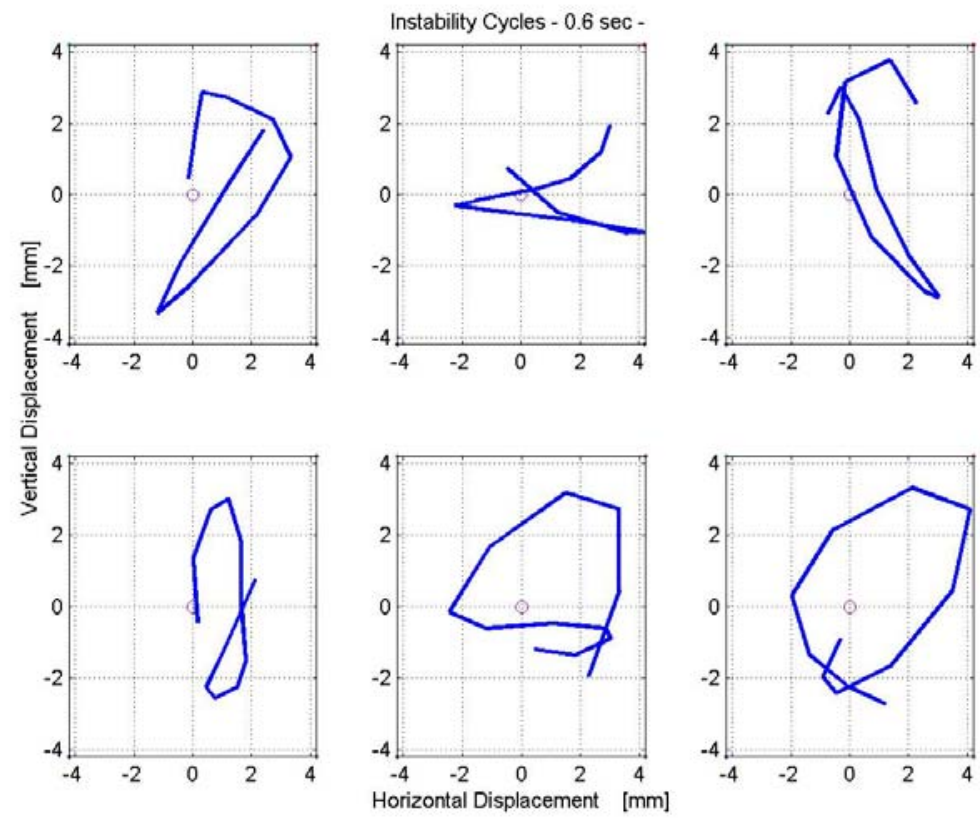

Figure 3.18. Pathlines of the center tube in a normal triangular array, coordinates over six sequential 0.1-second time intervals during the instability. The red circle indicates the equilibrium position. The flow is from right to left. 
The pathlines for the normal triangular array depict a motion more accentuated in the drag direction and an orbit more clearly circular. This behavior in the center tube of the triangular array can be attributed to a more significant "confinement effect" due to the geometry of the array. In the triangular configuration, the center tube is surrounded by six tubes located at the same distance, while in the square array there are only four. Furthermore, in the square arrangement the fluid flow does not directly excite the tube vibration in the vertical direction, but induces this motion via an intra-tube flow mechanism: the flow displaces the tube, thus separating two tubes in the vertical direction, the velocity decreases because of the increased gap between the tubes and the restoring pressure increases. This phenomenon grows in an unbounded manner, causing the vibration to develop mostly in the vertical direction and resulting in violent clashing between adjacent tunes.

The experimental results were compared with available literature results regarding the same type of experiment. In particular, it was possible to construct an instability map where the onset of fluid elastic instability, regarded as the flow critical reduced velocity, is plotted against the mass damping parameter as defined previously. Figure 3.19 reports data from other authors (Weaver et al., 2000 ) for experiment in single phase flow. The graph also illustrates the suggested guidelines for tube bundle design and operation recommended by the ASME Boiler Code (Section III, Appendix N) and the median curve that best interpolates the experimental data. The points corresponding to the triangular array lie entirely above the mean curve, thus indicating that two different criteria should be used for the two different geometries of tube array configurations investigated in this study. In fact the recommended curve appears to be too conservative when applied to the normal triangular configuration; the consequence of an over conservative design results in a lower efficiency of the component, therefore causing economic losses.

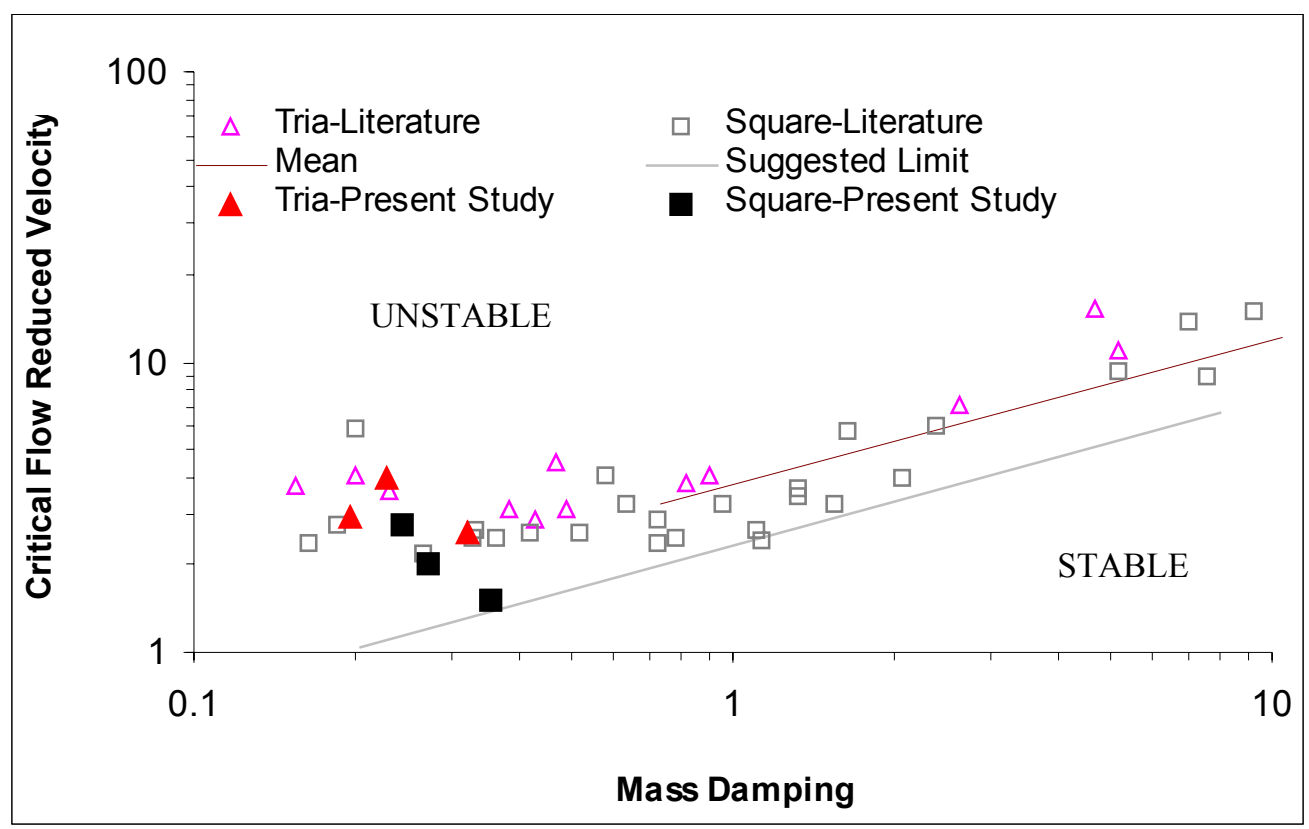

Figure 3.19 Instability map for single phase flow experiments, comparisons. 


\subsection{Two-Phase Experiment}

\subsubsection{Preliminary Results}

Prior to carrying out the actual fluid-elastic instability tests, a number of experiments to validate the experimental methods described in the previous chapter were carried out. Preliminary results from such experiments are discussed in this chapter. These results include the measurement of frequency and damping ratio, tube displacement, and freestream void fraction.

\subsubsection{Frequency}

The frequency of tube vibration was measured using strain gauges using the methodology described in the previous chapter. In order to determine the accuracy and precision of the strain gages in measuring the frequency, a simple test using a mass loaded cantilever beam was performed as shown in Figure 3.20 below:

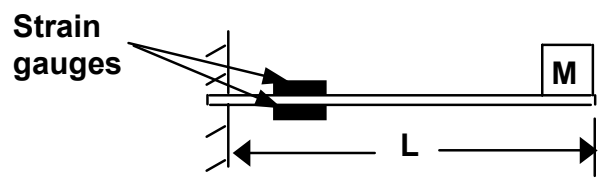

Figure 3.20: Mass loaded cantilever beam setup

The cantilever beams were made of stainless steel. They were $74.5 \mathrm{~mm}$ long, $7.4 \mathrm{~mm}$ wide, and $0.508 \mathrm{~mm}$ thick. The cantilever beams were clamped on one end and a mass (precisely cut lengths of 0.5 inch diameter brass rods) was attached to the other end. Strain gages were bonded to the top and bottom side of the cantilever beam, $6 \mathrm{~mm}$ from the clamped end and connected in a half bridge configuration. The mass that was attached to the end of the cantilever was gently tapped to set the cantilever in motion. For such a configuration, the natural frequency can be analytically calculated as

$$
f n=\frac{1}{2 \pi} \sqrt{\frac{3 E I}{M L^{3}}}
$$

where $f_{n}$ is the natural frequency of the system, $E$ is the modulus of elasticity of the beam material, $I$ is the moment of inertia, $L$ is the length of the beam, and $M$ is the mass attached to the end of the cantilever. Tests were carried out for 6 different masses. The results are summarized in Figure 3.21. 


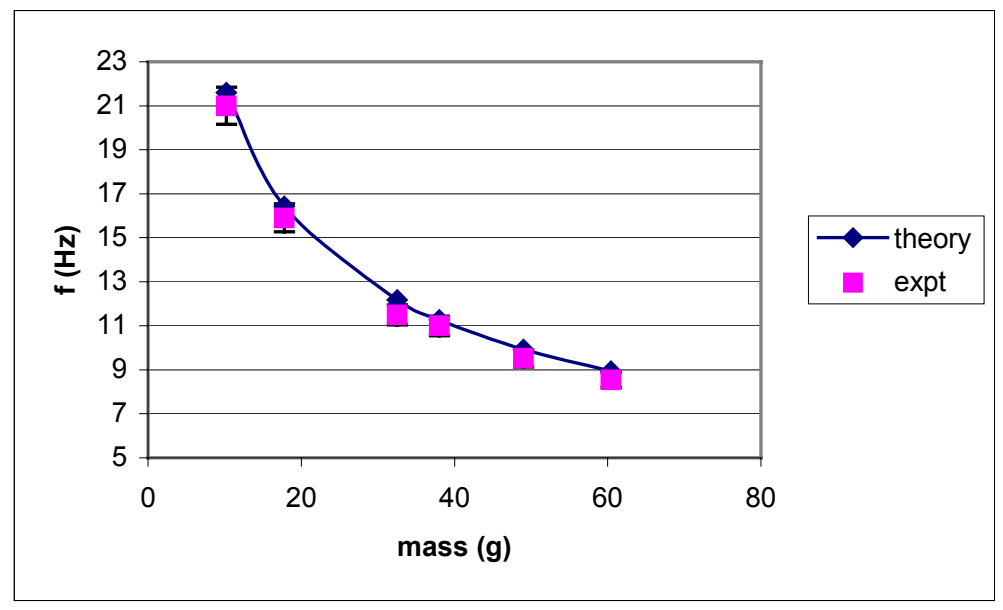

Figure 3.21: Natural frequency of cantilever-mass system

The uncertainty in the theoretical calculation is estimated to be $\pm 4 \%$. It can be seen that the experimental frequencies determined using the strain gauges match those predicted by theory. The experimental frequencies were determined by observing the amplitude spectrum of the strain gauge signal. The frequency of the tube-wire system is determined in a similar way. The natural frequency of the test tubes was determined outside the test section by constructing an aluminum frame that housed the test tube bundle. Figure 3.22 shows a photograph of the test bed used for the tube calibration.

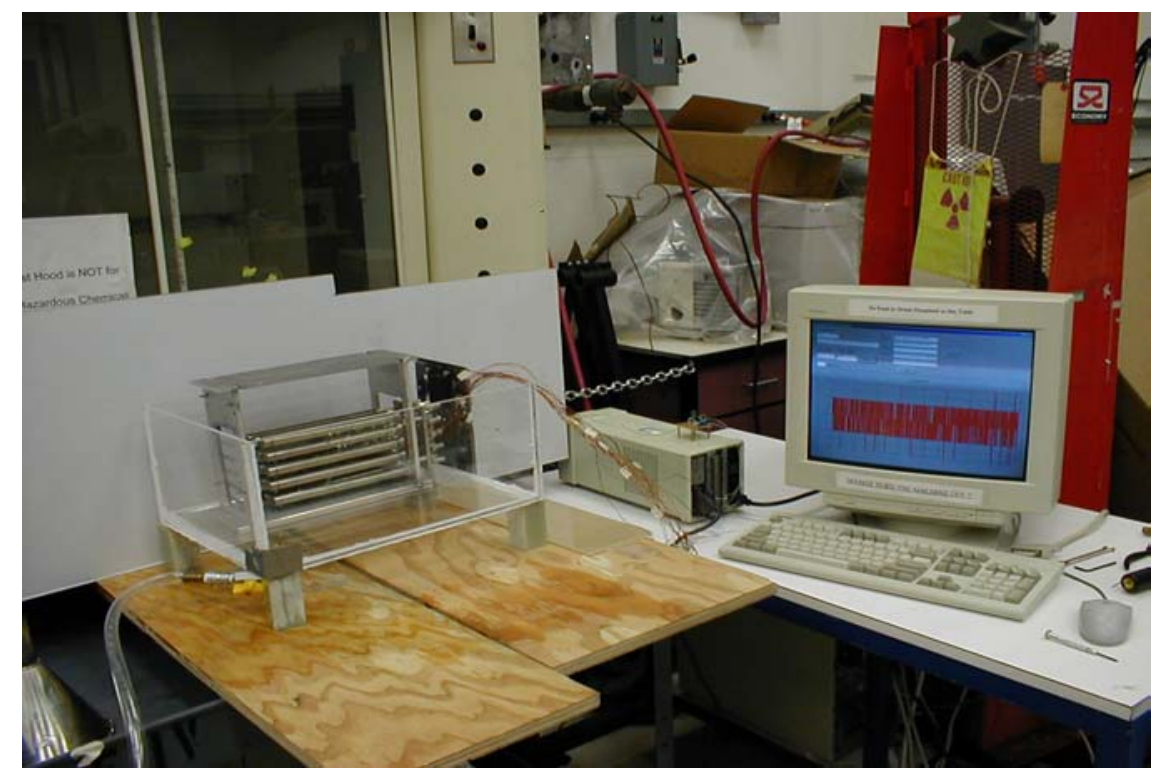

Figure 3.22: Test tube calibration set-up

The tube was gently tapped and the decaying vibration signal was recorded. As before, the amplitude spectrum of the strain gauge signal yielded the frequency of tube vibration. The test tubes were found to vibrate with a frequency of between $20-22 \mathrm{~Hz}$ in air and between $16-20 \mathrm{~Hz}$ in still water. A sample strain gauge signal output and its corresponding amplitude spectrum obtained for a tube-wire system are shown in Figure 3.23 . 

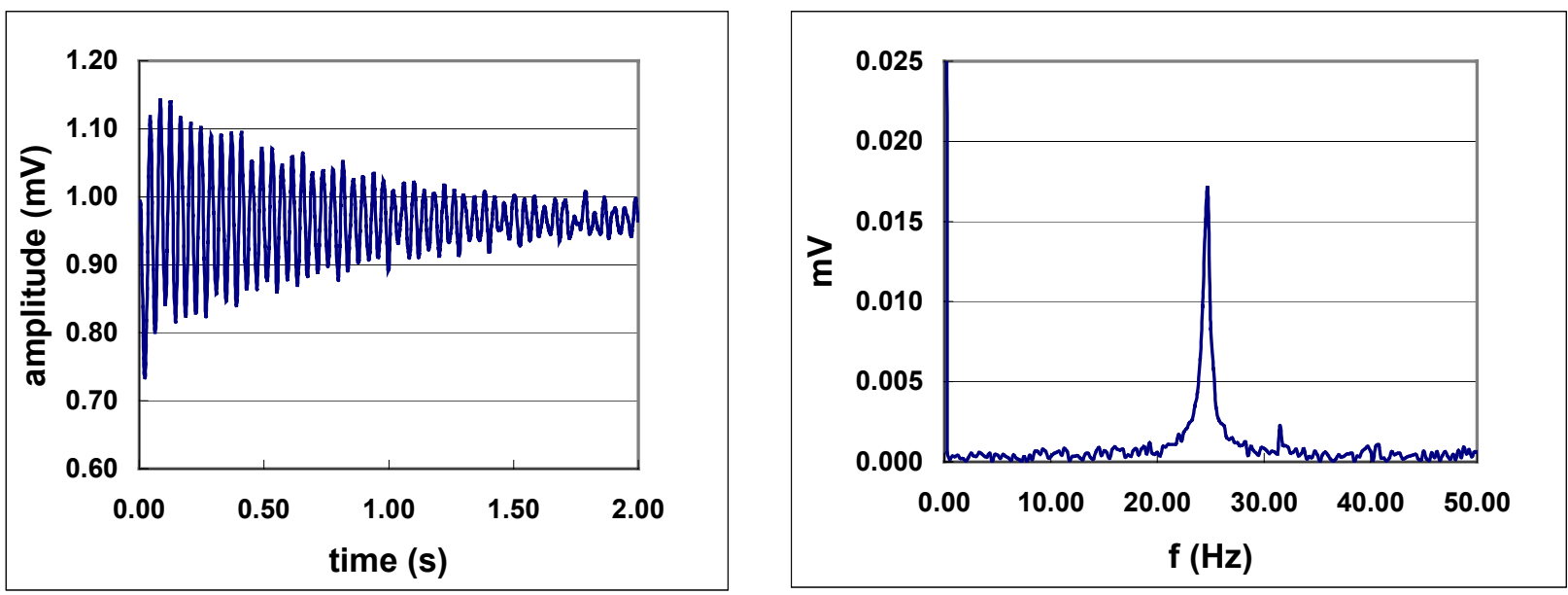

Figure 3.23: (a) Strain-gage signal of tube-wire system; (b) Amplitude spectrum

\subsubsection{Damping ratio}

As a first point of comparison, the damping ratio of a cantilever-beam-tube system as used by Joo and Dhir $(1994,1995)$ was measured. For this purpose, a hollow acrylic tube $27 \mathrm{~cm}$ in length and weighing $0.061 \mathrm{~kg}$ was suspended from its ends using stainless steel cantilever beams. The dimensions of the cantilever beams $(53.5 \mathrm{~mm} \times 7$ $\mathrm{mm} \times 0.5 \mathrm{~mm}$ ) were chosen to match those of Joo (1994). Strain gages bonded on opposite sides of the beam near the clamped end measured the vibration. Once again, the tube was gently tapped and the strain gage signal corresponding to the decaying tube vibration was recorded. The damping ratios were calculated using the logarithmic decrement method. The uncertainty of these measurements using this method was found to be $\pm 6 \%$. The results are shown in Table 3.1 and show good agreement with those obtained by Joo and Dhir, especially in air.

Table 3.1: Comparison of damping ratios obtained by Joo and present study

\begin{tabular}{|l|l|l|}
\hline \multicolumn{1}{|c|}{ Damping ratio $(\zeta)$} & \multicolumn{1}{|c|}{ Joo } & $\begin{array}{c}\text { Study with cantilever- } \\
\text { tube system }\end{array}$ \\
\hline Air & $0.008 \pm 0.001$ & $0.008 \pm 0.0005$ \\
\hline Water & $0.027 \pm 0.005$ & $0.022 \pm 0.001$ \\
\hline
\end{tabular}

The dummy test frame used for frequency calibration of the tubes was used to obtain some preliminary data on the damping ratio, , of the tubes. The tests were carried out for a single test tube, and a test tube located in the center of an array of 9 tubes. The central test tube was instrumented with strain gages to measure the damping ratio. The tests were carried out in air as well as in water. The damping ratios were calculated from the time history signal of the strain gages using the modified logarithmic decrement method as used for the cantilever-tube system. Once again, the test tube was gently tapped and the strain gage signal recorded. The results from these tests are shown in Figure 3.24. 


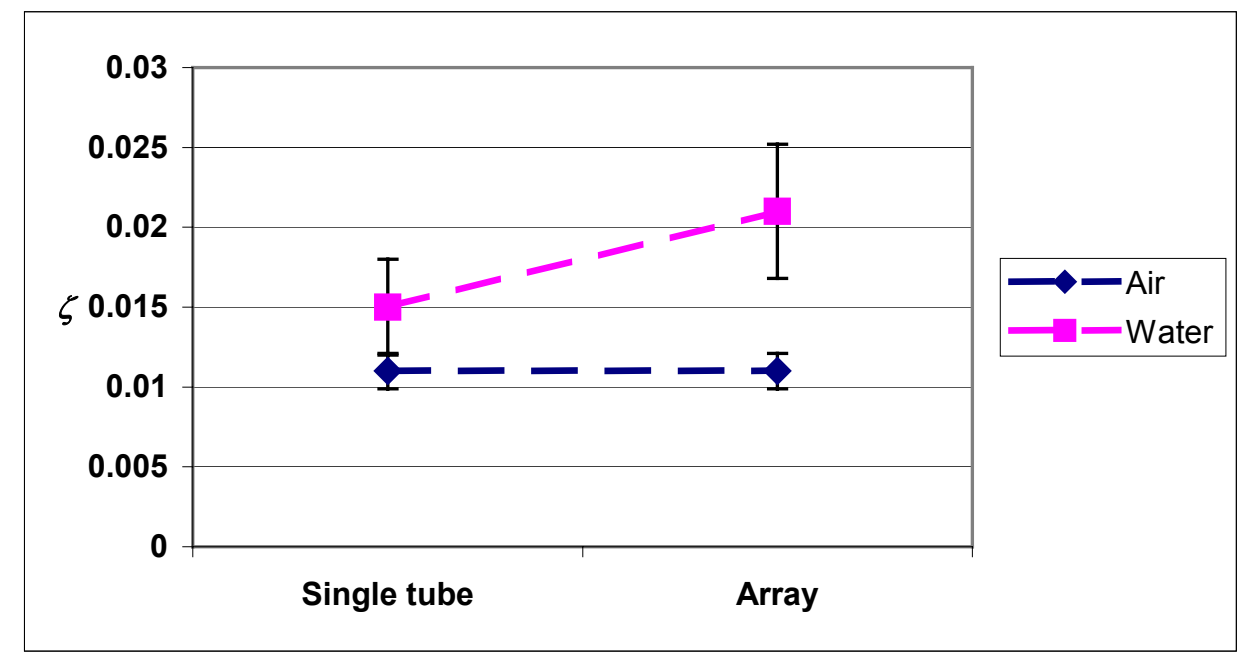

Figure 3.24: Repeat damping ratio tests with tube-wire system

The above results show that the damping ratio of the tubes in water is much higher than in air. This is expected and is attributed to the higher density of the fluid surrounding the tube. Also, in going from a single tube to an array, the damping ratio increases. This is particularly noticed in the case of water. Although the uncertainty of the measurements does not allow a definite conclusion, this could be due to the "confinement effect" caused due to the movement of the incompressible fluid and the neighboring tubes surrounding the test tube. The confinement effect is a function of the geometric arrangement of the tubes and can be expressed as a factor multiplying the damping ratio for a single tube. The multiplier is expressed as (Blevins, pp. 309)

$$
\frac{1+\left(D / D_{e}\right)^{3}}{\left[1-\left(D / D_{e}\right)^{3}\right]^{2}}
$$

where $D$ is the outer diameter of the tube and $D_{e}$ is an effective diameter of the tube array. The ratio $D / D_{e}$ is related to the pitch-to-diameter ratio $(P / D)$ of the tube array and is obtained empirically. Pettigrew et al. (1986, ref) provide empirical correlations for this ratio as shown in Table 3.2:

Table 3.2: Empirical expressions for $D_{e} / D$

\begin{tabular}{|c|c|}
\hline Pattern & Expression for $D_{e} / D$ \\
\hline Triangular & $1.7 \cdot P / D$ \\
\hline Square & $1.9 \cdot P / D$ \\
\hline
\end{tabular}


For the current test with a normal square array, the calculated value for the effective diameter ratio for a $P / D$ ratio of 1.4 is 2.66 , yielding a value of 1.175 for the multiplier for the confinement effect. The experimentally obtained mean value for the multiplier is 1.4 , which is about $19 \%$ higher than the theoretically expected value.

The damping ratio in single phase flow consists of the structural damping, s, and the fluid damping, f. The values in Table 3.2 and Figure 3.24 indicate the measured total damping, $\zeta=\zeta_{f}+\zeta_{s}$. The fluid damping component can be analytically expressed (Blevins, pp. 309) as

$$
\zeta_{f}=\frac{\pi}{2} \frac{\rho D^{2}}{m}\left(\frac{v}{\pi f D^{2}}\right)^{1 / 2}
$$

where is the fluid density, is the kinematic viscosity, $m$ is the mass per unit length of the tube including the effect of added mass, $f$ is the natural frequency of the tube, and $D$ is the diameter of the tube. $m$ is calculated as

$$
m=\frac{M}{b}+\frac{\rho}{4} \pi\left(D^{2}\right)
$$

The first term on the right hand side is due to the mass of the tube and the second term is the added mass term. The fluid damping obtained using the above expression is an approximation as it assumes small amplitude vibration, and a Stokes drag form for the drag coefficient. Higher order terms in the Stokes drag expansion are neglected. Since the fluid damping depends on the density and viscosity of the medium, it is negligible in air indicating that structural damping is the major component of the damping ratio. Theoretically, the total damping in water can be calculated by adding the fluid damping term in water to the structural damping obtained in air. For the experimental conditions with a single tube, the fluid damping in water using Equation 3.2 is 0.0021 implying a value for the total damping ratio in water of $0.011+0.0021=0.0131$. This value lies within the uncertainty of the measured damping ratio in water.

An important point to be noticed is that since the damping ratio of a tube in an array of flexible tubes is higher than a single flexible tube in a rigid array, the damping ratios measured in a fluid-elastic instability test with a fully flexible array is expected to be higher than that of a single flexible tube in a rigid array. This will be useful while plotting the results on the Connors' criterion map.

\subsubsection{Void fraction}

Void fraction measurements were made using the gamma densitometer. Prior to making measurements in the test section, a calibration run was carried out by calibrating the densitometer output with known static void fractions. The static void fractions were achieved by sequentially filling compartmented chambers with known volumes of water. The results are shown in Figure 3.25 and show good agreement between the theoretical known values and the calculated ones. The uncertainty in the void fraction measurements is estimated to be $\pm 4 \%$. 


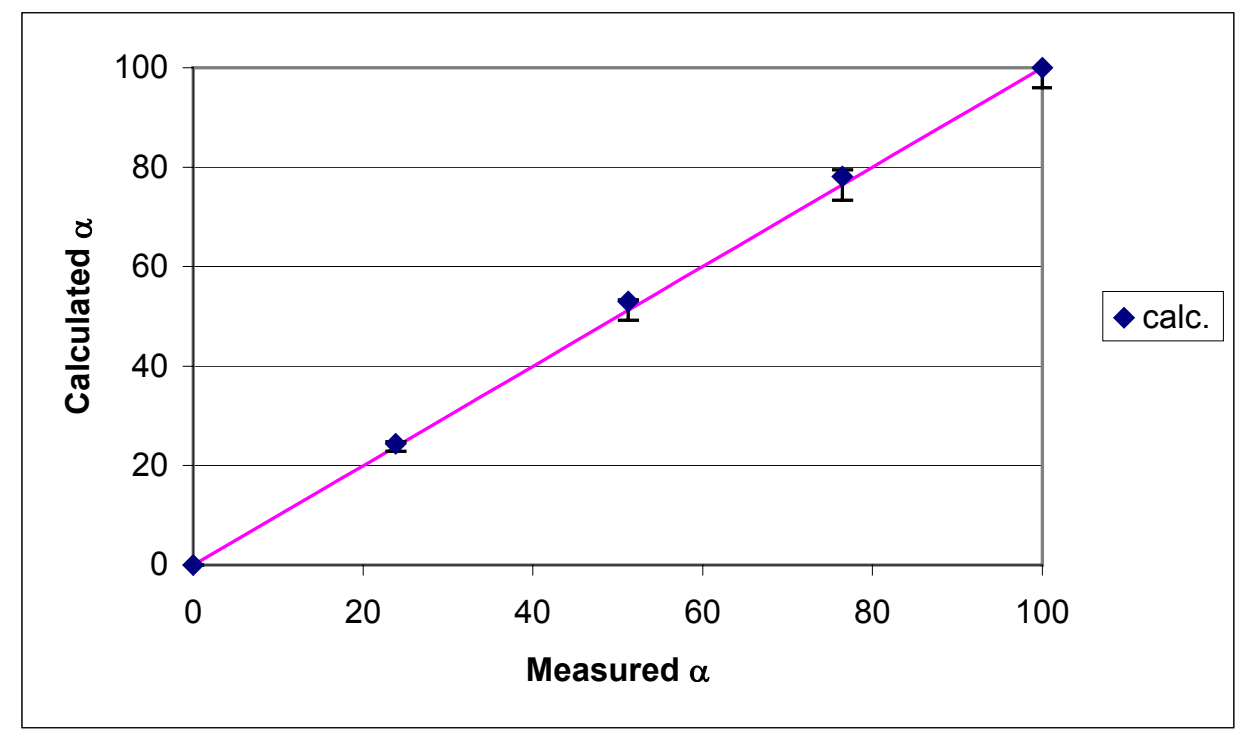

Figure 3.25: Gamma densitometer calibration test

Following this, the gamma densitometer was used to measure the void fraction of a flowing air-water mixture in the experimental test section. The gamma densitometer was placed on either sides of the viewports of the mixing section, approximately in the center and time averaged void fraction measurements were made by collecting the data for 30 seconds. (Insert new calibration curve). Each run was repeated thrice and the final value averaged over all three readings. The void fraction was controlled by setting the air flow rate at a constant value and increasing the water flow rates in steps. This was repeated for a number of air flow rates. Void fraction measurements were made for air flow and water flow rates in the range of 5-18.2 $\mathrm{cfm}$ and 10-60 gpm respectively. The results are presented in Figure 3.26.

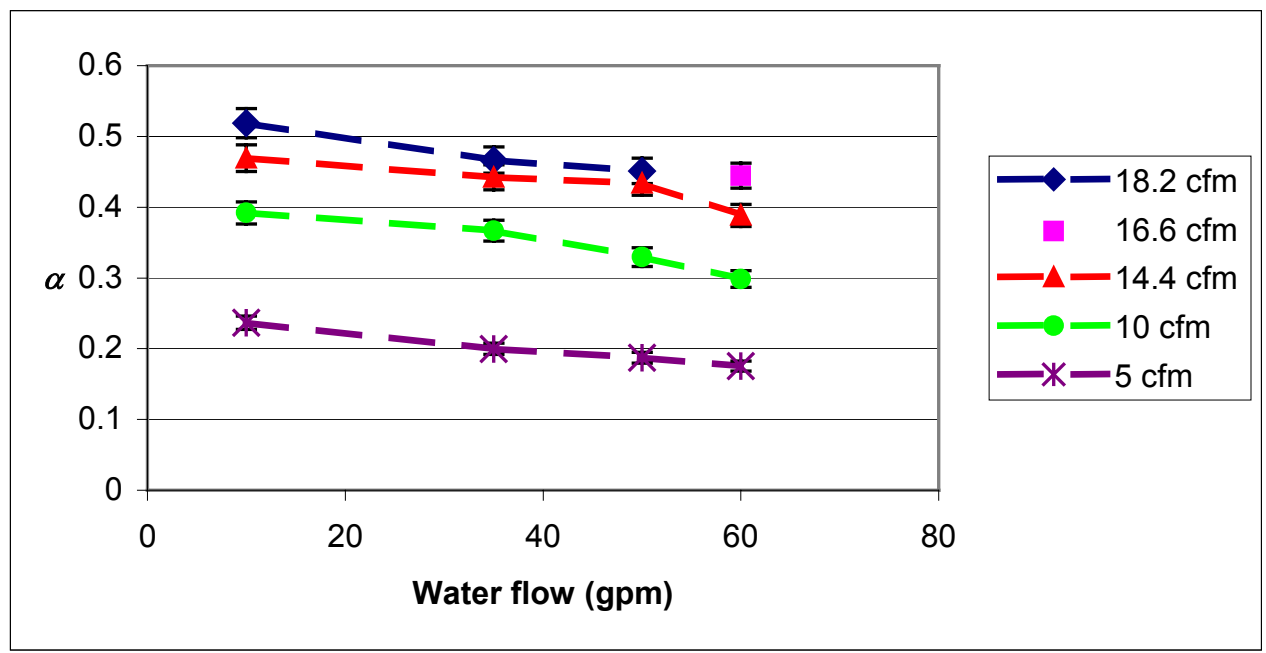

Figure 3.26: Air-water void fraction measurements made in experimental test-section 
The results indicate that the void fraction profile shows a weak dependence on the liquid flow rate. This was more pronounced at lower water flow rates less than $30 \mathrm{gpm}$. Hence, experiments with water flow rates less than $30 \mathrm{gpm}$ were not carried out during the fluidelastic instability runs. The results show that as expected, for a constant air flow rate, the void fraction decreases with an increase in the water flow rate. Also, for a particular water flow rate, the void fraction increases as the air flow rate is increased. In order to determine the validity of the results, the measured void fractions were compared with the Chexal-Lellouche Void Fraction Model for air-water flow in cocurrent upward vertical flow (Chexal et al., 1997). The analytical expression for the void fraction using this model is

$$
\langle\alpha\rangle=\frac{\left\langle j_{g}\right\rangle}{C_{O}\left\langle j_{f}+j_{g}\right\rangle+\bar{V}_{j g}}
$$

where $\alpha$ is the void fraction, $j_{g}$ and $j_{f}$ are superficial gas and liquid velocities based on the hydraulic area, $C_{o}$ is the concentration parameter, and $\bar{V}_{j g}$ is the drift velocity. $C_{o}$ and $\bar{V}_{j g}$ are fitted parameters. The code VOID4.EXE was used to calculate the void fractions knowing the superficial velocities and the temperature and pressure in the test section. The results for air flow rates of $5 \mathrm{cfm}$ and $14.4 \mathrm{cfm}$ at different water flow rates are shown in Figure 3.27.

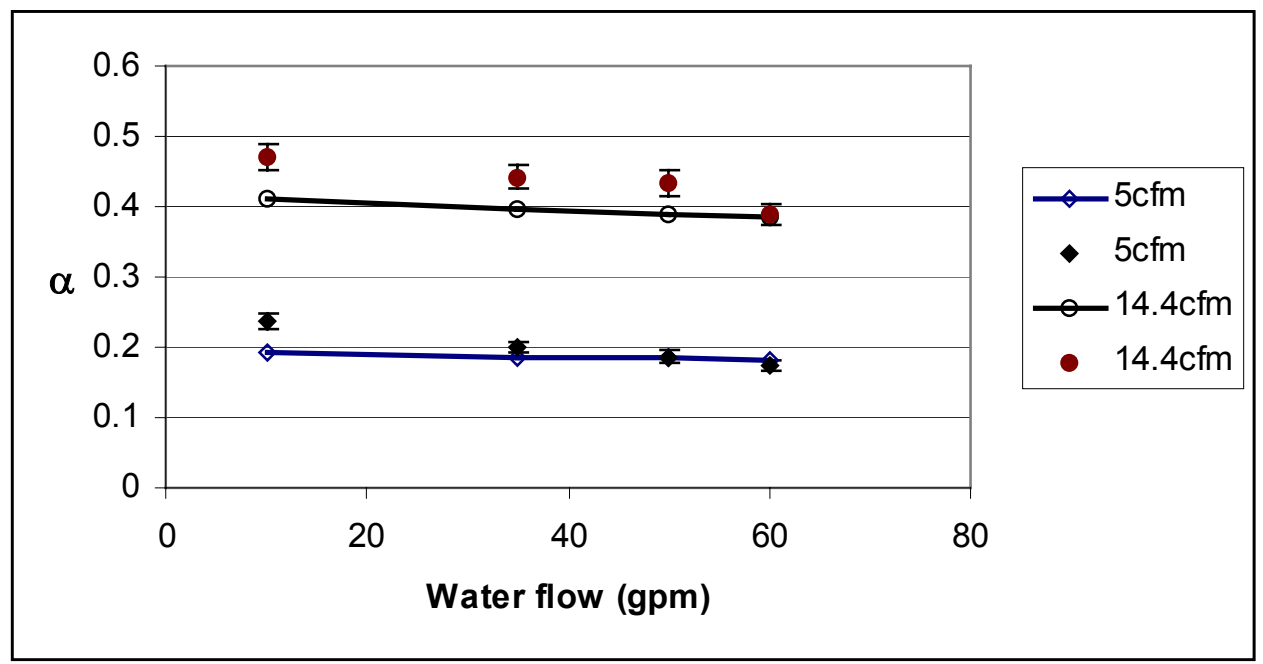

Figure 3.27: Comparison between experimental and theoretical void fractions

In the above figure, the solid symbols indicate the experimentally measured void fractions whereas the open symbols indicate the corresponding theoretically determined void fractions. It is seen that the values of the void fraction at an air flow rate of $5 \mathrm{cfm}$ match the correlation well. However, at high air flow rates, the agreement is not good with the measured void fraction consistently overpredicting the theoretically expected values except at the highest water flow rate tested. This is due to some amount of chugging in the viewing section, which increases the local void fraction measurements. The reason for the chugging is being investigated. 


\subsubsection{Fluid-elastic instability}

To demonstrate the occurrence of flow induced vibration, a single strain gage instrumented tube was installed in the test section. The first test for unstable vibration was carried out with water. The water flow rate was slowly increased and the strain gage signal noted. The vibrations remained very small and no instability was observed for a maximum flow rate of $70 \mathrm{gpm}$. The instability depends on the pitch velocity, which is the same as the free-stream velocity for a single tube. Since this value was very small, the instability did not occur. When an array of tubes is installed in the test section, the pitch velocity will be significantly larger due to the reduced flow area and instability is expected.

Next, an air-water mixture was introduced in the test section. The water flow rate was maintained constant at $32 \mathrm{gpm}$, and the air flow rate was slowly increased. At each step, the air flow rate was held constant for a period of time for which the strain gage signal was recorded. This was carried out for about six steps after which the large amplitude unstable vibrations were noticed. The time history signal of the strain gage signal as a function of the air flow rate is shown in Figure 3.28. The vibration of the tube can be made out from the amplitude of the strain gage signal. The dotted line between two time steps indicates the period when no data was recorded. The results are better interpreted if the root mean square (rms) value of the signal is calculated for each step and plotted as a function of the air flow rate at each step. This is shown in Figure 3.29.

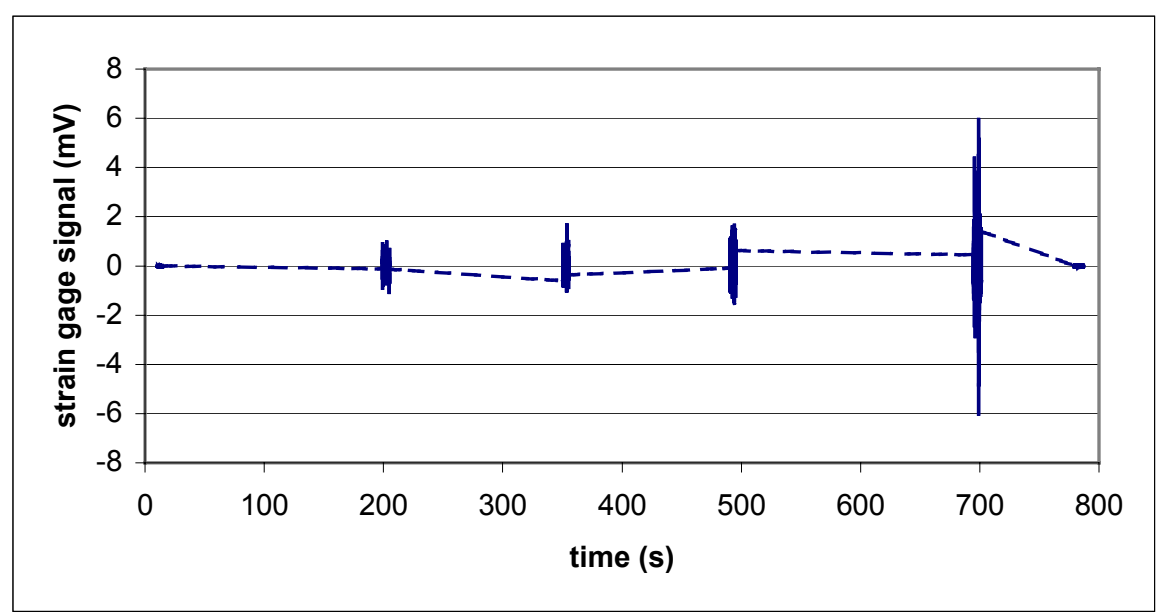

Figure 3.28: Time history signal of strain gage (Water flow- 32 gpm) 


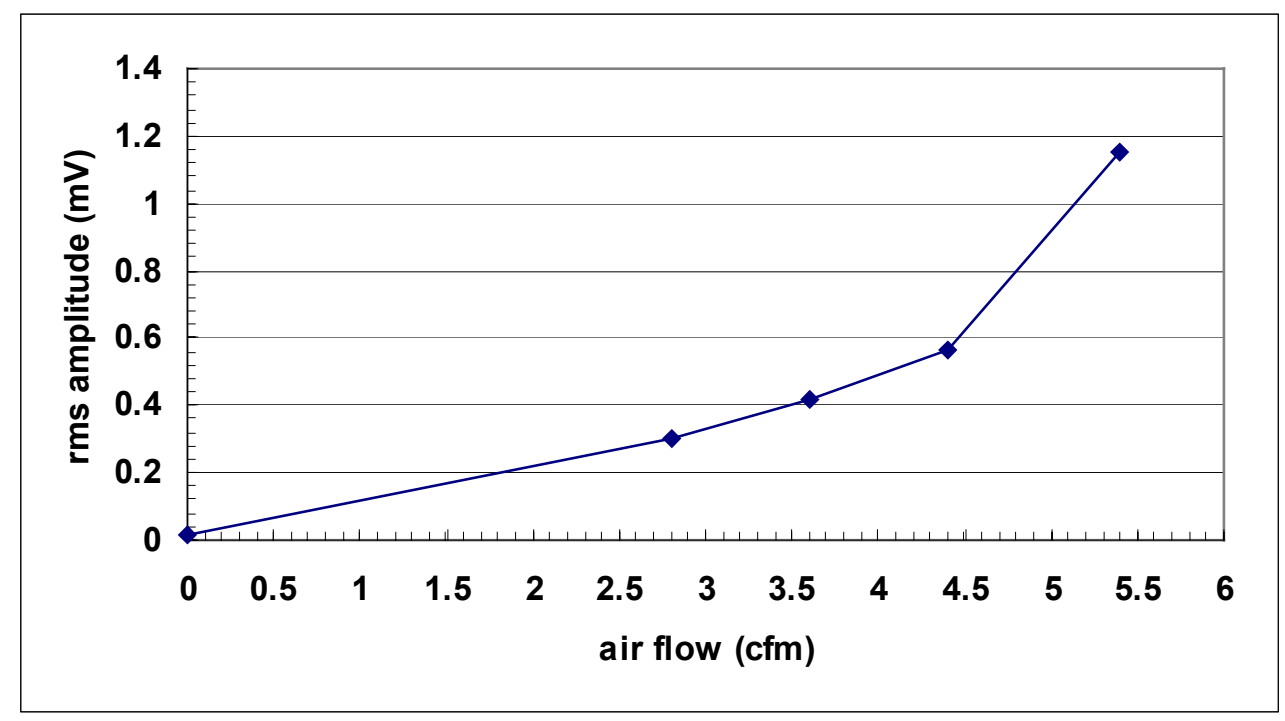

Figure 3.29: rms amplitude of strain gage signal (Water flow- 32 gpm)

The figure shows that the rms amplitude of the strain gage signal increases gradually with the air flow rate, and beyond a flow rate of $4.5 \mathrm{cfm}$, the amplitude increases to a very high value. A movie of the tube vibration was also made using a CCD camera. The movie shows almost no vibration in the direction of flow at zero flow rate. Vigorous vibration of the tube in an elliptical orbit can be seen at an air flow of $5.4 \mathrm{cfm}$. However, as reported in the literature, a sharp and sudden increase in the rms amplitude at the point of instability is not seen. Such behavior has been noticed by other researchers in the case of two-phase flow.

The preliminary results obtained in this chapter demonstrate the use of the experimental techniques to determine parameters to be measured at the onset of fluidelastic instability. They also set the stage for the actual full scale experiments to be performed which are described in the next chapter.

\subsubsection{Fluid-elastic instability experiments}

\subsubsection{Air-water flow}

Experiments were carried out with a fully flexible normal square array in airwater flow. for all the experiments, the strain gages were oriented such that their maximum sensitivity was in the direction of the flow (drag direction). The tubes were tuned to a frequency of $20 \pm 2{ }^{\circ} \mathrm{C}$ in air and their natural frequency decreased to $17 \pm 2{ }^{\circ} \mathrm{C}$ in still water. The first experiments were carried out with single phase water to determine the threshold of fluid-elastic instability for single phase flow. The flow rate of water was slowly increased in steps and the tube vibration was recorded. However, the maximum flow rate was limited by the pump to $70 \mathrm{gpm}$ corresponding to a liquid superficial velocity of $0.22 \mathrm{~m} / \mathrm{s}$. The vibration amplitude was steady for flow rates below $40 \mathrm{gpm}$ $\left(V_{l}=0.12 \mathrm{~m} / \mathrm{s}\right)$ and then gradually increased until a large increase in amplitude was observed at around $65 \mathrm{gpm}\left(V_{l}=0.20 \mathrm{~m} / \mathrm{s}\right)$. This point was identified as the critical flow 
rate required for instability. Fig 3.30 shows the root mean square amplitude of the strain gage signal of the central tube in terms of millivolts as a function of the liquid superficial velocity (flow rate).

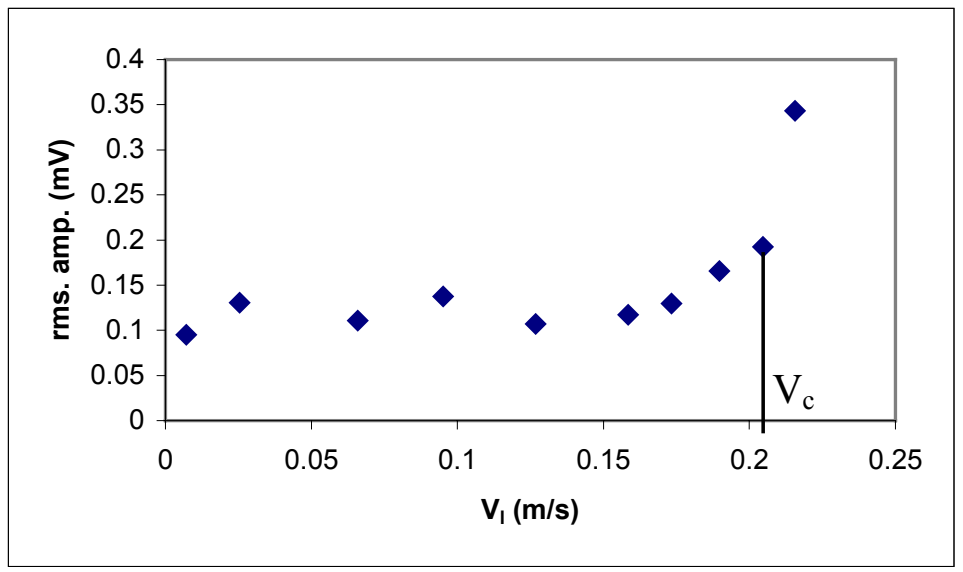

Fig 3.30 Tube vibration amplitude for single phase flow (t1_r2)

In subsequent air-water two-phase flow tests, the base liquid flow rate is held constant below the single-phase critical flow rate for an entire experimental run while the air flow rate is gradually increased.

Next, two-phase flow experiments were carried out for base water flow rates of 35,50 and $60 \mathrm{gpm}$ corresponding to superficial liquid velocities of $0.11,0.16$ and 0.19 $\mathrm{m} / \mathrm{s}$ respectively. In all these experiments, the water flow rate was held constant and the air flow rate was increased in steps and the vibrations recorded. The strain gage signals were post processed to determine the rms amplitude, frequency of vibration and damping ratio of the tubes. For the analysis, the unfiltered strain gauge signal is band passed through a digital filter and frequencies between $10 \mathrm{~Hz}$ and 30 to filter out noise. The choice of filter is selected knowing the expected natural frequency of vibration of the tubes. The mean value of the unfiltered signal for each air flow rate, which is proportional to the force acting on the tube, is determined. The root mean square value of the filtered signal is used to determine the dynamic vibration amplitude. Fig. 3.31 shows the time history of rms amplitudes and frequency spectrum of the strain gauge signal recorded for the central tube in the test with base water flow rate of $\mathrm{Q}_{1}=60 \mathrm{gpm}$. The time series plotted is the dynamic vibration amplitude obtained from the filtered signal (The horizontal axis displays the sample number, the sampling rate being $5 \mathrm{~ms}$; each frame shows 5 seconds of data). Some characteristics that can be easily observed are random large amplitude vibration at low flow velocities caused due to random vibration caused by the air bubbles hitting the tube. This is most easily seen in Fig 3.31(a) where the air velocity is almost zero (very few air bubbles present in the two-phase mixture). Fig 3.31(b) shows the vibration amplitude halfway between the instability. Fig 3.31(c) shows the vibrations at the onset of instability, whereas Fig 3.31(d) shows the post-critical vibration of the tube. 

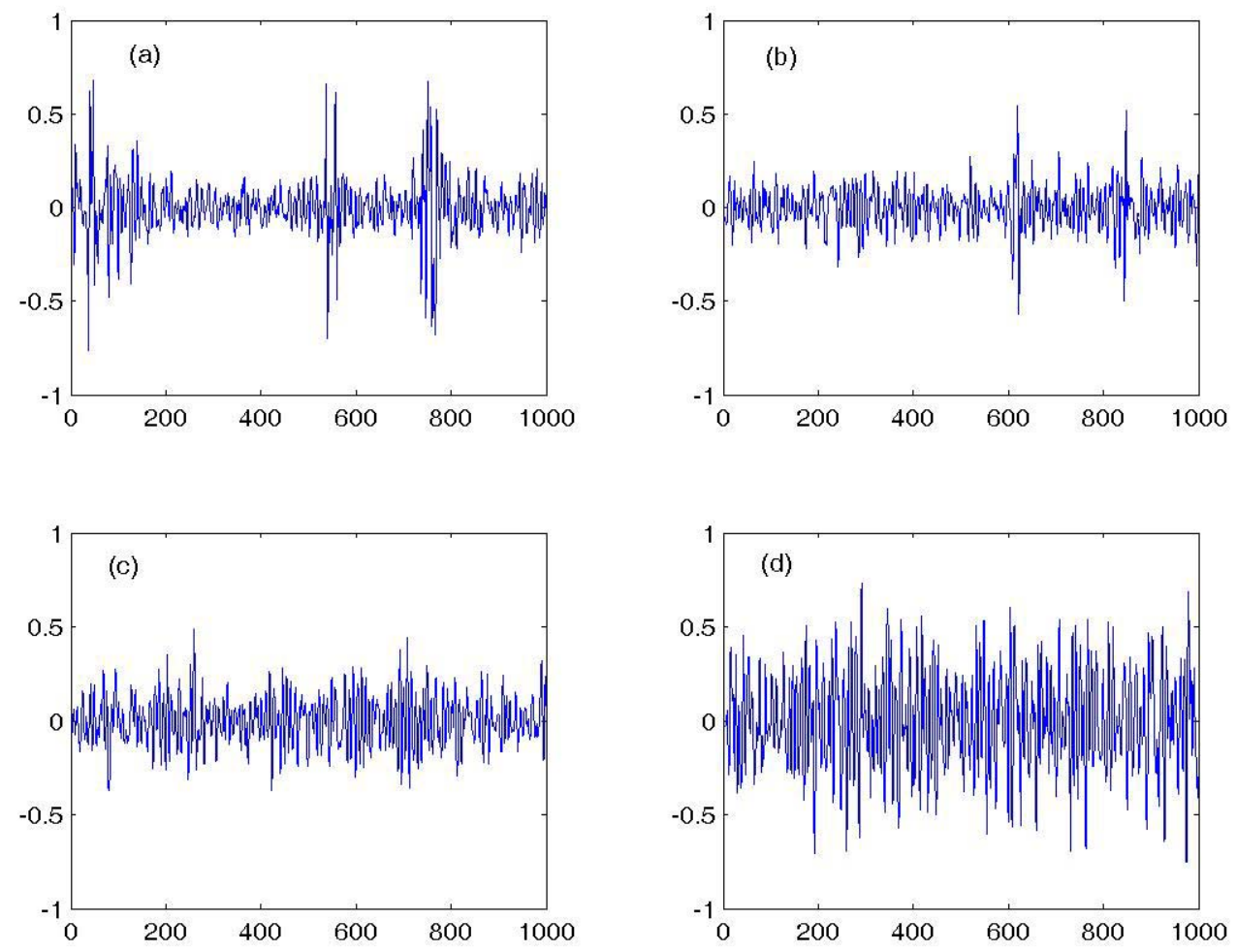

Fig 3.31 Strain gage signal of the central tube as a function of time, $\mathrm{Ql}=60 \mathrm{gpm}$; (a) $\mathrm{Vg}=0.03 \mathrm{~m} / \mathrm{s}$; (b) $\mathrm{Vg}=0.06 \mathrm{~m} / \mathrm{s} ;$ (c) $\mathrm{Vg}=0.08 \mathrm{~m} / \mathrm{s} ;$ (d) $\mathrm{Vg}=0.16 \mathrm{~m} / \mathrm{s}$

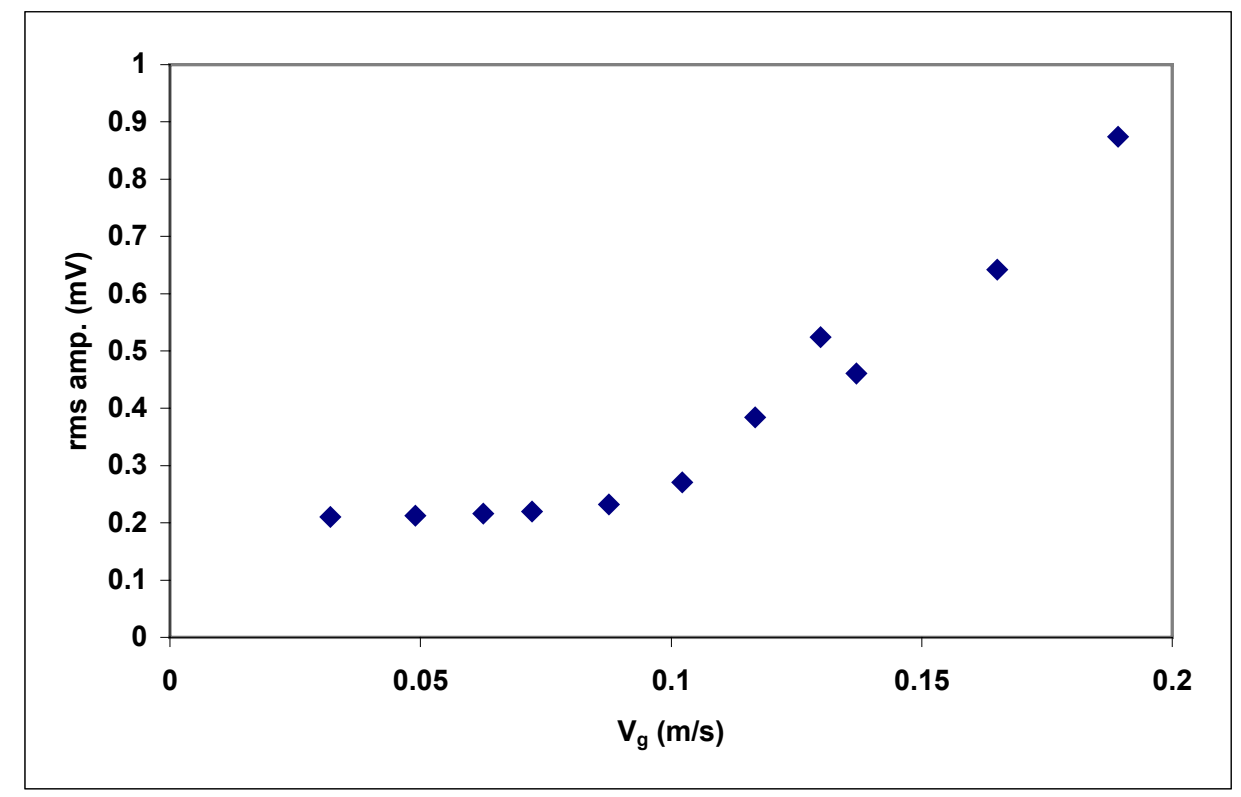

Fig. 3.32 Rms amplitude of strain gage signal as a function of air flow rate, $Q \mathrm{l}=60 \mathrm{gpm}$ 
This is more easily seen when the rms value of the amplitude of the strain gage signal is plotted as a function of the air velocity as shown in Fig3.32. The point where the slope of the rms amplitude changes determines the critical gas velocity required for instability. This is done by dividing the data into two regions, fitting linear curves through the two regions and determining the point of intersection of the two curves. This is demonstrated in Fig 3.33.

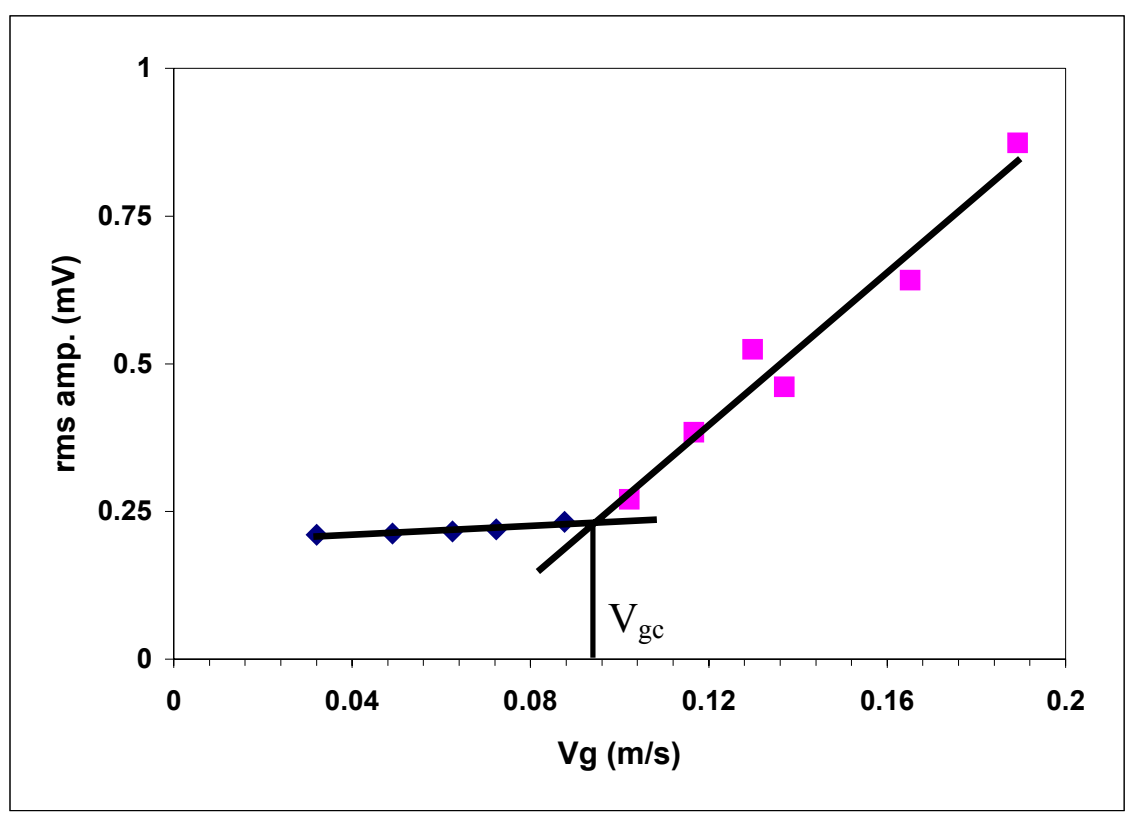

Fig 3.33 Demonstration of the determination of critical air velocity, $V_{\mathrm{gc}}$ for two phase flow runs $(\mathrm{Qg}=60 \mathrm{gpm})$

For this particular case, the critical air velocity is determined to be $0.09 \mathrm{~m} / \mathrm{s}$. From a similar plot, the critical void fraction is determined. The damping ratio is determined halfway between the point of instability following the convention used by previous researchers. It should be noted that the damping ratio measured in these tests is the total damping ratio of a single tube in a fully flexible tube array. This is more representative of the situation in a heat exchanger. Fig. 3.34 shows the amplitude spectrum of the strain gage signal at $\mathrm{Vg}=0.06 \mathrm{~m} / \mathrm{s}$. The natural frequency and the damping ratio were calculated from the spectrum to be $16.9 \mathrm{~Hz}$ and $12 \%$ respectively. 


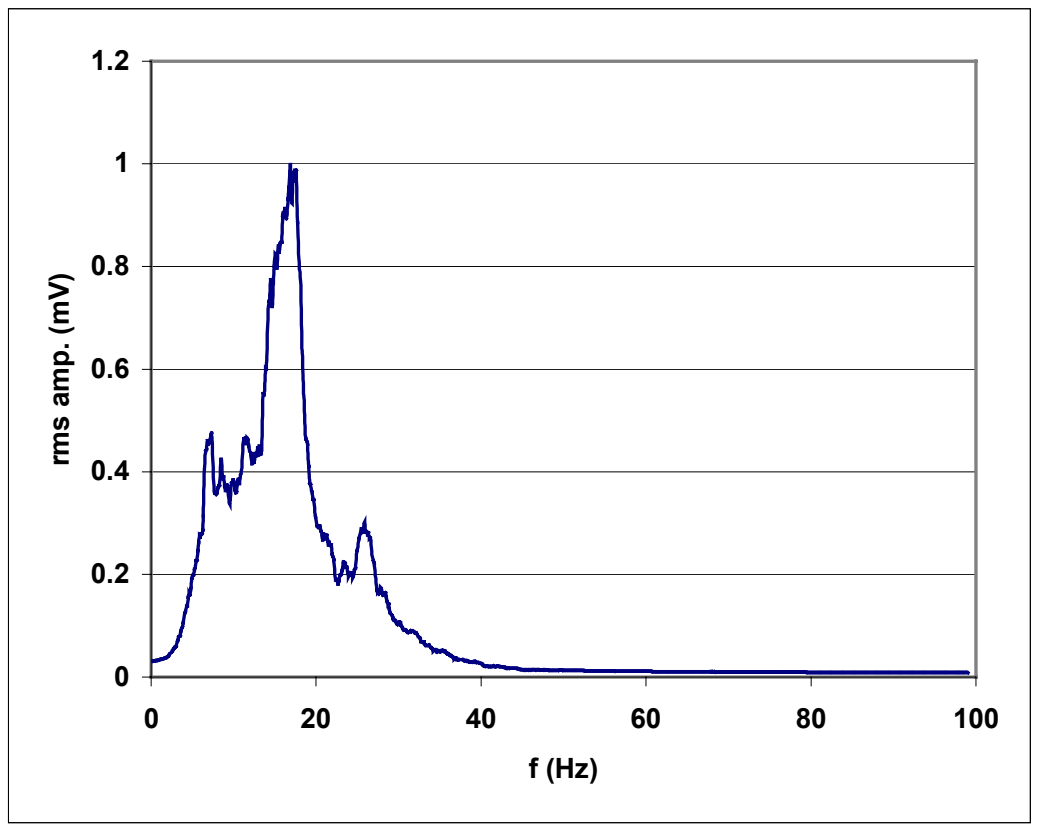

Fig. 3.34 Amplitude spectrum of the strain gage signal at $\mathrm{Vg}=0.06 \mathrm{~m} / \mathrm{s}$

Table 3.3 Parameters obtained at the onset of instability

\begin{tabular}{|l|l|l|l|}
\hline Parameter & \multicolumn{3}{|l|}{ Experimental Runs } \\
\hline$Q_{l}(\mathrm{gpm})$ & 35 & 50 & 60 \\
\hline$V_{l, \infty}(\mathrm{m} / \mathrm{s})$ & 0.11 & 0.16 & 0.18 \\
\hline$V_{c, p}(\mathrm{~m} / \mathrm{s})$ & 0.58 & 0.90 & 0.98 \\
\hline & 18.0 & 23.1 & 21.4 \\
\hline$\rho_{2 \phi}\left(\mathrm{kg} / \mathrm{m}^{3}\right)$ & 818.0 & 767.9 & 785.5 \\
\hline$f_{n}(\mathrm{~Hz})$ & 16.7 & 17.4 & 16.9 \\
\hline$\zeta(\%)$ & 6.0 & 7.0 & 12.0 \\
\hline$m(\mathrm{~kg} / \mathrm{m})$ & 0.89 & 0.87 & 0.88 \\
\hline$V c / f_{n} D$ & 2.2 & 3.27 & 3.64 \\
\hline $\begin{array}{l}2 \pi m \zeta \\
/ \rho_{2 \phi} D^{2}\end{array}$ & 1.62 & 1.98 & 3.34 \\
\hline
\end{tabular}

Table 3.3 summarizes the parameters obtained at the onset of instability for the three different tests. It should be noticed that the critical void fraction for instability for the case of $\mathrm{Vl}=0.11 \mathrm{~m} / \mathrm{s}(\mathrm{Ql}=35 \mathrm{gpm})$ is lower than those for $\mathrm{Vl}=0.16$ and $0.18 \mathrm{~m} / \mathrm{s}$. This was not expected since lower the water flow rate, the higher should be the amount of air to reach the level of unstable vibration. Repeats of tests at $V_{l}=0.11 \mathrm{~m} / \mathrm{s}$ showed the same results. Visually, it could be seen that the tube in this case was subject to a higher amount of random excitation by the air-water mixture, and this is believed to have caused an earlier instability. Occurrence of vortex shedding could also have led to this, but this was not verified in the experiments. 


\subsubsection{Fluid-elastic instability map for air-water two-phase flow}

The results of the two-phase flow experiments are plotted on the Connors' criterion plot in Fig 3.35. The figure also shows the results obtained by previous researchers and contains experiments carried out with air-water flow, steam-water flow as well as Freon-freon-vapor flow. The results from the three tests in the present study are plotted as closed circles.

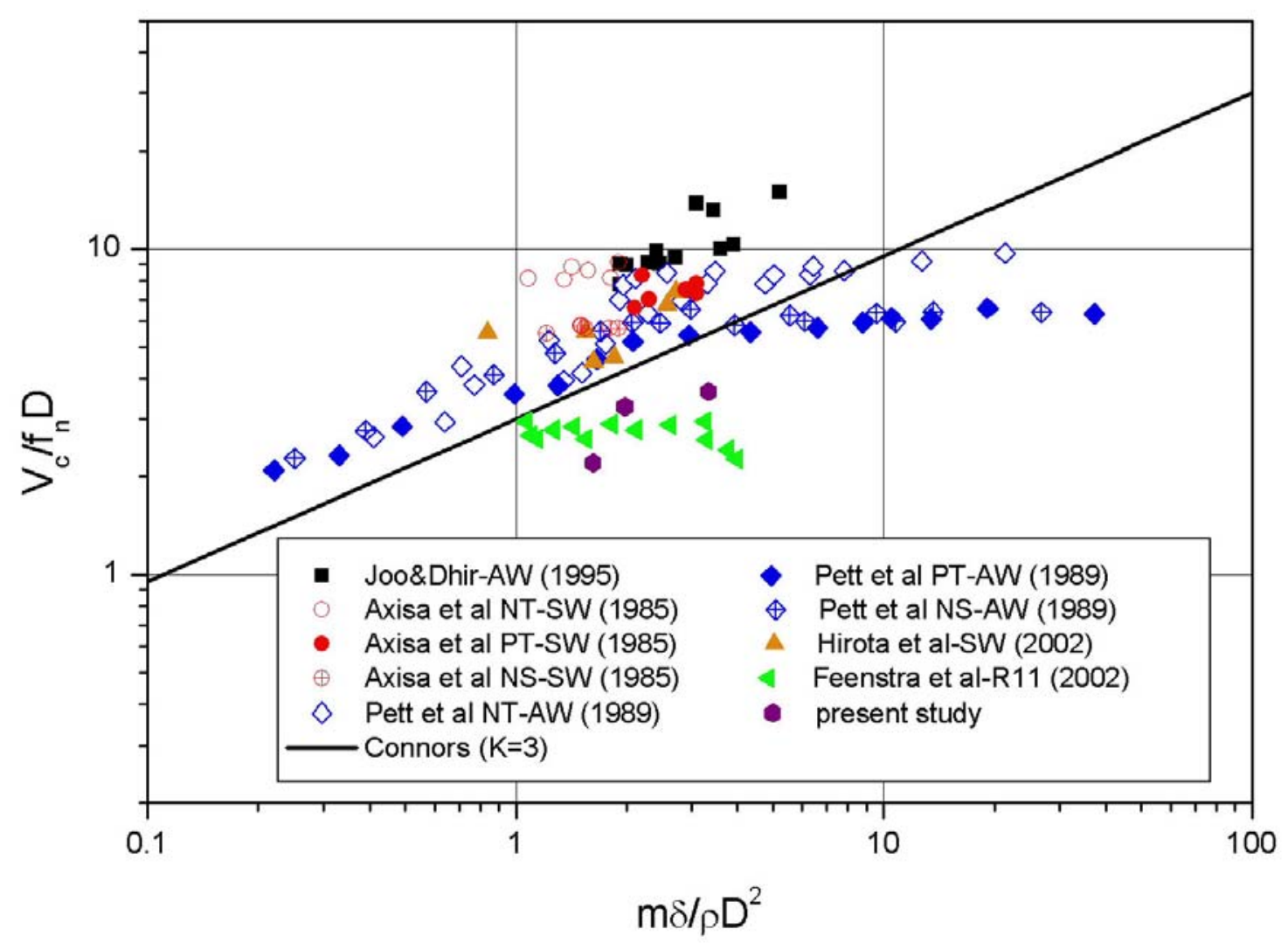

Fig 3.35 Connors' criterion plot

The figure also shows the widely adopted instability threshold standard obtaining by setting $\mathrm{K}=3$ in the Connors' criterion. The line indicates that for a given mass damping parameter, reduced velocities lying below the line are stable whereas those lying above the line are unstable. However, the present study shows that the instability threshold is lower than the prescribed criterion. Though surprising, this is attributed to the high mass damping ratios measured for the fully flexible bundle. The data in the above graph assume that the damping ratios are from measurements carried out for a single flexible tube in a rigid array, whereas the other parameters are measured in experiments carried out with a fully flexible array. Hence, experiments were carried out with a single flexible tube in a rigid array to determine the damping ratio. 


\subsubsection{Experiments with a single flexible tube in a rigid array:}

In order to construct a rigid array, tubes were jammed into clearance holes in the inner test section walls using Teflon inserts. The only flexible tube in the array was placed in the center of the fourth tube row and was the same as that for the fully flexible array tests. Once again, the natural frequency of the single tube was tuned to $20 \mathrm{~Hz}$ in air and it reduced to $17.5 \mathrm{~Hz}$ in water. Experiments were carried out for base water flow rates of $30,40,50$ and $60 \mathrm{gpm}$. The damping ratio measured for the test with $30 \mathrm{gpm}$ was used to approximate the value for that of $35 \mathrm{gpm}$ for the fully flexible array. The damping ratios measured for these tests are summarized in Table 3.4 and compared with those obtained from the fully flexible array test.

Table 3.4 Damping ratios measured in two types of tests

\begin{tabular}{|l|l|l|l|}
\hline \multirow{2}{*}{ Q1 (gpm) } & \multicolumn{2}{|c|}{$\mathrm{Vl}(\mathrm{m} / \mathrm{s})$} & \multicolumn{2}{c|}{ Damping ratio, (\%) } \\
\cline { 3 - 4 } & & Fully flexible array & Single flexible tube \\
\hline 30 & 0.09 & 6.0 & 3.5 \\
\hline 50 & 0.16 & 7.0 & 2.0 \\
\hline 60 & 0.18 & 12.0 & 3.4 \\
\hline
\end{tabular}

It is seen that the damping ratios measured in the case of the single flexible tube are much lower and almost same within the uncertainty of the measurement. The reduced velocity and mass damping parameters obtained in the fully flexible array experiments were recalculated using the damping ratios measured in the single flexible tube runs and the points were replotted on the Connors' criterion curve as shown in Fig 3.36.

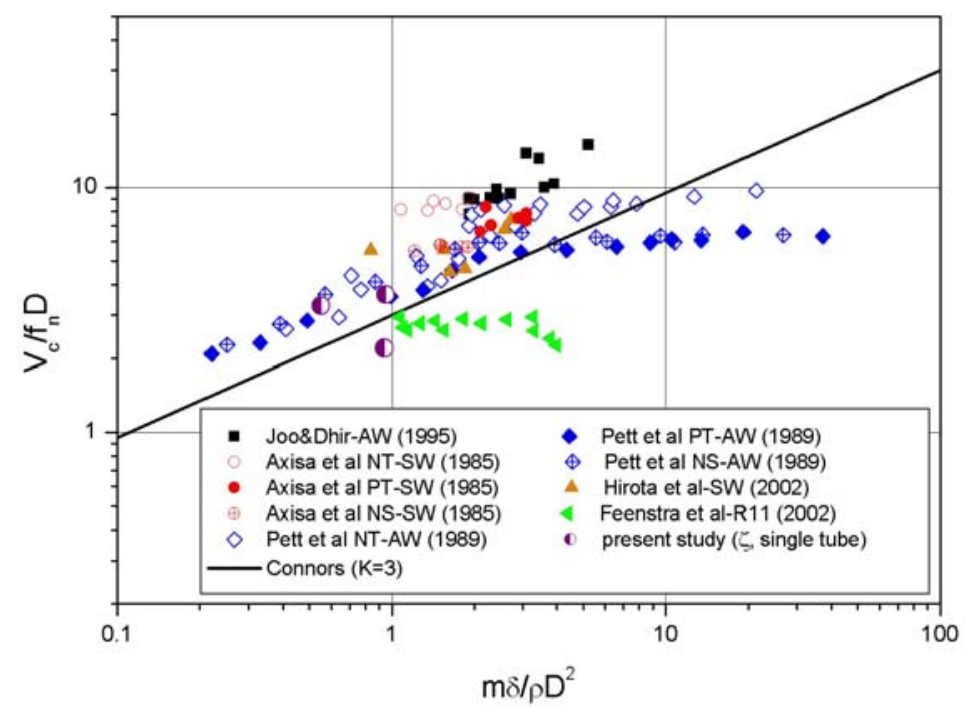

Fig 3.36 Replotted data

Replotting the data using the lower values of the damping ratio causes the data points to shift to the left causing the threshold value of instability to lie above the recommended value of $\mathrm{K}=3$. The point lying below the recommended threshold of instability is from the run for which $\mathrm{Ql}=35 \mathrm{gpm}$ and for which the instability was detected earlier. 


\subsubsection{Steam-water tests and elevated temperature air-water tests}

One of the main purposes of the two-phase flow experiments was to run experiments with steam-water two-phase flow. Most two-phase flow experiments are run on air-water systems. A few experiments (Feenstra et al, Pettigrew et al) have been carried out with Freon-freon-vapor flow. Very few experiments (Axisa et al, Hirota et al) have been carried out with steam-water flow to simulate exact heat exchanger conditions. The objective of the steam-water flow experiments in this study was not to run experiments at prototypical heat exchanger pressures and temperatures, but to run experiments at the same pressures as that of air-water flow ( 2 psig) and compare the results with those of the air-water flow experiments.

The steam-water flow experiments proved very tricky to run. The main hurdle was the mounting of the strain gages and their survival in the harsh steam-water environment. The gages were specially coated with two layers of protective coating (Measurements Group, M-Bond GA-55 and Dow Corning RTV 3145) to withstand the temperatures and the corrosive steam-water environment. Extensive tests were carried out to stabilize the strain-gage signal at high temperatures. In spite of using a half bridge circuit, which was expected to compensate for thermal expansion, the strain gages drifted with temperature, but eventually reached a steady value once the temperature reached a steady state. The other main drawback was the generation of a steam-water mixture. Since the steam flow rate produced by the electric boiler cycled with time, it was practically impossible to maintain a constant void fraction in the test section. This problem was circumvented by recording the tube vibration at the peak steam production period of the boiler cycle. Condensation of steam into the bulk water also proved a problem, especially for determining the steam flow rate in the test section. This occurred since steam had to be produced at a higher pressure at the source in order to overcome the pressure drop at the mixing section.

The experimental procedure adopted for conducting steam-water flow experiments is as follows:

- The bulk water is heated to just below the saturation temperature corresponding to the pressure in the test section by circulating through the loop at the desired flow rate. This step typically takes about 30 minutes.

- The steam flow rate is very slowly increased and the steam-water mixture is allowed to flow through the test section. The cycling of the void fraction is noted before recording the tube vibration at the peak void fraction.

- Void fraction of the mixture is measured using the gamma densitometer, which is placed across the vewports in the mixing section.

- This step is recorded for several steam flow rates (void fraction) until fluid-elastic instability is observed.

Fig 3.37 shows the results of a steam-water flow test in comparison to a similar test carried out with air-water flow. The steam-water flow test was carried out with a fully flexible normal square array at a base water flow rate of $20 \mathrm{gpm}\left(V_{l}=0.06 \mathrm{~m} / \mathrm{s}\right)$ whereas the air-water flow test was carried out at $30 \mathrm{gpm}\left(V_{l}=0.09 \mathrm{~m} / \mathrm{s}\right)$. As for the air-water flow 
case, the graph shows the rms amplitude of the tube vibration as a function of the upstream free stream void fraction. In the steam-water flow cases, the void fraction which is measured directly is plotted on the horizontal axis. This is done since the steam velocity cannot be measured accurately, as opposed to the air-water flow experiments where the amplitudes were plotted as a function of air velocity. The result shows that for similar base water flow rates, the tubes become unstable at a much lower void fraction for steam-water flow. On visually observing the flow pattern, it is noticed that the void fraction seems to increase as the steam-water mixture flows through the tube bundle. To an extent, this is expected due to the reduced flow area available for the two-phase flow through the tube bundle. But this effect is more pronounced for the case of steam-water flow than for air-water flow. This effect has not been fully investigated but possible reasons could be the change of pressure and temperature along the test section, subcooling of the bulk fluid which might cause the water to gain energy from the steam eventually reaching saturation and hence flashing as the flow passes through the tube bundle.

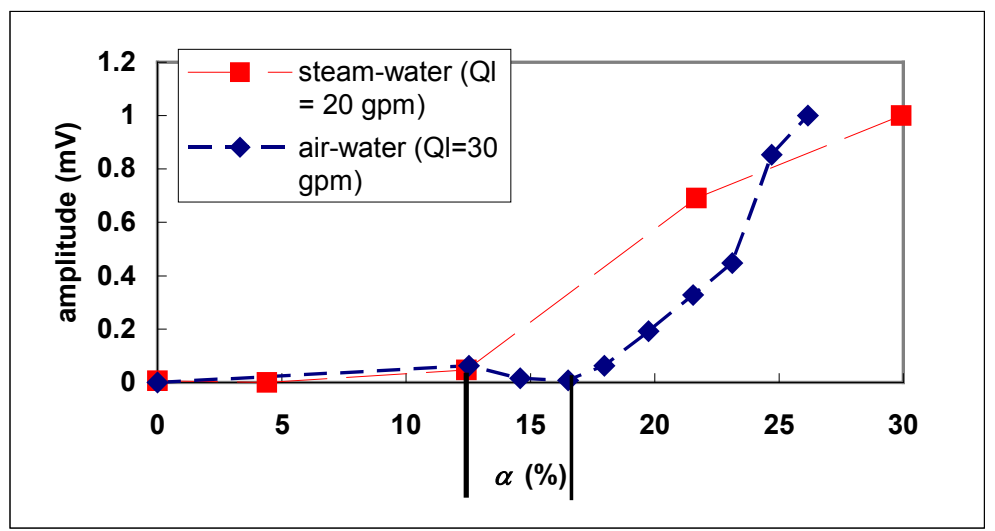

Fig 3.37 Comparison of steam-water flow tube vibration amplitude with air-water flow

In order to understand this effect in a better manner, experiments were proposed with air-water flow at near saturation temperatures.

\subsubsection{Air-water flow experiments at elevated temperatures}

These sets of experiments were proposed since problems such as cycling of void fraction, condensation of the vapor phase or bulk heating of the liquid could be avoided. Moreover, air-water flow experiments are easier to run than steam-water flow experiments. In all these experiments, a single flexible tube in a rigid normal square array was used. In these experiments, the bulk water was heated to $99^{\circ} \mathrm{C}$ and air at room temperature was introduced into the air line. Since the air was at room temperature, the air expanded as it reached the higher bulk water temperature to form the two-phase mixture. The results from tests carried out at base water flow rates of 35, 50 and $60 \mathrm{gpm}$ are shown in Fig 3.38 along with the results from the air-water flow tests. As in the case of the steam-water flow experiments, it is seen that the tubes become unstable at a very low void fraction. This was very surprising since the void fractions needed to achieve instability were lower than that for steam-water flow and were almost independent of the 
liquid flow rate. Once again, it is visually observed that the void fraction appears to increase as the two-phase flow passes through the tube bundle. In order to understand the differences between the three fluid systems, the relevant fluid properties were determined and are shown in Table 3.5 below.

Table 3.5: Relevant Fluid Properties

\begin{tabular}{|l|l|l|l|}
\hline Property & Air-water $\left(30^{\circ} \mathrm{C}\right)$ & \multicolumn{1}{|c|}{$\begin{array}{c}\text { Air-water } \\
\left(100^{\circ} \mathrm{C}\right)\end{array}$} & \multicolumn{1}{|c|}{$\begin{array}{c}\text { Steam-water } \\
\left(\mathrm{P}_{\text {sat }}=2 \mathrm{psig}\right)\end{array}$} \\
\hline$l\left(\mathrm{~kg} / \mathrm{m}^{3}\right)$ & 996.2 & 958.5 & 958.5 \\
\hline$v\left(\mathrm{~kg} / \mathrm{m}^{3}\right)$ & 1.16 & 0.95 & 0.60 \\
\hline$l(\mathrm{~Pa}-\mathrm{s})$ & $8.0 \mathrm{e}-4$ & $2.82 \mathrm{e}-4$ & $2.82 \mathrm{e}-4$ \\
\hline$v(\mathrm{~Pa}-\mathrm{s})$ & $1.87 \mathrm{e}-5$ & $2.18 \mathrm{e}-5$ & $1.23 \mathrm{e}-5$ \\
\hline$l v$ & 858.79 & 1008.9 & 1597.5 \\
\hline$l^{\prime}$ & 42.78 & 12.94 & 22.92 \\
\hline
\end{tabular}

The large difference in viscosity ratios for the fluid systems is easily noticed. This could lead to a difference in slip velocities causing steam or air at higher temperatures to flow faster than at lower temperatures causing the fluid force acting on the tube to be different in the two cases. Clearly, differences exist between the two systems and more systematic experiments are needed to resolve them.

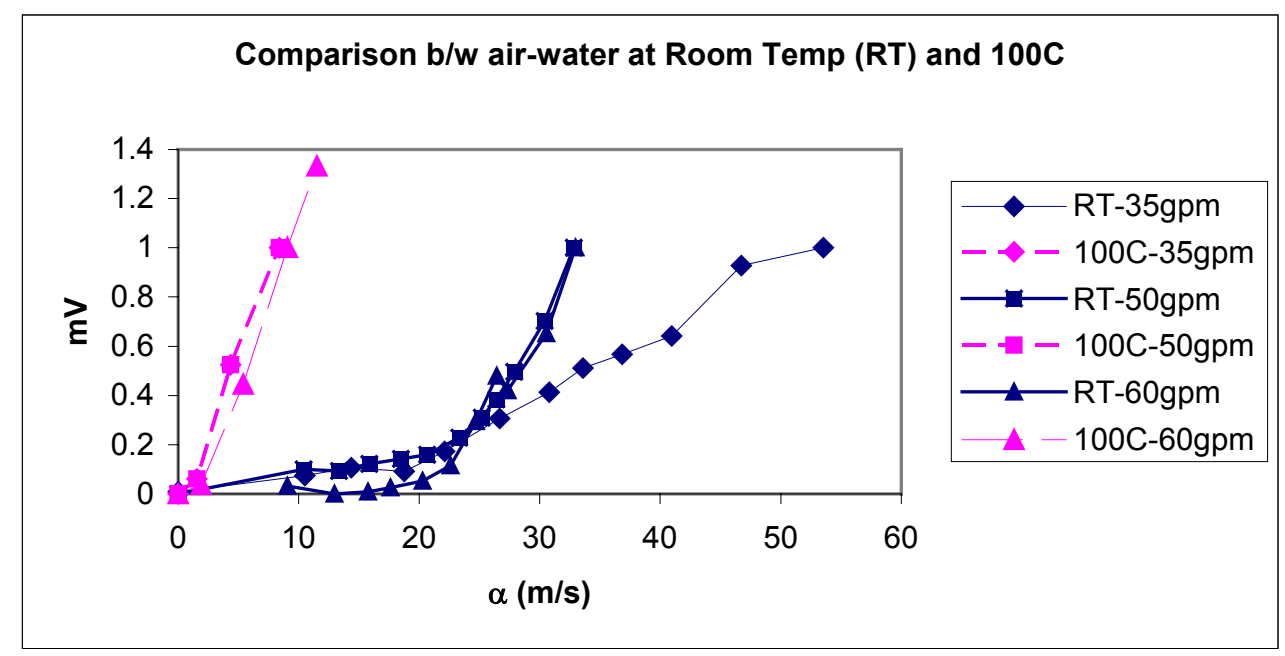

Fig. 3.38 Air-water flow experiments at elevated temperatures 


\section{Project Performance}

\subsection{Single Phase Experiment:}

1. Observe and quantify the real-time displacements of each tube in the bundle.

The time-dependent displacements of a normal square configuration and a normal triangular configuration of tube arrays have been observed and quantified for a large range of flow speeds, including conditions under which vortex shedding and fluid-elastic instability phenomena occurred. Furthermore, several methods of analysis of the tube vibration data have been developed and successfully applied to the current experimental data set. The single-phase experimental apparatus developed towards this goal may be used to further investigate various tube array geometries of interest including different tube patterns and pitch-to-diameter ratios.

2. Observe and quantify the instantaneous velocity field surrounding the tube bundle.

Measurement of the instantaneous velocity field surrounding the tube bundle was not achieved in this study. Directly measuring the local velocity within the tube bundle using intrusive methods such as hot wire anemometry or pitot devices was impossible due to the nature of the experiment; the proposed methods included the use of optical techniques like particle image velocimetry (PIV) or laser Doppler velocimetry (LDV). Although such methods are applicable and most likely optimal for the single phase experiment, several obstacles, including the procurement of expensive optical equipment, requirement of flow seeding, very high speed cameras, and the inability to use the same method for the two phase flow experiment, prevented the implementation of optical measurement systems within the time frame of the current study. However, the data obtained from the current experiment clearly indicates that the structural response of the tube bundle depends strongly on the general flow regime, as well as on the structural configuration of the tube bundle itself. Thus, while the exact velocity field has yet to be determined, several design recommendations and conclusions may be inferred from the data obtained so far (see Chapter 6).

\subsection{Two Phase Experiment:}

1. Revisit the problem of fluid-elastic instability for two-phase steam-water and airwater flow and conduct systematic experiments to determine experimental parameters at the onset of instability: the flow velocity, frequency of vibration of the tubes, damping ratio of the tubes, and the void fraction of the mixture.

Experiments were carried out with air-water flow at room temperature and $96^{\circ} \mathrm{C}$ and with steam-water flow. The parameters mentioned above were measured and a Connors' criterion plot was made based on these results. The experiments were 
carried out with stainless steel tubes arranged in a normal square array of pitch-todiameter ratio of 1.4. The two-phase flow loop used for this research will be used in the near future to further investigate fluid-elastic instability in steam-water flow.

2. The effect of pitch-to-diameter ratio on the instability will be investigated by changing the pitch of the tube array to obtain $P / D$ ratios of 1.4 and 1.55 for the normal square array.

Due to the limitations of the flow capacity of the flow loop, additional pitch-todiameter ratios were not investigated. This was because a higher pitch-to-diameter ratio requires a higher flow velocity for inducing instability and this could not be achieved with the installed pump in the flow loop.

3. Study the effect of nucleate boiling on the tube surface in steam water flow

This study was not undertaken due to the difficulties encountered in observing instability in steam-water flow. Based on the experience of the steam-water flow experiments, it is necessary to replace the installed pump with a higher capacity pump and carry out the steam-water flow experiments with the base liquid flow rate near the single phase flow instability threshold. This ensures that the twophase flow is uniform and that vibrations due to random excitation are minimized. Based on the current experimental setup, experiments with nucleate boiling on the tube surface are not expected to yield results any different from those observed with steam-water flow.

4. Limited study of the circumferential and radial void fraction distribution around a rigidly held tube in a normal square tube array in steam-water flow.

This study was not carried out due to the difficulty of measuring void fractions in the small area around the tubes with the gamma densitometer. This was again attributed to the flow characteristics of the steam-water flow at the liquid flow rates tested.

5. Determine the pressure distribution around a rigid tube instrumented with pressure taps around its periphery in steam-water flow.

Inability to develop the instrumentation for measuring the pressure around the periphery of the tube in steam-water flow prevented this study to carried out. Nevertheless, this work would yield important results that could be used to understand the nature of fluid-elastic instability and the differences between steam-water and air-water flow systems. This work will be pursued in the near future in order to draw a comparison with the air-water flow pressure data that already exists from previous research. 


\section{Project Publications, Presentations, and Technology Transfer}

The following list of items includes all of the various sources of information concerning the project, with brief details about the contents of each. The items are given in chronological order by date of publication or presentation.

Alquaddoomi, O., Catton, I., Adinolfi, P., "Methods of Analysis of tube array Fluid elastic instability data", Proceedings of IMECE'03 2003 ASME International Mechanical Engineering Congress \& Exposition, Washington, D.C., November 16-21, 2003

Adinolfi, P., Alquaddoomi, O., Catton, I., and Dhir, V.K., Fluid Elastic Instability of a tube array in water cross-flow, paper submitted to the Symposium on Flow-induced Vibration, ASME Pressure Vessels and Piping Conference 2003, July 20-25

Catton I., Adinolfi, P., Alquaddoomi, O., "Fluid-Elastic Instability in Tube-Arrays", Transactions of the American Nuclear Society: Winter Annual Meeting 2002, Washington D. C., November

Mitra, D., Adinolfi, P., and Alquaddoomi, O., "Development of an Experimental Apparatus for Studying Fluid-Elastic Instability," Transactions of the American Nuclear Society: Summer Annual Meeting 2002, Florida 


\section{Conclusions and Future Work}

\subsection{Single-Phase Experiment}

\subsubsection{Conclusions}

For the purpose of control of fluid-elastic instability, a general criterion for the design of single-phase heat exchanger tube bundles may be inferred utilizing the results obtained through this experimental investigation. It is immediately apparent that the onset of FEI is somewhat ambiguous, both because the process is gradual at the initial stages of onset and differs between different geometric and structural (natural frequency of tube array) configurations. The amplitude of vibration slowly increases as the flow velocity increases and at some point grows very quickly. Any attempt to define a specific critical velocity at which FEI occurs will necessarily be a function of what amplitude is defined as the threshold of instability; this naturally depends on the configurations mentioned above. However, general observations that are more useful than the exact critical velocities may be derived here, especially regarding the overall response of the tube array to the imposed changes in configurations and how they may improve the performance of a given tube bundle design or help to choose between competing design proposals.

\section{Square array}

From the results presented in section 3, it is clear that a normal square array configuration is far more susceptible to damaging FEI vibrations than a triangular array configuration over a comparable range of operating velocities. Furthermore, the onset of FEI is much more sudden and violent in the normal square case and, hence, significantly more difficult to predict and prevent. The only square array configuration which did not experience such an onset was the highest frequency of tuning, i.e. the $20 \mathrm{~Hz}$ case. A preliminary conclusion that may be made concerning the normal square array configuration is that the normal square array design must provide a sufficiently high stiffness at the tube supports to avoid a catastrophic onset of FEI.

\section{Triangular array}

The onset of FEI for the normal triangular array configurations varies significantly for each of the different tube frequency tunings. However, the critical velocities of each tuning case for the triangular arrays are greater than those measured for the respective values in the normal square array pattern. Thus, the triangular array is the better design option for the prevention of FEI until higher reduced velocities are attained, resulting in a more favorable economic performance as compared to the square array. Observations of the tube motion for the triangular configuration show a very complex pattern emerging. The pattern appears to be almost statistical in nature with the tubes seeming to behave more independently of one another than the square array, indicating that tube-to-tube coupling may have a reduced influence on the tube vibrations. Furthermore, it is clear that, for the normal triangular array, the vibration amplitude 
varies with respect to the spatial location of each tube considered. In particular, the magnitudes of the displacements within the array increase towards the downstream flow region. The reasons for this are not apparent, requiring more knowledge of the exact velocity field, but demonstrate that one should use data from single oscillating tube experiments with caution.

\section{Vortex shedding}

A large amount of data has been collected concerning the vortex-shedding phenomena and its influence on the vibration of the tube arrays under consideration. This data directly provides information for the characterization of the vortex-shedding induced vibration based on the various parameters of the study. Additionally, it allows for the investigation of the possible influence of vortex shedding from the tubes on the onset of FEI, since the vortex shedding phenomena typically occurs at a reduced velocity near that of the FEI onset.

The occurrence of the vortex shedding provides a reference point for the vibration amplitude, both in terms of magnitude and the location of the vortex induced vibration peak. Observation of the maximum vibration amplitude associated with vortex shedding provided a mark for identifying the onset of instability: the threshold of the instability amplitude is required to be relatively greater than the vortex shedding peak amplitude. The energy associated with the vortex shedding is observed to change significantly as a function of the tube array natural frequency, indicating that the fluid-structure interaction depends heavily on the tuning of the tubes, as suspected. The vortex shedding allows for the measurement of the Strouhal frequency, which may be related (using the frequency spectrums of the tube vibrations) to the natural frequency of the tubes, providing an insitu method of verification of the tube tuning. In these respects, observation of the vibrations induced by vortex shedding provide valuable information relevant to the entire scope of the experiment.

The reduced velocity range over which the vortex shedding mechanism occurs is also a rough indication of the flow field and streamline patterns. In some cases the occurrence of the vortex shedding peak coincides with the onset of the FEI and may indicate a triggering mechanism in this case, as well as an additional energy source for vibration when coupled with the FEI. The reduced velocity range over which the vortex phenomena is prevalent seems to be a primary difference between the FEI onset for the square and triangular configurations

\subsubsection{Future work}

In order to gain a better understanding of the physics of the occurrence of fluid elastic instability is necessary to resolve the flow field around the tubes, to accurately identify the forces acting upon the cylinders. This effort would lead to a complete description of the phenomenon, making possible the development of semi-analytical models. Different techniques have been considered to investigate the flow field. Currently the most promising method appears to be the Particle Image Velocimetry 
(PIV). This technique basically consists of illuminating the flow, with a laser light sheet, at a specific plane perpendicular to the axis of the tubes, and then capturing a pair of images of the flow field at two instants in time; the flow field is visualized by seeding the flow with reflective particles that light up as they pass through the plane of illumination. When a double-exposure image is taken, it may be processed to retrieve the full twodimensional velocity field about the entire viewable tube bundle at a given instant in time. Given the setup of the experiment described in this study, the method emerges as very convenient. In fact, the digital camera already in use is suitable for this kind of technique and the test section has been designed as to be optically accessible. Once the velocity field immediately surrounding the tubes is resolved, it will be possible to measure the exact pressure distribution on the tube surface, as well as to investigate the flow structures evolving around the onset of FEI.

Other alternatives exist for measurement of the pressure distribution, and hence the fluid forcing on the tubes. Pressure taps may be installed along the circumference of each of the tubes at various axial positions, providing an approximate measurement of the pressure distribution around each tube. However, this method depends heavily on the accuracy of the pressure measurement devices due to the high fluctuations in velocity (highly turbulent flow), thus requiring expensive, high response time measurement systems. Also, such a technique is prohibitively intrusive, requiring either mechanical or electrical probes to be fed into the test section, adding a great deal of complexity to the experimental design. It seems that the best approach, used successfully in this project, would be to apply optical inspection methods to measure the velocity and pressure.

There remain very many configurations and possible changes in design parameters for which experimental data is needed for the analysis of FEI in tube array heat exchangers. One such parameter is the pitch-to-diameter ratio of the array (held constant in this experiment at a value of 1.4). It is predicted, both analytically and from experimental observation of FEI onset, that the pitch-to-diameter ratio has a profound influence on tube-to-tube coupling, and hence on the mechanisms directly related to all types of fluid-induced vibration. Hence, this parameter is of primary importance and should be a continuing focus of experimental work.

Finally, several other types of fundamental tube array geometries remain to be tested using the methods outlined here. Two of the major configurations were used in this study, but data and analysis on many other geometries exist, such as rotated square and rotated triangular configurations. Experiments on different array geometries would be simple to implement into the current setup, requiring only that the appropriate test sections are constructed and installed.

\subsection{Two-Phase Experiment}

\subsubsection{Conclusions}

The two-phase flow experiments were carried out with the aim of pointing out differences between different two-phase fluid systems used. Since most experiments in 
two-phase flow induced vibration have been carried out with two-phase flow and a growing body of literature points at the importance of determining the effect of flow regime and void fraction profiles, it was appropriate to carry out experiments with different two-phase flow systems.

To this effect, a two-phase flow loop was constructed in the Boiling Heat Transfer Laboratory at UCLA. This flow loop is capable of generating air-water and steam-water mixtures and is also designed to handle high-pressure steam-water flow. The fluid-elastic instability experiments were carried out on a fully flexible normal square array as well as a single flexible tube in a rigid array. The experiments were limited to a narrow range of flow rates limited by the capabilities of the liquid centrifugal pump. This led to the results being limited to a narrow range of mass damping parameters and reduced velocities.

Within the time frame of the project, experiments were carried out with a normal square array in air-water flow, steam-water flow and elevated temperature air-water flow. The latter set of experiments has not been performed by other investigators and shows a difference from room temperature tests of air-water flow systems. This is useful given the ease of performing elevated temperature air-water systems. Experiments carried out with the three systems show that fluid-elastic instability is observed at a lower void fraction for steam-water and elevated temperature air-water systems as compared to room temperature air-water tests.

Fluid-elastic instability maps have been developed for the present tests and compared with data of other researchers for air-water flow. An attempt is made to explain the difference between the different two-phase fluid systems using the viscosities of the two phases.

\subsubsection{Future work}

The differences obtained between the two fluid systems indicates a need to closely investigate factors such as flow regime, void fraction around a tube and the pressure distribution surrounding a test tube. The limited time frame of the project prevented the investigation of these factors. An important factor to study is the relation between the free stream void fraction and the void fraction within the tube bundle.

Pressure distributions around a test tube can be carried out by instrumentation of the test tube with pressure probes and study the pressure profile. The results of such tests will help in determining the validity of air-water tests and in explaining differences between the fluid systems used in the experiments.

Finally, differences in structural parameters, especially the pitch-to-diameter ratio and more importantly, tube array pattern should be investigated. This might turn out to be a parameter that influences the void fraction and flow regime within a tube bundle thus affecting fluid-elastic instability. 


\section{References}

Mam, I., Catton, I., "Flow-Induced Vibration Problem in a Simple Cylinder Array - One Dimensional Unsteady Integral Approach", 1990 ASME Winter Annual Meeting, F'EDVol.102, Dallas TY.., 1990

Mam, L, Catton, I., "Flow Induced Vibrations in Cylindrical Bundles: Two Dimensional Analysis into Normal Modes", Proc. 1991 National Heat Transfer Conference, Vol. HTD-165, Minneapolis, Minnesota, 1991

Mam, J., Catton, I., "One dimensional vs. Analysis into Normal Modes Solutions to Flow Induced Vibrations in a Square Cylinder Array", Proc. Ilth InL Conference on Struct. Mechanics in Reactor Technology, Vol. J, paper J08/2, Tokyo, Japan, 1991

Marn, J., Catton, I., "Flow Induced Vibrations of Cylindrical Structures Using Vorticity Transport Equation", Proceedings of the Winter Annual Meeting, Atlanta GA, 1991

Toyama, S., Mori, H., Suzuki, M., "Flow Patterns Through Tube Bundles and Particulate Fouling onto Cylinders", Particulate Science and Technology, pp. 397-408, No. 4, Vol 5, 1987

Sparrow, E.M., Molki, M., "Effect of a Missing Cylinder on Heat Transfer and Fluid Flow in an Array of Cylinders Cross-Flow", Int J. Heat Mass Transfer, pp. 449-456, No. 4, Vol 25, 1982

Leicher, M., Marn, J., and Catton, I., "Flow Visualization of a Square Array of Cylindrical Tubes: An Experimental Study Using Laser Doppler Anemometry", Proc. 1991 ASME Winter Annual Meeting, Vol. FED-128, pp. 217-224, Atlanta, GA, 1991

Andjelic, M., and Popp, K., 1989, "Stability effects in a normal triangular cylindrical array." Journal of Fluids and Structures, Vol.3, pp.165-185.

Lever, J.H., Weaver, D.S., " On the Stability Behaviour of Heat Exchanger Tube Bundles", Parts I and IL 1984 Symp. on Flow Induced Vibrations, Vol. 2, ASME

Dhaubhadel,M.N., Reddy, J.N., Telionis, D.P., "Finite-Element Analysis of Tubes is a Normal Triangular Array", Flow Induced Vibrations 1990, pp. 87-98, Vol. PVP-189, Nashville, TN, 1990

Taylor, CE., Pettigrew, M.J., Axisa, T., and Villard, B., "Experimental Determination of Single and Two-Phase Cross Flow-Induced Forces on Tube Rows," ASM FTV, PVP 104, pp. 31-39,1986

Blevins, R.D., Flow Induced Vibrations, Van Nostrand Reinhold, New York, 1990.

Chen, S.S., Jendrzejcyk, J.A., "Experiments on Fluid Elastic Instability of Tube Banks Subjected to Liquid Cross Flow", Journal of Sound and Vibration, Vol. 78, pp. 355-381, 1981 
Pettigrew, M.J., Tromp, LH., Taylor, C.E., Kim, B.S., "Vibration of Tube Bundles in Two-phase Cross-Flow: Part 2 - Fluidelastic Instability", 1988 Int. Symposium on Flow-Induced Vibration and Noise, 1988 ASUE Winter Annual Meeting, Chicago, Illinois, Vol.3

Pettigrew, M. J., Taylor, C. E., Kim, B. S., "The Effects of Bundle Geometry on Heat Exchanger Tube Vibration in Two-Phase Cross Flow," Journal of Pressure Vessel Technology, 123, pp. 414-420, 2001

Goyder, H.G.D., "Three methods for determining natural frequencies and damping ratios from vibration spectra." Proceedings of the Third International Conference on Recent Advances in Structural Dynamics. Air Force Wright Aeronaut. Labs. 1988, pp.279-88 vol.1. Wright-Patterson AFB, OH, USA

Franklin, R.E. and Soper, B.M.H., "An Investigation of Fluidelastic Instbilities in Tube Banks Subjected to Fluid Cross-Flow," Paper F6/7 in Proceedings of Conference on Structural Mechanics in Reactor Technology, San Francisco, CA, 1977

Southworth, P.J., Zdravkovich, M.M., "Effect of Grid Turbulence on the Fluid Elastic Vibrations of In-line Tube Banks in Cross-Flow," Journal of Sound and Vibration, Vol. 39, p. 461469,1975

Yetisir, M., Weaver, D.S., "On an Unsteady Theory for Fluidelastic Instability of Heat Exchanger Tube Arrays", 1988 Int. Symp. on Flow Induced Vibrations and Noise, Vol. 3, ASME

Catton, I., and Marn, J., "Some Models of the Prediction of Fluid Elastic Instability," Nucl. Engr. and Design, Vol. 139, No. 1, pp. 105-119

Mam, J., "Cross Flow induced Vibrations in Arrays of Cylindrical Structures", Ph.D. Dissertation, 1991

Feenstra, P. A., Weaver, D. S., and Nakamura, T., "Vortex Shedding and fluidelastic instability in a normal square tube array excited by two phase cross-flow" Journal of Fluids and Structures, 17, 793-811, 2003

Tanaka, H., Takahara, S., "Fluid elastic vibration of tube array in cross flow", Journal of sound and vibration Vol. 77 No 1, 1981, pp 19-3

Connors, H. J., Jr., "Fluidelastic Vibration of Tube Arrays Excited by Cross-Flow," In Flow Induced Vibration in Heat Exchangers, ASME Winter Annual Meeting, New York, 1970

Weaver, D. S., Ziada, S., Au-Yang, M. K., Chen, S. S., Paidoussis, M. P., Pettigrew, M. J., "Flow-Induced Vibrations in Power and Process Plant Components -Progress and Prospects," Journal of Pressure Vessel Technology, 122, pp. 339-348, 2000

Axisa, F., Villard, B., Gilbert, R.J., Hestroni, G., and Sundheimer, P., "Vibration of Tube Bundles Subjected to Air-Water and Steam-Water Cross Flow: Preliminary Results on Fluidelastic Instability," Flow-Induced Vibration, ASME Winter Annual Meeting: New Orleans, pp. 269-284, 1984 
Axisa, F., Villard, B., Gilbert, R.J., Boheas, M.A, "Vibration of Tube Bundles Subjected to Steam-Water Cross Flow: A Comparative Study of Square and Triangular Pitch Arrays," Transactions of the $8^{\text {th }}$ International Conference on Structural Mechanics in Reactor Technology, Vol. B., Brussels, pp. 7-12, 1985

Feenstra, P.A., Judd, R.L., and Weaver, D.S., "Fluidelastic Instability in a Tube Array subjected to Two-Phase R-11 Cross-Flow," Journal of Fluids and Structures, 9, pp. 747-771, 1995

Hirota, K., Nakamura, T., Kasahara, J., Mureithi, N. W., Kusakabe, T., and Takamatsu, H., "Dynamics of an In-Line Tube Array subjected to Steam-Water Cross-Flow. Part III: Fluidelastic Instability tests and Comparison with Theory," Journal of Fluids and Structures, 16(2), pp. 153-173, 2002

Joo, Y., and Dhir, V.K., "On the Mechanism of Fluidelastic Instability of a Tube Placed in an Array Subjected to Two-Phase Crossflow," Journal of Fluids Engineering, 117, pp. 706712,1995

Joo, Y., and Dhir, V.K., "An Experimental Study of Drag on a Single Tube and on a Tube in an Array under Two-Phase Cross Flow," International Journal of Multiphase Flow, 20, pp. 1009-1019, 1994

Pettigrew, M.J., Tromp, J.H., Taylor, C.E., Kim, B.S., "Vibration of Tube Bundles in Two-Phase Cross-Flow: Part 2 -Fluid-Elastic Instability," Journal of Pressure Vessel Technology, 111, pp. 478-487, 1989

Schröder, K., and Gelbe, H., "New Design Recommendations for Fluidelastic Instability in Heat Exchanger Tube Bundles," Journal of Fluids and Structures, 13, pp. 361-379, 1999 